\title{
THEORY OF SCHOTTKY BARRIER AND METALLIZATION
}

\author{
I.P. BATRA \\ IBM Research Division, Almaden Research Center, Mail Stop K62/282 \\ 650 Harry Road, San Jose, California 95120-6099, \\ and \\ E. TEKMAN and S. CIRACI \\ Department of Physics, Bilkent University, Bilkent 06533, Ankara, Turkey
}

\begin{abstract}
.
The formation of the rectifying Schottky barrier on metal-semiconductor interfaces is one of the longest standing problems of solid-state physics. We present a review of the models and theories for Schottky barrier. Two important examples of metal-semiconductor interfaces, namely those containing simple and alkali metals, are analyzed in order to evaluate these models and theories in the light of ab-initio calculations.
\end{abstract}

\section{Contents}

Page

1. Introduction

2. Theoretical Aspects

$\begin{array}{lll}\text { A. Definition of Schottky Barrier } & 293 \\ \text { B. Effects of the semiconductor surface states } & 295 \\ \text { C. Metal induced gap states } & 297 \\ \text { D. The index-of-interface } & 301 \\ \text { E. More MIGS models } & 304 \\ \text { F. The defect models } & 309 \\ \text { G. Adsorbate-induced states } & 314\end{array}$

3. Simple metal-semiconductor interface 316

A. Submonolayer coverage and overlayer metallization 317

B. Multilayer coverage and electronic structure 320

C. Fermi level pinning at different coverages 326 
4. Alkali metal-semiconductor interface

A. Work function $\quad 330$

B. Alkali coverage $\quad 330$

C. Adsorption site $\quad 332$

D. Structure at $\theta=1(1 / 2$ a ML coverage)

E. Peierls' distortions $\quad 337$

F. Fully optimized structure

G. Structure at $\theta=2$ coverage

H. Third order $(\theta=2 / 3)$ structure

I. Nature of binding and electronic structure 347

References

\section{Abbreviations}

1D one-dimensional

2D two-dimensional

AB Abraham-Batra

AES Auger electron spectroscopy

AM alkali metal

ARUPS angular resolved ultra-violet photoemission spectroscopy

AUDM advanced unified defect model

EELS electron energy-loss spectroscopy

INDO intermediate neglect of differential overlap

LDOS local density of states

LEED low-energy electron diffraction

MIGS metal induced gap states

ML monolayer

NEA negative electron affinity

SCF self-consistent field

SEXAFS surface extended x-ray absorption fine structure

STM scanning tunneling microscopy

UDM unified defect model

WKB Wentzel-Kramers-Brillouin 


\section{Introduction}

Metal-semiconductor (M-S) interfaces have been an active field of research for several decades in both fundamental and applied condensed matter physics. Interest was generally directed to the modifications of the electronic and atomic structure of semiconductor surfaces induced by the adsorption of metal atoms. Our understanding of the chemisorption process and surface reconstruction have advanced due to the efforts of a vast number of researchers in this field. In the same context, a great deal of effort has gone towards developing a microscopic understanding of interactions at the M-S junctions. Novel electronic properties of M-S interfaces have enormous commercial applications ranging from infrared detectors to very large scale integrated circuits.

When a semiconductor is put in an intimate contact with a metal, its bands become aligned so that they establish a common chemical potential. This usually leads to a shift of the semiconductor bands. Since the states with energies within the energy gap of the semiconductor must decay into the semiconductor, the upward shift of the conduction band edge and the resulting band bending (in the case of an n-type semiconductor) forms a barrier, which goes by the name Schottky barrier[1] (SB), for electrons incident from the metal. Hence, metal electrons with energies falling in the gap are prevented from entering into the semiconductor. Upon application of an external voltage to the junction, the energy difference between the semiconductor quasi Fermi level and the top of the barrier is changed. Under forward bias conditions, the thermal population of majority carriers which can be transmitted over the top of the barrier is enhanced, and thus a current flows. However, no such effect occurs when a reverse bias is applied. The ability to force the current to flow in one direction and not in the reverse direction leads to junctions which are rectifying in nature. Owing to its rectifying properties $\mathrm{SB}$ is an essential ingredient of electronic devices[2].

The Schottky model was designated originally for calculating the potential barrier for M-S rectifiers. It assumed a depletion layer (a region devoid of mobile carriers) on the semiconductor side containing a constant density of (uncompensated) ionized donor impurities for an n-type doped semiconductor with a work function less than the work function of the metal. Then from Poisson's equation it immediately follows that the electrostatic potential must vary quadratically with distance as the metal is approached from the semiconductor side. The barrier height then simply is equal to the difference between the metal work function and the electron affinity of 


\section{I.P. Batra et al.}

the semiconductor. If the values of the work functions are reversed (a low work function metal in contact with a high work function semiconductor) the resulting junction leads to Ohmic properties.

In the past, a number of scientists who were challenged by the intriguing patterns of the measured SB heights, attempted to develop a universal theory. Unfortunately, these efforts have not achieved their ultimate objective because of many complicating factors which determine the transfer of charge at the interface. The experimental data accumulated so far indicate that several factors (such as coverage, interface structure, growth conditions, defects, etc.) can influence the formation of SB. The important but not fully understood issue is why the Fermi level gets pinned. In particular, the precise nature and origin of the electronic states responsible for the pinning have been the subject of much controversy.

The field of Schottky junctions and the barrier derived thereof has been the subject of several review articles[3-5]. Therefore, in the present exposition we keep the scope of our work rather focused and mainly concentrate on some of the more recent developments. In section 2 , we give a background of some theoretical aspects of the M-S interfaces with an emphasis on the earlier models proposed for the formation of SB. We start with the historical definition of Schottky barrier and summarize major theories essentially in the chronological order. These are Bardeen's theory[6] on the Fermi level pinning, metal induced gap states[7], charge neutrality level[8,9], defect models[1012], adsorbate induced interface states[13-15], etc. This sets the stage for discussion in further sections. Section 3 examines results of first principle calculations obtained for a lattice matched M-S interface. Owing to the perfect lattice match some factors, which would otherwise contribute to the pinning of the Fermi level, are eliminated. This allows one to concentrate only on the gap states which decay on one or both sides of the junction. The specific system we consider is the Al-Ge(001) interface and we use a wide range of metal coverages. An extensive discussion on the role of specific states (such as metal induced gap states, interface states and surface resonances) in determining the Fermi level is presented. An important point, which has not received an adequate attention, is how the metallization of the absorbed overlayer sets in. We discuss the overlayer metallization based on earlier investigations on this subject.

For those semiconductors which have active surface states near the Fermi level, the metallization of the overlayer must compete with the formation of M-S bonds. In fact, depending on the relative value of the metallic cohesive energy and the metal-substrate interaction energy, the overlayer metallization may be suppressed altogether. In this respect, the adsorption of alkali metal atoms on other metal and semiconductor surfaces is special, and presents fascinating ideas about bonding, metallization, Fermi level pinning, stability, and collective excitations as a function of coverage. 
The recent controversies about the nature of bonding and metallization of alkali metal overlayer on Si surfaces have motivated research which has shed light on some important features of the problem. The last section of our review is devoted to this vivid subject. In particular, we discuss different views on the overlayer metallization and present results of recent theoretical works on the stability of overlayer against Peierls' type of distortion and energetics of adsorption structure. As far as the alkali metal absorption on the $\mathrm{Si}(001)-(2 \times 1)$ surface is concerned the present article is the only one which reviews this subject in depth.

\section{Theoretical Aspects}

\section{A. Definition of Schottky barrier}

The problem of SB formation has been treated by several theoretical methods, ranging from classical electrostatics to many-body theory, and from one-dimensional tight-binding models to self-consistent field local-density approximation with realistic structures. The height of the SB for semiinfinite metal-semiconductor systems was the subject matter of the pioneering studies. With the advent of more sophisticated experimental methods, attention has turned to monolayer metal coverage regime and to the microscopic aspects of the metal-semiconductor interactions.

The first explanation of the rectifying barrier at a metal-semiconductor contact was provided by Schottky[1]. He argued that while isolated semiinfinite metal and semiconductor are separately in thermal equilibrium (and thus each has its own Fermi level established as a function of density of electrons, temperature, etc.), their thermal equilibrium will be altered when the two bodies are put in intimate contact as shown in Fig. 1. To recover thermal equilibrium the Fermi level has to be the same across both materials. This can be achieved by forming a dipole moment as a result of charge transfer from the semiconductor into metal conduction band (or reverse). This creates a depletion region of finite extent made by the ionized dopants (that is, free of mobile charge carriers). We will refei to this explanation as the Schottky model hereafter. The height of the barrier can be deduced by simply using the bulk properties of the constituents. Namely the electron affinity $\chi_{s c}$ of the semiconductor and the metal work function $\phi_{m}$. The SB height is given as

$$
\phi_{B n}=\chi_{s c}-\phi_{m}
$$

In what follows we denote the SB height as $\phi_{B d}$ where $d$ is the type of doping ( $n$ or $\left.p\right)$. $\phi_{B n}\left(\phi_{B p}\right)$ 
is the energy difference between the conduction band minimum (valence band maximum) of the semiconductor and the Fermi level at the interface. That is, $\phi_{B d}$ is the height of the potential barrier for charge carriers passing from the metal to semiconductor. Note that $\phi_{B n}$ and $\phi_{B p}$ add up to the energy gap of the semiconductor, $E_{g}$.

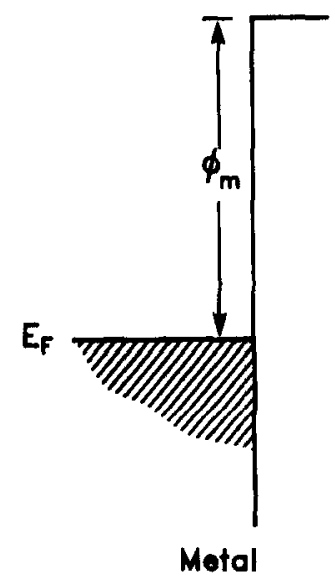

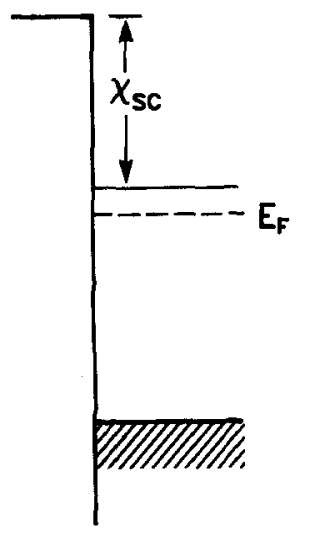

Semiconductor

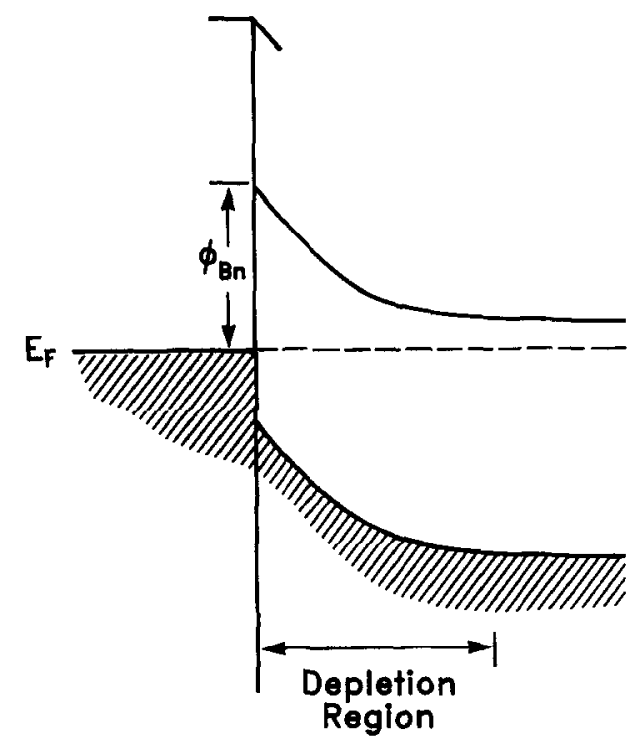

(b)

Fig. 1. Energy band diagrams of a metal and an n-type semiconductor (a) for infinite separation and (b) intimate contact.

Due to its lucidness the Schottky model has been extensively used in most of the practical considerations. However, detailed experiments showed that it can not fully explain the phenomena taking place at a M-S interface. This has led to search for new models which are described in detail below. However, eventually it was appreciated that the Schottky-like behavior is one of the extreme cases for a rectifying barrier, and for certain systems it is experimentally achievable as well. One of the main observations on the SB height is that it is due to two types of dipoles formed at or around the interface, which have different origins. One of these is the dipole due to the space charge in the depletion layer. That is, the one that was considered by Schottky. A second dipole may arise at the interface which is confined to a few atomic layers, and may have significant effect on the SB height. The latter effect was completely ignored by Schottky. Recently Mailhiot and 
Duke[16] showed that the Schottky model, in fact, may incorporate such a microscopic dipole as well. They modeled the metal and semiconductor using a local-density functional for the ground state energy in the jellium approximation. Imposing thermal, mechanical, and electron-transfer equilibrium they calculated the self-consistent charge density and potential for the combined M-S system. The resulting potential arose from a space charge contribution and a microscopic interface dipole. They found that the microscopic dipole has a negligibly small contribution for typical dopant concentrations. Thus, the SB height determined from the boundary conditions deep in the metal and semiconductor, as proposed by Schottky, is consistent with a many-electron minimum energy configuration. Note that, the use of the jellium model is an essential assumption of this model since it neglects all interactions due to discrete atomic structure. The microscopic dipole contribution arises as a result of the variation of electron density across the interface. These variations are small when calculated in the jellium approximation and hence one gets only a minor contribution from the microscopic dipole.

Later a variety of experimental results suggested that the chemical reactions between the metal and semiconductor atoms at the interface have important effects on the SB height. Freeouf and Woodall[17] reexamined the Schottky model in the light of these results. They suggested that due to chemical reactions the semiconductor is not in contact with the original metal with work function $\phi_{m}$. That is, the species formed as a result of the chemical reactions form a mixture of microclusters of different phases. Therefore, it is necessary to average out the work functions over these microclusters. The SB height is then determined by an effective work function $\phi_{m}^{\mathrm{eff}}$, and not the metal work function $\phi_{m}$,

$$
\phi_{B n}=\chi_{s c}-\phi_{m}^{\text {eff }}
$$

For most of the III-V and II-VI compounds it was argued that the chemical reactions either free anions or form metal-anion complexes. This leads to a common anion rule, for which $\phi_{m}^{\text {eff }} \simeq \phi_{\text {anion }}$. The deviations from the common anion rule were attributed to specific reactions which do not let anion species to form.

\section{B. Effects of the semiconductor surface states}

The simple interpretation for SB in terms of electron affinity difference failed in some experimental tests made just a few years after it was first proposed. For a number of semiconductors it was found that the height of the rectifying barrier did not vary with deposited metal type. That is, the Fermi level was pinned at a certain position in the energy gap. These results suggested that 
the SB has an important contribution from the microscopic interface dipole, in addition to the conventional depletion dipole. Bardeen[6] proposed that the surface states of the semiconductor are responsible for the pinning of the Fermi level. The most important suggestion of Bardeen was the possibility of acquiring a surface state charge which leads to formation of a dipole layer confined to the interface. The barrier height is determined by this dipole layer and the space charge in the semiconductor, thus it differs from the Schottky term given by Eq. (1).

At that time the surface states were not well understood, so Bardeen used a simple model to incorporate the effects of the surface states on the SB height. He assumed that the density of surface states is constant in the energy gap of the semiconductor, and the total surface state charge is zero when the Fermi level lies at $E_{s s}$ (the energies are defined with respect to the valence band maximum of the semiconductor). Thus, the density of surface state charge $\sigma_{s s}$ is a constant times $\left(E_{F}-E_{s s}\right)$. The density of space charge $\sigma_{s c}$ varies with square root of the band bending as found by Schottky. The total dipole which counterbalances the electron affinity difference at the interface, in turn, is formed by the total charge transfer to the metal $\sigma_{s s}+\sigma_{s c}$. Bardeen assumed that a gap of width $w$ is present between the metal and semiconductor. Accordingly as $w$ decreases, both the charge required for offsetting the electron affinity difference increases and the density of surface states decreases due to the bonding to the metal. However, in the 1940's the rectifying metal-semiconductor junctions were obtained by pressing metal and semiconductor surfaces both of which have oxide layers on top, thus a fraction of the surface states would be active even when $w$ is on the atomic scale. T'he minimum number of surface states necessary to form an appreciable contribution to the SB height was found to be only a few percent of the number of surface atoms $\left(\sim 10^{13} \mathrm{~cm}^{-2}\right.$ ). From this point of view, Bardeen's explanation was very appealing since even with the best fabrication techniques at that time this condition was satisfied.

The barrier height can be found easily for the two extreme cases. If the density of surface states is very small, $\sigma_{s s}$ may be neglected and the charge in the semiconductor is solely due to depletion. This leads directly to the Schottky model where the barrier height is determined entirely by the electron affinities of the constituents, as given in Eq. (1). On the other hand if the density of surface states is very large, one can neglect the space charge. That is, all the transferred charge resides in the surface states. Since the density of surface states is very large only a minor difference between $E_{F}$ and $E_{s s}$ will create the necessary charge transfer. Thus, the barrier is determined by the charge neutrality level of the semiconductor in the absence of the metal. Then the SB height is given as

$$
\phi_{B n}=E_{g}-E_{s s}
$$


This complete pinning by surface states is called the Bardeen model. The Bardeen model found wide support from the experimental observations. In spite of the fact that this explanation was originated from purely intuitive grounds, it contains several conceptually important points which later were shown to be the benchmarks for a complete theory of SB formation. However, with advances in vacuum techniques and also in the fabrication of M-S junctions, it was found that some of the new data was at variance with the predictions of the Bardeen model. On the grounds that the surface states are eliminated upon the chemisorption of metal atoms or the reconstruction of the semiconductor surface, Bardeen's model was thought to be incomplete. Recently Lefebvre et al.[18] suggested that Bardeen's explanation agrees with the experiments if the broadening of the surface states due to interactions with metals is taken into account. In this context the surface states become resonances and the dipole layer required for the Fermi level pinning is obtained by partial filling of these resonances.

\section{Metal induced gap states}

Heine[7] proposed a new model which later developed into a Metal Induced Gap States (MIGS) model for metal-semiconductor interfaces. He allowed for the possibility that the surface states are not necessarily present on all cleaved semiconductor surfaces. Nevertheless, he stressed that the solutions of the Schrödinger equation for the M-S system include states propagating in the metal side but decaying into the semiconductor. These are similar to the surface states of the semiconductor. In this respect Heine extended the Bardeen model, which solely relies on the surface states. The main ingredient is that the metal electronic structure is taken into account in addition to the semiconductor band structure.

In his original work Heine[7] solved an effective one-dimensional problem which was later extended to real three-dimensional systems by several groups. In a nearly-free electron approximation the semiconductor and metal have Fermi surfaces which are slightly distorted spheres. Since the valence electron density of the semiconductors (four electrons per atom for $\mathrm{Si}$ ) is large compared to that of a metal, the radius of the Fermi sphere of the semiconductor is larger than that of the metal. Assuming specular transmission through the interface (where parallel momentum is conserved) Heine found that some of the semiconductor valence band states decay into the metal. The surface states at the zone edges with large parallel momenta have small normal wave vectors, and thus they are likely to penetrate into the metal. The resulting coupling (which is small due to its decaying nature) between the semiconductor and metal leads 
to formation of a Breit-Wigner resonance at the surface state energies. The resonance states form for metals with small electron densities, such as alkali and noble metals. In the opposite case of simple metals, the densities in the metal and semiconductor are comparable. For these interfaces the motal conduction band states lying in the semiconductor band gap have charge densities spilling into the semiconductor. These states were later named as MIGS. The integrated density of states of MIGS has its maximum at the position of the semiconductor surface state as the Breit-Wigner resonances have. These results shown in Fig. 2 clarified the effect of the surface states on the electronic structure of the interface. Heine also argued that the boundary conditions at the interface would have little effect on the density of states in the semiconductor band gap. On these grounds he justified the pinning position of the Fermi level to be independent of the metal work function, semiconductor doping, orientation of the surface, bias voltage and interface quality. This explanation of SB formation is referred to at the Heine or MIGS model.

This attempt to explain the SB by Heine is a composite of a Bardeen-like pinning effect and a Schottky-like space charge effect. Later it was experimentally shown that depending on the semiconductor and metal it is possible to be in either of these limits. It is fair to say that the origin of the modern theories of SB formation lies in Heine's work. Results of extensive $a b$-initio calculations confirm Heine's proposal. In sections 3 and 4 we discuss results for MIGS at the (Al) simple metal Ge interfaces and surface state resonances at (K) alkali metal-Si interface based on the ab-initio calculations. Furthermore, we clarify the relation between MIGS and possible resonance states developed from semiconductor surface states.

The formation of $\mathrm{SB}$ in the Heine model was influenced by the microucopic interface dipole as in the Bardeen model. However, the mechanism for the transfer of electrons was not like those proposed by Schottky or Bardeen. As pointed out above, MIGS have tails decaying into the semiconductor. Thus they have certain charge density in the semiconductor side in spite of the fact that they are metallike states. This transferred charge density forms the corresponding microscopic dipole. The dipole was proportional to the charge density per unit area $\sigma$ and the mean distance between the charges in the semiconductor due to the tails of MIGS and the opposite screening charges in the metal. This distance is determined by the screening length of the metal $\lambda_{\text {scr }}$ and the mean separation between the charges due to MIGS on the semiconductor side and the interface plane, $t$, screened by dielectric constant of the semiconductor $\epsilon_{s c}\left(i, e ., t / \epsilon_{s c}\right)$. Even though the first term (the screening length) was taken to be constant for different metals, special emphasis was given to the latter. To good approximation the charge density in the semiconductor falls exponentially with the decay constant $q$ limited by the complex band structure of the semiconductor 


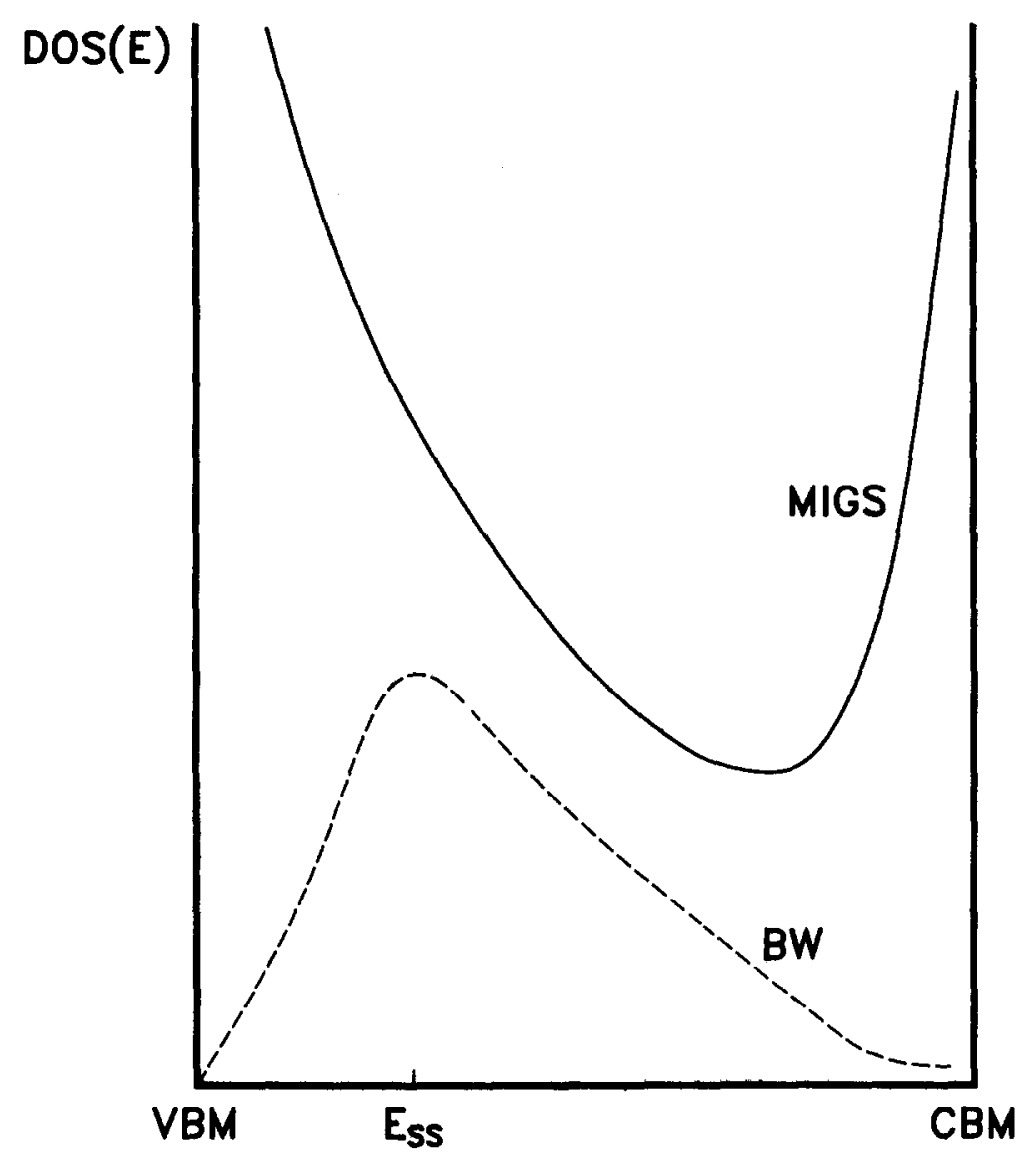

Fig. 2. Density of states of the metal induced-states for the metal-semiconductor interface. Full (dash-dotted) curve corresponds to the metal-induced gap states (Breit-Wigner resonances) and typical for high (low) electron density metals. CBM and VBM denote the conduction band minimum and valence band maximum, respectively. $E_{s s}$ denotes the semiconductor surface energy.

so that $q \leq q_{\max } \simeq 2 m E_{g} / \hbar^{2} k_{g}$, where $E_{g}$ and $k_{g}$ are the band gap and the wave vector for the conduction band minimum of the semiconductor (assuming that the band gap is indirect as it is for $\mathrm{Si}$ ), respectively. The charge density behaves like $\exp (-2 q z)$ in the semiconductor and the center of gravity of the electrons is $1 /(2 q)$ away from the interface. The typical values for silicon yields $t \geq 6 \AA$ which is covering only a few layers of the semiconductor. Heine deduced using the work function of silicon as a function of cesium coverage that $t \simeq 8 \AA$ which was consistent with both the theoretical estimate given above and the independence of the SB height from the bias 


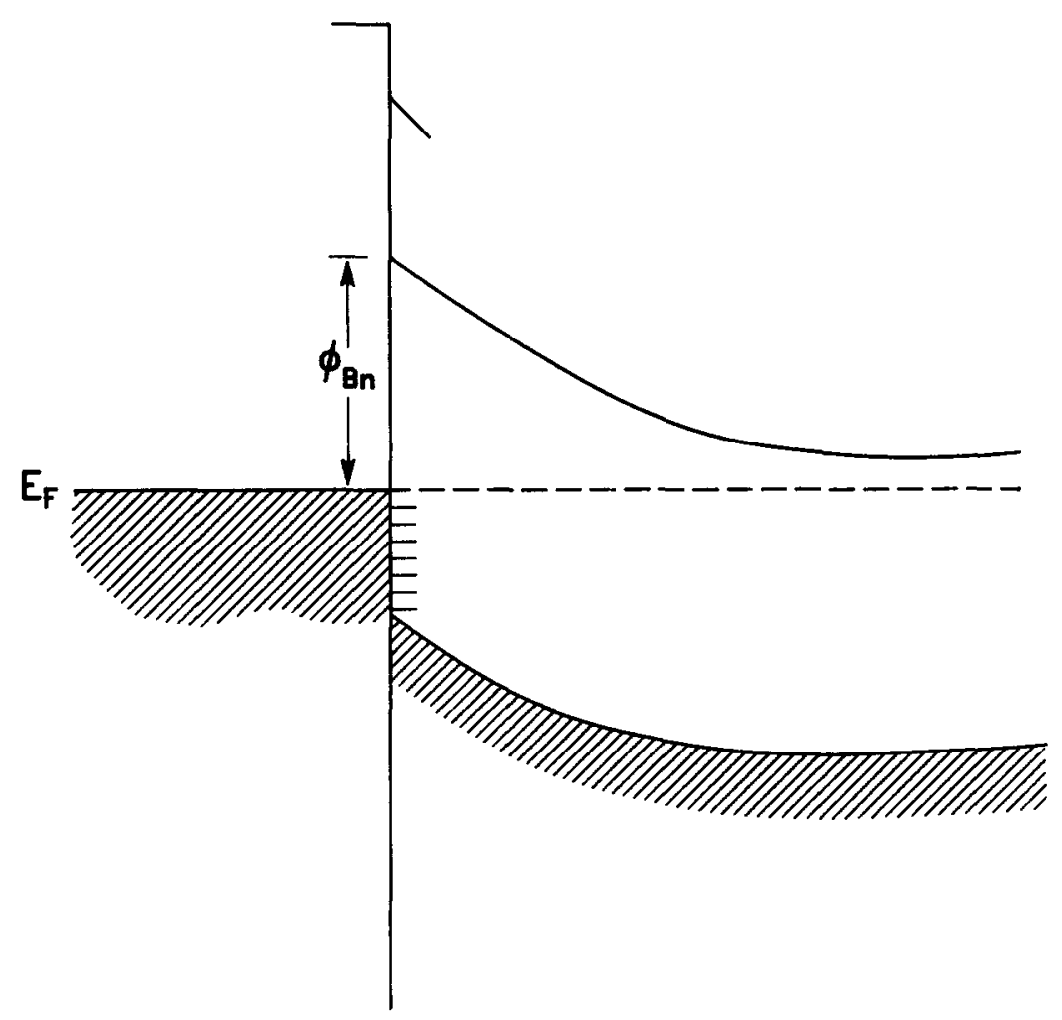

Fig. 3. Energy band diagram of the metal-semiconductor interface in the presence of metal-induced or defect states. Note that the band bending due to depletion is smaller than that in Fig. 1.

voltage. To this end, the effective (including the dielectric screening in the semiconductor) mean separation for the dipole was estimated to be $1.2 \AA$.

In order to achieve thermodynamic equilibrium, the total dipole has to exactly align the Fermi levels in the metal and semiconductor. Due to the above mentioned microscopic dipole the Fermi level at the vicinity of the interface rises with respect to the valence band maximum (or equivalently the semiconductor bands bend down). Therefore the SB height deviates from the value given by the Schottky model, $\chi_{s c}-\phi_{m}$. This effect of the microscopic dipole is shown schematically in Fig. 3. To get quantitative results Heine assumed that prior to the metal deposition the Fermi level was located at the surface state energy $E_{s s}$ and the MIGS have a constant density of states $N\left(E_{F}\right)$ at energies close to $E_{F}$ and $E_{s s}$. By using uniform distribution of surface states in the gap, Heine gave a lower limit for $N(E) \sim 10^{14} \mathrm{~cm}^{-2} \mathrm{eV}^{-1}$ which was still larger than the minimum 
density of states required to pin the Fermi level. Finally the SB height was found to be given by

$$
\phi_{B n}=E_{g}-E_{s s}+A\left(\chi_{s c}-\phi_{m}\right),
$$

the coefficient of the Schottky term being $A=0.13$ for Si which was in agreement with the general trend of the experimental data.

\section{The index-of-interface}

At this point it is in order to mention the transition taking place between the systems obeying the Schottky model and those obeying the Bardeen model. Bardeen[6] and Heine[7] successfully showed that although the Fermi level is likely to be pinned by states in the semiconductor band gap, the Schottky term has also an effect on the SB height. A measure for this tendency is given by the index of the interface behavior, $S$, which is defined as

$$
S=\frac{d \phi_{B n}}{d \phi_{m}}
$$

Clearly $S$ changes between 0 (the complete pinning case) and 1 (the ideal Schottky case). As early as 1965 Cowley and Sze[19] argued that the index-of-interface may be related to the density of interface states. However, at that time a quantitative study on the interface states was not available and thus they could not support their model with accurate results. Later, interpreting a large number of compiled results Kurtin et al.[20] suggested that it is possible to assign an $S$ value to the semiconductor, independent of the metal used. They found that for covalent semiconductors $S \simeq 0$ and for highly ionic ones $S \simeq 1$. Thus, they classified the semiconductors into two broad groups (ionic and covalent) according to binding properties, which determine their behavior at metal interfaces. Plotting $S$ as a function of the electronegativity difference $\Delta X$ of the anion and cation of the semiconductor they discovered that there is an abrupt transition between the covalent and ionic semiconductors around $\Delta X \simeq 0.7$. This observation was supported by similar behaviors such as the relative contribution of nondirect transitions in photoelectron studies and the relative strength of exciton absorption. They attributed this transition to many-electron effects which become very important for ionic semiconductors and argued that a perturbative treatment can not reconcile this transition.

Later the problem of covalent-to-ionic transition was attacked by methods involving manyelectron interactions as well. Phillips[21] discussed the effects of charge rearrangement at the interface in terms of the total energy of the systems. He pointed out that the creation of a dipole at the interface increases the long-range contribution to the surface energy which is proportional to 
the zero point energy of the interface plasmons. On the other hand the charge transfer excitations give rise to an energy gain which is proportional to the average optical gap of the semiconductor. In order to have a dipole layer at the interface, which pins the Fermi level, these two energies have to be comparable. Relating the semiconductor dielectric polarizability to these two parameters Phillips concluded that the covalent-to-ionic transition is a function of the dielectric constant of the semiconductor $\epsilon_{s c}$. For weakly-ionic and covalent semiconductors the average band gap is large enough to compensate for the long-range energy gain due to charge transfer and the Fermi level is pinned by the dipole layer. On the other hand, for highly ionic semiconductors the plasmon energy is so large that the charge transfer is prohibited leading to a Schottky-like behavior.

Inkson[22] analyzed the effects of screening at the vicinity of the interface by using a manyelectron approach. He found that the screening due to the correlation effects can be attributed to exchange of plasmons and bends the conduction and valence bands near the interface downwards. This is the completion of the incomplete semiconductor screening due to the energy gap. On the other hand, screened exchange interaction has a large anisotropy as far as the conduction and valence bands are concerned. This leads to an upward bending of the valence band which is twice as large as the correlation effect. Consequently, the energy gap of the semiconductor shrinks near the interface. For covalent semiconductors this effect is very strong, leading to merge of the two bands and the pinning of the Fermi level in this continuum of states. However, for ionic semiconductors the effect is not as dramatic, leading to the Schottky-like behavior of the interface.

Later the covalent-to-ionic transition was attributed to the chemical reactivity of the metals[23]. According to these models, the chemical species formed at the interface cause a microscopic rearrangement of charge which pins the Fermi level to some extent depending on the semiconductor and metal. The index-of-interface $S$ was shown to have a transition behavior as a function of the heat of formation $\Delta H_{f}$ for the product of the chemical reaction. However, these empirical explanations have not been verified by quantitative studies so far. On the other hand, Schlüter[24] objected to the Schottky limit $(S=1)$ proposed by the others, based on the difference between the theoretically defined electronegativities and the experimentally obtained ones. He suggested that the saturation occurs for $S \simeq 2.3$ and the transition is not as sharp as thought to be. Using Heine's approach[7] he derived an empirical relation between the total polarizability of the semiconductor, $\epsilon_{s c}$ and the $S$ value. Recently Mönch[25] reevaluated the pioneering study of Cowley and Sze[19] using a single oscillator model. He showed that the dipole contribution from the interface states is related to the electronic part of the semiconductor dielectric function $\epsilon_{\infty}$.

At this point we discuss the interesting work by Mele and Joannopoulos[26] on the covalent-to- 
ionic transition. They used the energy difference $\Delta E_{s p}$ between atomic s-level of the cation and p-level of the anion of the semiconductor as the measure of ionicity. They justified the relation between $\Delta E_{s p}$ and ionicity by using the tight-binding model for the band structure. Namely, for negative $\Delta E_{\text {op }}$ the energy gap depends strongly on the hybridization of $s$ - and p-levels (covalent semiconductors), on the other hand for positive $\Delta E_{\text {op }}$ the gap is present even when the hybridization is absent (ionic semiconductors). They analyzed the interface behavior of the semiconductors by employing the Heine model[7]. They found transitive behavior for both the energies and lifetimes of the MIGS in a SB, which is attributed to covalent-to-ionic transition. For covalent semiconductors the integrated density of MIGS have a main peak near the center of the energy gap and thus interact strongly with the metal wave functions. This leads to pinning of the Fermi level, thus to a small $S$ value. However, for ionic semiconductors the surface states are near the band edges and are not hybridized. Consequently, they do not interact with the metal conduction band states. Therefore, they are inefficient in forming an interface dipole layer, leading to larger values of $S$. This model not only agreed with the experimental results but also explained the covalency of large gap materials as $\mathrm{C}$ and $\mathrm{SiC}$ which have negative $\Delta E_{\text {sp }}$ values.

Recently Chang et al.[27] made systematic analysis of the SB formed by depositing semiconductor on metal, that is by reversing the conventional growth sequence. They found that, the morphology of the interface is completely different than that obtained by depositing metal on the semiconductor. However, the interface chemical species and SB height was found to be independent of the deposition scheme. Based on these results they concluded that the index-of-interface could not be related to the dielectric constant or local density of states which highly depend on the morphology. They suggested that the interface dipole is locally determined by the electron affinity difference of the chemical species. Thus they supported the effective work function model[17] stressing that the pinning of the Fermi level by the interface states has to be taken into account with this simple explanation.

Brillson and Chiaradia[28] analyzed a large compilation of data and concluded that the Schottky model[1] in its original form is capable of explaining the results, while the other theories fail to give a complete picture to be applied to all of the cases. They attributed the discrepancies with the existing experiments which invalidate the Schottky model to nonideal interfaces and effects arising therefrom. 


\section{E. More MIGS models}

The Heine model[7] in its simple form was applied to real three-dimensional systems by Louis et al. [8]. Using an empirical pseudopotential method they calculated the SB heights for several zinc-blende semiconductors. They confirmed the presence of both Breit-Wigner resonances and virtual states (MIGS) in the semiconductor band gap as predicted by Heinc. They argued that the surface states of the semiconductor have significant effect on SB formation as pointed out by Bardeen[6] and Heine[7]. They also calculated the index-of-interface $S$ according to the expression proposed by Cowley and Sze[19]. Although their results for weakly-ionic cases were in agreement with experiment, they failed to explain the covalent-to-ionic transition in terms of this one-electron picture.

One of the important confirmations of the MIGS model came from the work of Louie and Cohen[29]. The objective of their study was the self-consistent analysis of the M-S interface by using band structure methods. Until then the explanations suggested for SB formation had been at the qualitative level out of which only some empirical relations were obtained. The developments in computational physics made it possible to calculate most of the bulk and surface properties of solids with reasonable accuracy. Therefore, in the late 1970's it was possible to simulate Schottky diodes in more realistic calculations.

Louie and Cohen[29] used a supercell consisting of a slab of $\mathrm{Si}$ in contact with a jellium representing Al. They used the self-consistent pseudopotential method with local exchange to find the energy bands, local density of states and self-consistent potential. They obtained the MIGS and interface states (Breit-Wigner resonances) conjectured by Heine[7] indicating that simple wave function matching arguments are approximately valid for the realistic systems as well. The charge transfer from the metal to the semiconductor was justified by use of the integrated charge densities, thus the interface dipole predicted by Bardeen[6] and Heine[7] was confirmed. They also showed that deep in the jellium the local density of states resembles to that of a free electron metal and in the semiconductor side it reproduces the density of states of the bulk Si. Near the interface, however, the local density of states was performing a transition from the semiconductor-like to metallic character. The important point was the nonzero density of states in the thermal energy gap of the semiconductor due to the MIGS. Consequently the Fermi level was pinned by these states in the Si band gap. Analyzing the charge densities of these gap states they found them to be similar to those predicted by Heine[7]. That is, they are propagating in the metal and decaying into the semiconductor in a few layers from the interface. Thus, the MIGS model of SB 
formation was shown to be consistent with realistic solutions of defect-free interfaces. Louie and Cohen[29] argued that the shrinkage of the band gap suggested by Inkson[22] does not take place for $\mathrm{Si}$. However, this point needs further investigation since the local exchange potential used by Louie and Cohen may be inappropriate for investigating subtle many-body effects pointed out by Inkson. Later, covalent-to-ionic transition was investigated by Louie et al.[30] using the above described supercell method. The index-of-interface was calculated according to the expression given by Cowley and Sze[19]. As a result of both the reduced density of states and smaller decay length of the MIGS, the highly ionic semiconductors lead to higher $S$ values as compared to the covalent ones. However, the abrupt transition observed by Kurtin et al.[20] was not completely confirmed due to the small number of systems studied.

The first theoretical elaboration of the Heine model was done by Tejedor et al.[31]. They aimed at finding the solution of the Schrödinger equation for the combined metal-semiconductor system with the inclusion of many-body interactions. The main achievement of this study was the generalization of Heine's proposition for $\mathrm{Si}$ to cover a wide range of elemental and compound semiconductors. The introduction of the concept of the charge neutrality level was one of the major outcomes of this model, since at that time the problem of surface states were resolved and it was known that the surface states do not define a specific energy in the band gap of the semiconductor.

For determining the SB height Tejedor and coworkers[31] identified the contributions to the dipole layer forming the barrier. In addition to the dipole created by difference of the semiconductor electron affinity and metal work function [the Schottky term given by Eq. (1)], they found that the crystal structures and different electron densities in the metal and semiconductor affect the barrier and give rise to corrections, denoted as $\Delta \chi_{J}$. They considered also the dipole created by the MIGS as Heine did, in order to equate the Fermi levels in the metal and semiconductor sides.

The equilibration of the Fermi level was described in conjunction with charge neutrality level. Note that, the contact between the metal and semiconductor does not affect the total number of electronic states, but merely rearranges their energies. Namely, some of the valence band states of the semiconductor couple to the metal states to form the MIGS. The charge neutrality level $\phi_{0}$ was defined as an energy below which the integrated density of MIGS is equal to the number of missing states in the semiconductor valence band. That is, in order to fill the states up to $\phi_{0}$ it is not necessary to transfer charge from one side to the other. The cancellation of the filled MIGS and missing valence band states of the semiconductor at the interface is determined by the microscopic properties of the interface and thus the charge neutrality level is defined locally. The charge neutrality level used by Bardeen[6] [which was denoted by $E_{s s}$ in Eq. (3)] was the global 
one, which is found by considering a neutral semiconductor surface, thus conceptually differs from the one defined by Tejedor et al.[31].

Using a WKB model for the metal and an empirical pseudopotential model for the semiconductor Tejedor and coworkers[31] calculated the density of states for the combined metal-semiconductor system. They found that below the top of the semiconductor valence band the density of states is given as the sum of the density of states of the metal and semiconductor sides as if they are completely decoupled. In the semiconductor band gap the density of states is the sum of the density of states of the metal and the density of MIGS. This density can be calculated using the phase shift of the wave functions matched at the interface. The charge neutrality level $\phi_{0}$ is found to be at the midgap energy for the simple model outlined above, which has a surface state at the midgap for the semiconductor surface, and the density of MIGS is symmetric along the gap.

In order to calculate the induced dipole at the interface Tejedor et al.[31] assumed that the charge lying in the states below $\phi_{0}$ behaves very much like a metallic charge density, thus the dipole due to the interface charges is calculated in the jellium approximation. To this end, they incorporated the screening due to polarization of the semiconductor. For the charge density of MIGS they used an analytical expression consisting of the long-range charge screened by the semiconductor and the charge piled at the interface which can not be screened due to its localized nature. The separation of charge between these terms was determined using a variational procedure to minimize the total electronic energy.

The surface barrier was calculated by adding the contributions from the metallic charge, MIGS and difference of the electron affinities. The resulting Schottky-like term is given by Eq. (1) where $\phi_{m}$ and $\chi_{s c}$ are the values associated with the crystallographic faces and the correction $\Delta \chi_{J}$ is added. Note that the dipole due to MIGS depends on the total number of MIGS. Since this density of states is averaged over the Brillouin zone of the semiconductor it is almost independent of energy around $\phi_{0}$. Therefore, it was taken to be a constant $N_{\text {MIGs and the associated dipole }}$ $D_{\text {MIGs }}$ found to be proportional to the deviation of the Fermi level from the charge neutrality level. Solving the resulting two equations simultaneously the position of the Fermi level was obtained. The final expression for the SB height was

$$
\phi_{B n}=\frac{1}{1+\alpha N_{\text {MIGS }}}\left[\left(\phi_{m}-\chi_{s c}+\Delta \chi_{J}\right)+\alpha N_{\text {MIGS }}\left(E_{g}-\phi_{o}\right)\right]
$$

$\alpha$ being a function of the electron densities of the metal and semiconductor. One point emphasized was that the SB height does not vary linearly with $\phi_{m}$ since $\alpha, N_{\text {MIGS }}$ and $\Delta \chi_{\mathrm{J}}$ are all functions of the metal. Therefore, the index-of-interface $S$ is not simply equal to $\left(1+\alpha N_{\text {MIGs }}\right)^{-1}$ but the 
average slope of the $\phi_{B n}$ versus $\phi_{m}$ curve. Also the two classical limits were obtained for extreme values of $N_{\text {MIGS }}$ as shown by Bardeen[6]. For a high density of metal induced interface states, i. e., $N_{\text {MIGS }} \rightarrow \infty$, the semiconductor forces the system to achieve local charge neutrality and the SB height is given by

$$
\phi_{B n}=E_{g}-\phi_{o}
$$

which is the result for the Bardeen model, since for the simple system studied there is a surface state just at the charge neutrality level. On the other hand for a system devoid of metal induced interface states, i. e., $N_{\text {MIGS }}=0$, the global thermodynamic equilibrium condition determines the position of the Fermi level

$$
\phi_{B_{n}}=\phi_{m}-\chi_{s c}+\Delta \chi_{J}
$$

which is the result of the Schottky model corrected by $\Delta \chi_{J}$.

In spite of several approximations involved, the method proposed by Tejedor et al.[31] is one of the key contributions to the MIGS model for SB formation. The main point of this study was to incorporate the effects due to charge transfer between the metal and semiconductor in a total energy minimization scheme in the jellium approximation. They found that local charge neutrality forces the Fermi level to be pinned at the charge neutrality level. On the other hand, the global thermodynamic equilibrium pushes the barrier height towards the Schottky term given by Eq. (1). The SB is formed by a balance between these two opposing effects. In contrast to its appealing form, the model could yield correct values of $\phi_{B_{n}}$ only for Al-Si and Na-Si interfaces and acceptable values of $S$ only for weakly-ionic zinc-blende semiconductors. The covalent-to-ionic transition was not explained by this model since $\alpha N_{\text {MIGS }}$ does not change much between covalentand ionic-semiconductors. This discrepancy between the theory and experiment was attributed to the inappropriateness of the use of one-electron techniques.

Later Flores and coworkers[32] proposed a self-consistent tight-binding method to analyze the SB height quantitatively. They assumed that the charge transfer is localized within few monolayers at the interface. With this assumption they linearized the self-consistent corrections to the on-site orbital energies. They found that the junction properties depend essentially on the characteristics of the first few metal layers and based on this argument they analyzed the variation of the Fermi level as a function of metal coverage. They identified strong- and weak-coupling regimes for the metal-semiconductor interaction and calculated values for $\phi_{B n}$ and $S$, which were close to the observed ones.

An alternative model for SB formation was proposed by Tersoff[9] based on the metallic screening 
of MIGS. Tersoff claimed that the model is universal and aims at determining a canonical barrier height as a function of only the semiconductor band structure. In turn this canonical barrier height determines the pinned position of the Fermi level in a SB. To this end, he argued that the density of states of MIGS is large enough to avoid any deviation from the local (layer-by-layer) charge neutrality, since such a deviation requires an immense electrostatic energy. Consequently, the Fermi level is forced to stay at or close to the charge neutrality level as in Eq. (7). Tersoff also proposed a simple method for obtaining the charge neutrality level (developing this method independently he called this energy level as the branch point $E_{B}$, for the sake of uniformity we refer to it as $\phi_{o}$ as above since they have the same meaning) and gave a qualitative argument for the Schottky-like behavior of ionic semiconductors.

For one-dimensional semiconductors it is known that the charge neutrality level corresponds to the branch point of energy in complex band structure. That is, exactly at this energy the weight of virtual states comes equally from the valence and conduction bands. The cell-averaged real-space Green's function

$$
G(\vec{R}, E)=\int d^{3} r \sum_{n \vec{k}} \frac{\psi_{n \vec{k}}^{*}(\vec{r}) \psi_{n \vec{k}}(\vec{r}+\vec{R})}{E-E_{n \vec{k}}}=\sum_{n \vec{k}} \frac{e^{i \vec{k} \cdot \vec{R}}}{E-E_{n \vec{k}}}
$$

changes signs at $E=\phi_{0}$ for sufficiently large $|\vec{R}|$ ( $\vec{R}$ being the lattice vector) in one-dimension. Here $n$ and $\vec{k}$ are the band index and Bloch wave vector, respectively, and $\psi_{n \vec{k}}$ and $E_{n \vec{k}}$ are the corresponding wave function and energy. Exploiting this fact, Tersoff disputed that in threedimensions the relevant charge neutrality level for a semiconductor is the one found by taking $\vec{R}$ normal to the interface. Stressing the independence of the SB height from orientation he argued that the most important direction is the one which yields the smallest decay constant leading to a strong metallic (Thomas-Fermi) screening. In addition, he commented that for ideal epitaxial interfaces the face dependence of the SB height should be observed experimentally. Using the procedure outlined above he calculated $\phi_{0}$ for a variety of elemental and compound semiconductors. Later Tersoff[33] investigated the ionic-to-covalent transition by calculating the decay lengths of MIGS. He found that for covalent or weakly-ionic semiconductors it is large enough to screen the interface dipoles, but for highly ionic semiconductors the MIGS decay quickly (or equivalently the density of MIGS is small) so that the dipole due to electron affinity difference can not be screened effectively.

Although Tersoff $[9,33]$ noted that there are systematic variations between the experimental results and canonical barrier heights, he commented that due to its simplicity and the fact that it is strictly dependent on the semiconductor, this method can be used for predictive purposes to 
within $\pm 0.1 \mathrm{eV}$ for typical systems. This point has attracted attention and usually the explanation by Tersoff has been recognized as the MIGS model. Nevertheless, some recent experimental results suggested that this simple explanation may fail even when the necessary conditions are satisfied[5].

Harrison[34] focused attention on the metal-semiconductor bonds at the interface and the dipole layer associated with these bonds by using a self-consistent tight-binding method to deal with semiconductor heterojunctions. He argued that the SB formation might be studied by using the same method. To this end, he suggested to change the metal by an image lattice of the semiconductor. The calculated natural band line-ups put the Fermi level of the metal high in the conduction band and the dipole corrections (calculated in this image approximation) did not lead to reasonable barrier heights. Thus he concluded that it was necessary to include the dipole corrections of MIGS. He found that the largest contribution is due to the heavy-hole bands, but the total dipole shift is still small to obtain the experimentally observed barrier heights.

Later Harrison and Tersoff[35] reevaluated the tight-binding theory of interface dipoles. Their self-consistent calculations showed that it is the natural band discontinuity which is screened in the semiconductor, and not the dipole formed due to the charge transfer. On this ground they recalculated the SB height and found that it was pinned at the average hybrid energy of the semiconductor. This is the charge neutrality level for this tight-binding model[33,35]. Their results were in agreement with the general trend of the experiments. Same authors studied[36] the transition-metal impurities in semiconductors. They argued that due to the large electron-electron repulsion (much larger than the hybridization energy) the transition-metal atom has minimum energy state when it is neutral. Thus, the host crystal, as well, has to attain its charge neutrality. This puts the impurity levels of the transition-metal atoms exactly on the charge neutrality level of the semiconductor, so they are correlated with the SB heights.

\section{F. The defect models}

Theories discussed so far assumed the presence of a thick metallic overlayer. With the advent of experimental techniques[5] (growth mechanisms and measurement techniques, especially photoelectron spectroscopy) it has been possible to observe the evolution of the SB height as a function of metal coverage. A number of such studies by Spicer and coworkers[10] yield results affirming that the Fermi level is stabilized even for submonolayer coverages, that is when it is not possible to talk about a metallic overlayer. Two main conclusions of these studies were the independence of the position of the Fermi level from the metal used and the pinning at very small 
density of metal induced states (usually submonolayer coverages).

Spicer et al.[10] proposed that a possible source for having such a pinning is the defect levels of the semiconductors. They argued that the chemical reactions taking place at the interface give rise to release of energy which is enough to form some native defects at the semiconductor side. These complicated chemical reactions also lead to disruption of the semiconductor surface, which in turn form deep defect levels in the semiconductor band gap. Recalling Bardeen's estimate[6] that only a small density of states (on the order of $10^{13} \mathrm{~cm}^{-2}$ ) is enough to pin the Fermi level and also considering the pinning at submonolayer coverages, they concluded that these defect levels determine the SB height. Namely,

$$
\phi_{B n}=E_{g}-E_{d}
$$

where $E_{d}$ is the energy of the defect level responsible for pinning. It was also argued that similar pinning effects for nonmetallic overlayers (e.g., oxygen) require an explanation independent of the metallic character of the overlayer. One of the important aspects of this model is that, the pinning is related to effects extrinsic to the interface, that is to the properties of the semiconductor. We will refer to this model and the related ones as the defect model hereafter.

The observation of two different pinning positions for $\mathrm{n}$ - and $\mathrm{p}$-type semiconductors for certain metals lead to conclusion that both donor and acceptor type defects have to exist. This pinning mechanism was called the Unified Defect Model[10,37] (UDM). For binary alloys of homopolar semiconductors the dangling bond states[38] were shown to be in quite good agreement with the measured barrier height. These dangling bonds can be formed around the voids, vacancies and disordered regions, a few monolayers inside the semiconductor so that they are not screened by the metal charge. On the other hand for III-V semiconductors and their pseudobinary alloys the anion and cation dangling bonds[39] could not explain the pinning. For these surfaces the anti-site defects[39] and vacancies[40] are likely to reproduce the observed barrier heights.

The defect model for SB formation has received both recognition and serious criticism in the last decade. The experiments carried out with submonolayer and a few monolayers of metal coverages justified the relevance of the UDM to the SB, and the necessity of including extrinsic effects in a complete theory. As pointed out by Sankey et al.[38] and later by Lindau and Kendelewicz[11] in an extended review, the MIGS model neither aims at nor explains the pinning observed at submonolayer coverages. Lindau and Kendelewicz[11] summarized the points to be investigated in the UDM. They argued that both chemical reactions and cluster formation are exothermic reactions, creating defects at the interface which can migrate into the semiconductor where metallic screening is ineffective. They pointed out the importance of the interfacial chemistry, that is, the 
differences between reactive and unreactive metals. Another important problem is the analysis of the effects of thermal aging on thick diodes. The difficulty arising in examining the defect levels is twofold. First, there are a large number of possible defect levels lying close to each other in the energy gap 80 it is not straightforward to assign a specific defect to a pinning position of the Fermi level. That is, the predictive value of the theory is not high. Secondly, for most cases the defects are not easy to probe experimentally, thus the justification of the arguments based on the defect model is not easily achievable. Similarly, the absence of pinning for defect-free interfaces[33] (equivalently the complete omission of MIGS) have been the major drawback of the UDM mechanism.

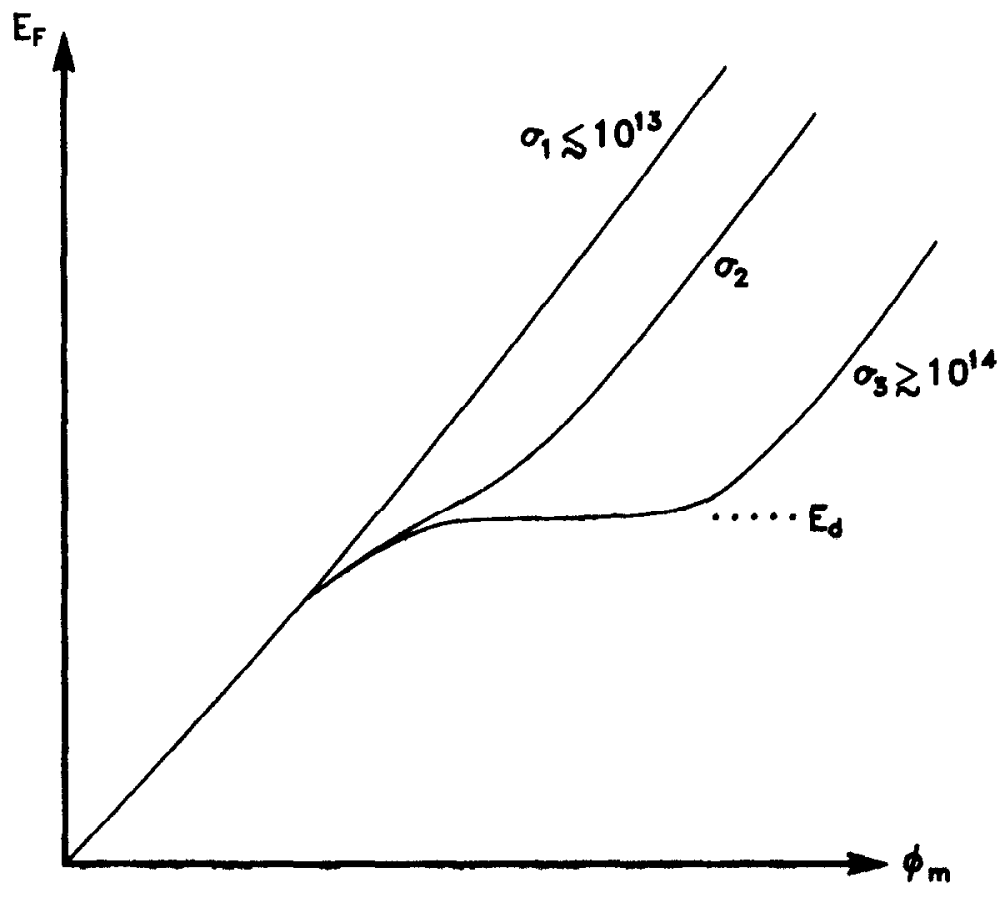

Fig. 4. Position of the Fermi level as a function of the metal work function. The effect of both the defects and metallic screening have been taken into account. $E_{d}$ denotes the defect level.

The first microscopic consideration of the defect model was done by Zur et al:[41]. They modeled a thick Schottky diode within the jellium approximation and the defects were assumed to be localized in the semiconductor a few angstroms from the interface. In contrast to Bardeen[6] they found that the density of defects that is necessary to stabilize the Fermi level is at least one tenth of a monolayer $\left(10^{14} \mathrm{~cm}^{-2}\right)$ due to screening by the metal. In addition to this, the pinning is 
not robust against varying metal work function. Therefore the barrier height resembles to that found by the Schottky model, with some shoulders and pinned portions as shown in Fig. 4. They calculated the coverage dependent position of the Fermi level for submonolayer metal coverage as well, by removing the jellium representing the metal and using only defects near the semiconductor surface to simulate the effect of submonolayer metal deposition. They found that the barrier height evolves symmetrically for $\mathrm{n}$ - and p-type substrate if there are deep donor and acceptor defect levels placed symmetrically in the semiconductor band gap. This result was in agreement with the experiments which have been carried out until then. A similar method recently was used by Mönch[25] to investigate the mixed effects of the MIGS and defect levels for Si based SB. He used the effective metal electronegativities as given by Miedema, instead of the metal work function, and analyzed several metal and silicide overlayers. He concluded for abrupt interfaces the barrier height is determined solely by the MIGS model. On the other hand for highly defected surfaces he identified two defect levels with densities approximately $10^{14} \mathrm{~cm}^{-2}$ located 0.62 and $0.37 \mathrm{eV}$ below the bottom of the conduction band of $\mathrm{Si}$. He carried out similar calculations for GaAs interfaces as well. In agreement with the UDM he concluded that for defective interfaces a high density defect level is located $0.65 \mathrm{eV}$ below the conduction band. These studies showed that for thick diodes the defect model alone is not an appropriate explanation, but it has to be supplemented by the metallic screening effects.

One of the important achievements in the experimental analysis is the comparative study of metal deposition at low $\left(\sim 80^{\circ} \mathrm{K}\right)$ and room temperatures. For some metals the reactivity can be greatly reduced at low temperatures which leads to the MIGS model for the SB height. The position of the Fermi level in the semiconductor band gap is shown in Fig. 5 for typical cases. However, for some metals the chemical reactions are possible even at low temperatures so that the barrier height is consistent with the defect model. Analyzing the outcomes of a large collection of experimental results, Spicer and coworkers[37] confirmed that depending on the reactivity of the metal, growth temperature and semiconductor used, either the defect or MIGS model or a combination of two has to be considered for a correct explanation of SB formation. In addition to that, it was argued that even in the presence of a thick metallic overlayer the defects affect the barrier height. Therefore, the evolution is not an abrupt transition between the defect and the MIGS models, but a detailed balance exists between the two mechanisms.

For most of the systems it was found that the submonolayer evolution of the Fermi level was considerably changed at low temperature. Most importantly the symmetry of the $\mathrm{n}$ - and p-type semiconductors was demolished at low temperature[5,37]. For n-type materials the Fermi level does 


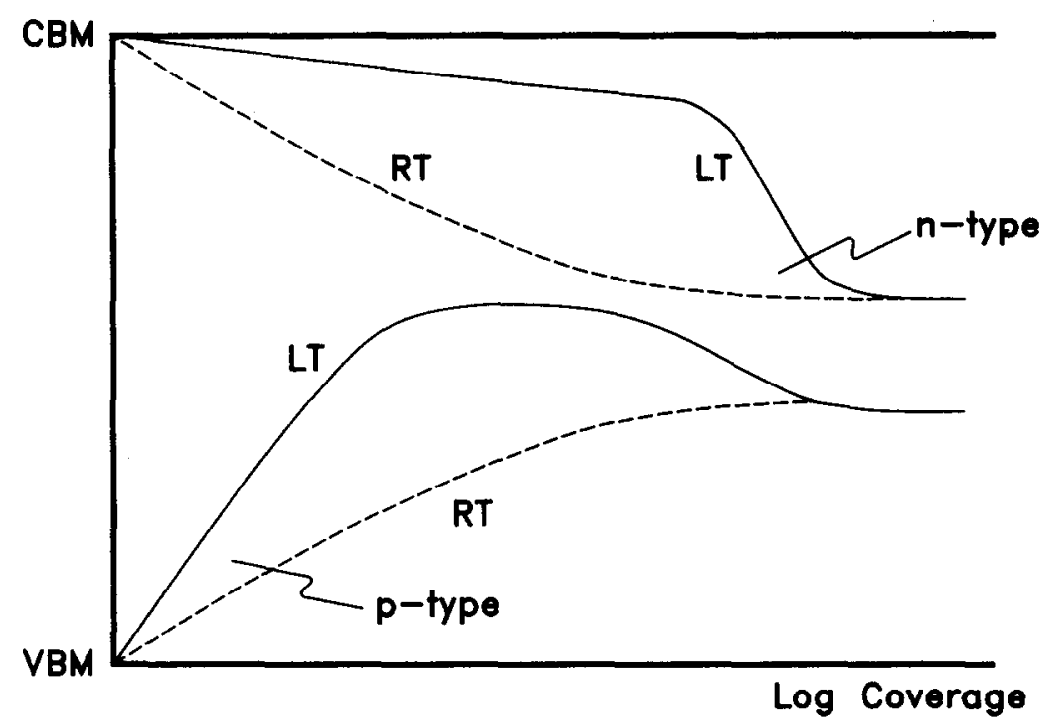

Fig. 5. Position of the Fermi level in the semiconductor band gap as a function of the metal coverage (logarithmic scale). Full (dash-dotted) curve corresponds to low temperature, LT, or unreactive (room temperature, RT, or reactive) behavior. The shown curves are typical ones and exceptions do exist. CBM (VBM) denotes the conduction band minimum (valence band maximum).

not move much until the overlayer becomes metallic and then dives to its final pinning position. On the other hand for p-type materials the Fermi level increases rapidly and around a few percent monolayer coverage it is pinned at a level which is usually a bit higher than the final pinning position. These results justified the importance of the defect levels for submonolayer coverages. However, the asymmetric behavior can not be reconciled by the UDM, since this requires an unphysical difference among the rates of donor and acceptor formation.

Thus, Spicer and coworkers[12] proposed a more elaborate explanation for the defect mechanism, which was called the Advanced Unified Defect Model (AUDM). They pointed out that for GaAs interfaces the pinning position of the Fermi level was in quite good agreement with the energy positions of the Asas antisite defect levels. Note that, the UDM for GaAs assumes the presence of these double donor defects only[39]. Nevertheless, the low coverage pinning for p-type semiconductor requires the existence of a minority acceptor defect as well. Based on this result they suggested that the minority acceptors are $\mathrm{Ga}_{\mathrm{As}}$ antisites, densities of which was found to be approximately half that of $\mathrm{As}_{\mathrm{Ga}}$. This was consistent with the As-rich character of the interface 
since the free $\mathrm{Ga}$ atoms diffuse out of the interface as justified experimentally.

Recently Doniach and coworkers[42] investigated the effect of microscopic metal clusters on Schottky barrier formation using a semiclassical approximation. They included the screening of the charge trapped in the defect levels by the free charge in the clusters and the Coulombic interaction between the two. Based on the numerical results they concluded that the model can explain the room temperature behavior of the Fermi level as a function of metal coverage. For low temperature case the electronic properties of the small metal clusters are differing appreciably form those of large ones which is used for room temperature calculations. They attributed the overshoot observed for p-type semiconductors at low temperature to a crossover from small clusters to the larger ones.

\section{G. Adsorbate-induced states}

The coverage dependent evolution of the Fermi level at low temperature was also analyzed by Mönch[25] based on the assumption that some adsorbate-related surface states of donor type are formed at the interface due to metallization. In contrast to original defect models this is an intrinsic mechanism and depends on the type of metal atom as well. Earlier, Ciraci and Batra[43-45] drew attention to the fact that different mechanisms may determine the position of the Fermi level at different metal coverages. They argued that while adsorbate-induced (or chemisorption) states become dominant at about monolayer coverage, at thick coverages MIGS and perhaps defect levels are responsible for the pinning. Mönch[25] found that the submonolayer overshoot values of the Fermi level position in p-type semiconductors and the first ionization energies of the adsorbate atoms correlate linearly. He found that the initial overshoot observed for p-type materials can be explained assuming that these adsorbate-induced states act as donors. In contrast to the AUDM, the disappearance of the overshoot is related to the transition of adsorbate-induced surface states to the MIGS. In fact such a behavior of $\mathrm{Cs}$ on $\mathrm{Si}$ was studied by Heine[7] in his original paper.

Kahn et al.[46] studied the evolution of the Fermi level at both low and room temperature by giving emphasis to the metallization of the overlayer. They gave estimates for the cluster size and morphology of the metal overlayers based on the photoemission results. Their results confirmed the adsorbate-induced surface states explanation of Mönch[25] for low coverages at low temperature. The abrupt changes in the Fermi level position at higher coverages are shown to appear exactly at the threshold of overlayer metallization. Thus they attributed the final barrier heights to pinning by the MIGS. The temperature dependence of the evolution of the Fermi level 
position was associated with the growth mechanism of the overlayer.

The consensus about the screening effect of the metallic charge was questioned by Ludeke[13,14]. Experiments performed on intentionally defected interfaces[13] gave evidence that the simple screening picture may be incomplete. It was shown that a metal overlayer which is sufficient to pin the Fermi level for defected surfaces may yield to completely different pinning positions after annealing the defects. Ludeke suggested that the main effect of the metal overlayer is the delocalization of the defect level. Without the continuum of the metal states, the defects have discrete localized states so that the occupancy of these states is either zero or one. When this system is brought into contact with a metal the tunneling between the defects and metal give rise to broadening of the defect levels and formation of a continuum of states which may have noninteger occupation. The amount of this charge $q_{\mathrm{eff}}$ is determined by the position of the Fermi level which in turn is related to the strength of the total dipole at the interface. Therefore the occupancy of the delocalized defect levels has to be determined self-consistently. Using the UDM for the defect level Ludeke found that for low work function metals $q_{\text {eff }}$ may be large leading to a complete pinning of the Fermi level. However, for metals having electronegativity close to that of the semiconductor $q_{\text {eff }}$ is around 0.1 electronic charge and the SB height changes almost linearly with $\phi_{m}$. However, since the MIGS were omitted completely the model applies only to a case where a very large density of defects is present. Using the same approach the index-of-interface was also calculated and was shown to be consistent with the experiments[13] for a wide range of semiconductors.

Experiments by Aldao et al.[15] gave support to the explanation by Ludeke[13,14]. They studied the dependence of the Fermi level movement to the doping concentration of the semiconductor at low temperature. For high dopant densities $\left(\sim 10^{18} \mathrm{~cm}^{-3}\right)$ the evolution of the SB height was shown to be quite different than that for lower dopings for a variety of metals. The naive expectation for a stronger screening of defects and thus for a strong pinning of the Fermi level was in complete disagreement with these results. This anomalous behavior was attributed to delocalization of the adsorbate-induced states due to their interaction with the semiconductor. Although for this case the broadening was related to the metallic character of the semiconductor, the essence of this effect is the same as that proposed by Ludeke[14]. These studies pointed out that the role of the continuum of metal states is not clarified yet in spite of the common agreement on the screening effects.

Recently Masri[47] proposed an alternative approach to the adsorbate-induced states in terms of two-state systems. In this explanation metal-like states and metal-induced semiconductorlike 
states are attached to each other in the form of pairs. For low coverages these states are reminiscent of the adsorbate-induced states. With increasing coverage they evolve into a continuum which, in turn, gives support to MIGS mechanism. Masri used a one-dimensional tight-binding model to exemplify the two-state nature of the M-S system. However, this model has not been analyzed in detail yet to judge on its applicability to SB problem.

\section{Simple Metal-Semiconductor Interface}

In the previous section we discussed several features which significantly affect the properties of metal-semiconductor interfaces. In particular, the type of metal (simple, transition, or alkali metal) and the type of semiconductor (elemental or compound semiconductor) were found to be essential in the mechanism of pinning the Fermi level.

The first self-consistent electronic structure calculations on the metal-semiconductor interface were done by Louie and Cohen[29] by using repeating slab model and representing the metal overlayer by a jellium. They thus neglected the lattice incommensurability between the $\mathrm{Al}$ and $\mathrm{Si}(111)$ surfaces and used periodic boundary conditions to represent the wave function in terms of plane waves. Despite the artifacts of the jellium metal, their results provided significant insight about the states near the Fermi level. In fact, their self-consistent field (SCF) pseudopotential calculations revealed states which are bulk-like in the jellium and dangling-bond like at the interface, but decay into Si. Later, Batra[48] pointed out the Al-Ge(001) interface, which is nearly lattice matched, allows the study of the M-S interactions on the atomic scale.

In this section, we concentrate on the simple metal-semiconductor junction between a simple metal (Al) and an elemental semiconductor (Ge) allowing a nearly lattice matched interface. Pseudomorphic growth[49] of $\mathrm{Al}(001)$ film on a $\mathrm{Ge}(001)$ substrate has been achieved by molecular beam epitaxy. To achieve lattice matching the $\mathrm{Al}(001)$ slab must be rotated $45^{\circ}$ with respect to the ideal $\mathrm{Ge}(001)$ lattice. For this case the lattice mismatch is only $1.2 \%$, which can easily be accommodated by the lattice strain. Since the interface is perfect, several factors (such as interface defects, interface reconstruction, etc.) are eliminated, and the nature of the M-S interaction is examined on the atomic scale. The ab-initio calculations, which provide valuable information on the character of the electronic states near the Fermi level and the energetics of the interface, can be performed with reasonable success. In this section, we examine the character of the states near the Fermi level of the Al-Ge interface. 


\section{A. Submonolayer coverage and overlayer metallization}

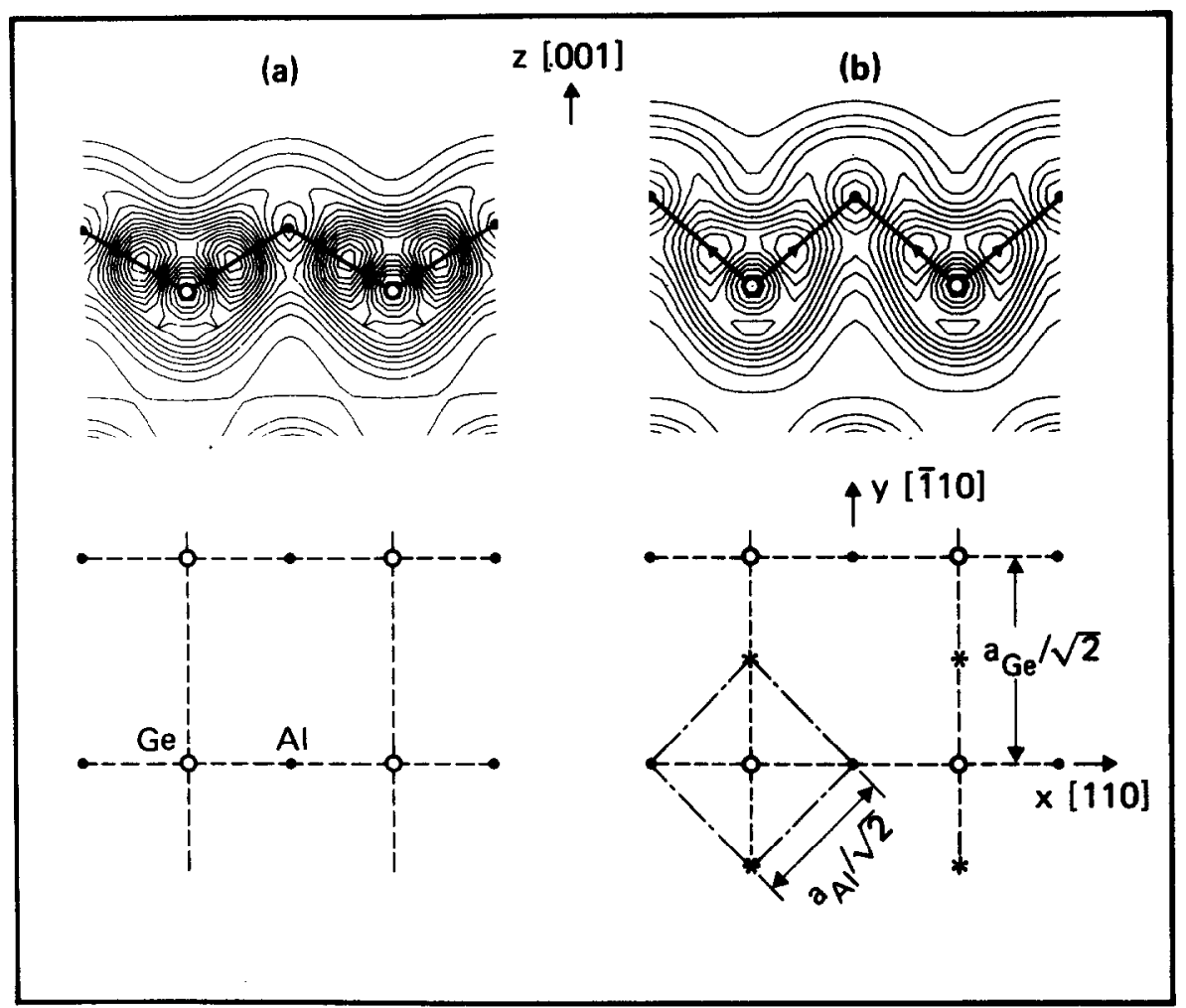

Fig. 6. Charge density contours in the (110) plane of the $\mathrm{Al}(001)-\mathrm{Ge}(001)$ interface corresponding to aluminum in bridge-bonded sites for (a) $\theta=1$ and (b) $\theta=2$. The contour spacings are 0.005 electrons/a.u. ${ }^{3}$ Atomic arrangements are also shown for the two coverages; the stars and filled circles represent $\mathrm{Al}$ atoms, and open circles Ge atoms. (Reproduced from Ref. [45]).

The SCF-total energy calculations[44,45,48] were carried out for five layers of the ideal Ge(001) slab (representing the semiconductor substrate) and absorbed $\mathrm{Al}$ atoms starting with the coverage $\theta=1$ [i. e., one $\mathrm{Al}$ atom absorbed on each $\mathrm{Ge}(001)$ surface unit cell] up to multilayer $\mathrm{Al}$ coverage. Figure 6 illustrates the interface atomic configuration for the metal coverage $\theta=1$ and 2 , and the charge density of the Al-Ge bonds corresponding to these coverages. The adsorption site for 


\section{I.P. Batra et al.}

1 is determined by the structure optimization, which favors the bridge site providing a natural ridization of $\mathrm{Al}-s p^{2}$ orbitals with the surface dangling bonds. The binding energy per adsorbed n

$$
E_{b}=E_{T}[\mathrm{~S}]+E_{T}[\mathrm{M}]-E_{T}[\mathrm{M}+\mathrm{S}]
$$

sbtained from the total energies of the metal-semiconductor $(M+S)$ system, the bare iconductor (S) and the metal atom (M), the positive values of which being energetically rable. The adsorbed atom at the bridge site and $h=2.3$ a.u. above the semiconductor ace formed strongest bond for $\theta=1$ with a binding energy of $3 \mathrm{eV}$. At $\theta=2$, the second atoms located at the other bridge site and in the same plane as the first $\mathrm{Al}$ atom form the 301) layer above the $\mathrm{Ge}(001)$ surface. In this case the binding energy per adsorbed $\mathrm{Al}$ atom seases compared to $\theta=1$, but the equilibrium occurs at relatively larger Ge-Al interplanar ance corresponding to $h=3.4$ a.u. This means that for $\theta=2$ the $\mathrm{Al}(001)$ layer rises to $h=3.4$ , and as a result of this the strongly directional Ge-Al bonds are elongated and become less lized. The delocalization of the M-S bonds can also be revealed from the analysis of the charge ribution. The maximum of the calculated SCF-charges, $\rho_{\max }$ for $\theta=1$ reaches a value of ' 9 a.u. in the middle of the Al-Ge bond; the corresponding value for $\theta=2$ is only 0.056 a.u. sesting a weaker bond. The weakening of the M-S bond at relatively larger metal coverage irs since $M-M$ coordination and the $M-M$ interaction increases with increasing coverage. Once etallic bonding is set in above the semiconductor surface some charge from the M-S bond (or a the interface) is transferred to the metal layer. We note that the overlayer metallization Irs for certain M-S systems since the total energy of the system is lowered to be a (local) imum on the Born-Oppenheimer surface. This usually takes place if the M-M interaction is nger than the M-S interaction.

he metallization of the Al overlayer subsequent to the weakening of the Al-Ge bonds is clearly wn in Fig. 7 by the charge density contour plots calculated for various degree of metal coverage. $\theta=1$, there is a valley parallel to the [110] direction showing lack of interaction between ninum atoms along [110] direction. However, the $\mathrm{Al}$ atoms located along the [110] direction $x$ highly directed interaction mediated through the substrate. It is seen that at low coverage formation of directional bonds is responsible for such anisotropic behavior leading to oneensional (1D) metallic chain. The complete metal overlayer $(\theta=2)$ shows a more isotropic rge density as is evident from Fig. 7(c). The aluminum layer shows a tendency towards allization, and chemisorption bonds with semiconductor are weakened.

he metallic behavior and dimensionality of the $M$ overlayer can be examined using the local 
$\uparrow[\overline{1} 10]$

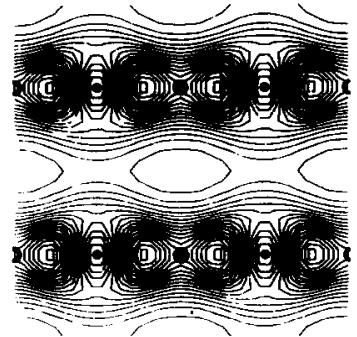

(a)

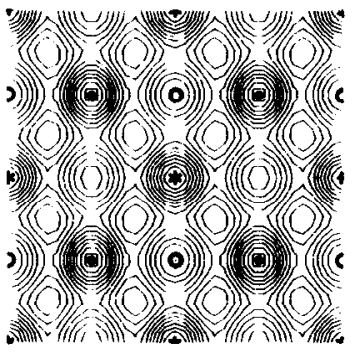

(c)

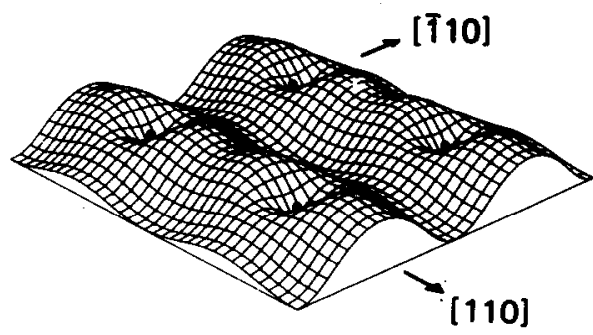

(b)

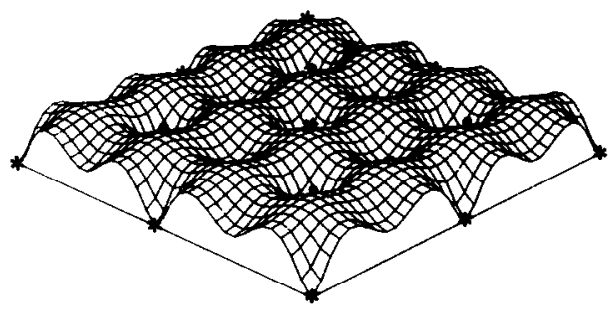

(d)

Fig. 7. Charge density contours (spacings 0.0025 electrons/a.u. ${ }^{3}$ ) and the surface maps in the (001) plane passing through aluminum atoms of the Al-Ge interface corresponding to aluminum atoms located in bridge-bonded sites for (a-b) $\theta=1$ and (c-d) $\theta=2$. Notice the transition from anisotropic chemisorption state to metallization of the overlayer. (Reproduced from Ref. [45]).

density of states (LDOS) calculated at the overlayer for two coverages under consideration. For $\theta=1$, the Ge-Al-Ge bridge bonds are produced by the bonding combination of Ge dangling bonds and Al-sp $p^{2}$ orbitals. The chemisorption states appear near the Fermi level and also at about $3 \mathrm{eV}$ below it. States having the Al-3py character would couple the metal atoms along the [110] direction. However, since these states are unoccupied, the Al overlayer displays a nearly $1 \mathrm{D}$ character. Whereas, for $\theta=2$, the LDOS may be viewed as a modulation on top of the ladder-type density of states expected for a 2D metal. The steps in the LDOS are reminiscent of the subband jumps of the unsupported quasi-2D free electron system. This implies that by increasing the $\mathrm{Al}$ coverage from $\theta=1$ to $\theta=2$, the overlayer changed into a quasi-2D metal. 


\section{B. Multilayer coverage and electronic structure}

As a continuation of the above analysis, we discuss the energetics of the interface and electronic states responsible for the pinning of the Fermi level at multilayer coverage by using the results of the SCF-pseudopotential calculations[50] carried out for $\mathrm{Al}_{10} \mathrm{Ge}_{5}$ superlattice corresponding to 5 layers of Al. To examine the effect of the overlayer thickness these calculations were also extended to thicker Ge-slab and thicker Al-overlayer corresponding to 7 layers of Al. The atomic arrangement for $\mathrm{Al}_{10} \mathrm{Ge}_{5}$ is illustrated in Fig. 8.

A comparison of the total energy of the superlattice with those of the bulk Al and Ge calculated alone gives additional insight into the M-S interaction at multilayer metal coverage. To this end, the total energy of the $\mathrm{Al}(\mathrm{Ge})$ slab are calculated by removing the $\mathrm{Ge}(\mathrm{Al})$ slab, but keeping the supercell and kinetic energy cutoff the same. The adhesion energy of the metal overlayer per interface defined as

$$
E_{a}=\left\{E_{T}[\mathrm{Al}]+E_{T}[\mathrm{Ge}]-E_{T}[\mathrm{Al}+\mathrm{Ge}]\right\} / 2,
$$

and found to be $2.24 \mathrm{eV}$. The total energy is lowered owing to Al-Ge bond formation at the interface. However, the calculated value of $E_{a}$ is smaller than the binding energy of the $\mathrm{Al}$ atom adsorbed at the bridge site at $\theta=1$. This reflects the weakening of the Al-Ge bond in the presence of the relatively stronger metallic bonding within the metal slab.

The calculated perpendicular components of the forces acting on the atoms on the metal and semiconductor atoms forming atomic (001) planes are not uniform (see Fig. 8), and hence indicates that neither the interlayer spacing nor the perpendicular displacements of metal atoms in a given layer are uniform[50]. This gives rise to the buckling of the atoms near the interface of Al-Ge even if it is nearly commensurate. The degree of buckling, however, decreases farther away from the interface.

The formation of the M-S bonds is examined by considering the difference charge density

$$
\Delta \rho(\vec{r})=\rho_{\mathrm{AlGe}}(\vec{r})-\rho_{\mathrm{Ge}}(\vec{r})-\rho_{\mathrm{Al}}(\vec{r})
$$

obtained by subtracting the charge densities of free $\mathrm{Al}$ and $\mathrm{Ge}$ slabs from the charge density of the $\mathrm{Al}_{10} \mathrm{Ge}_{5}$ superlattice. In Fig. 9 we show the contour plots of the charge densities $\rho_{\mathrm{Al}}(\vec{r}), \rho_{\mathrm{Ge}}(\vec{r})$, $\rho_{\text {AIGe }}(\vec{r})$ and $\Delta \rho(\vec{r})$ calculated in $(100)$ and $(010)$ planes. The $\Delta \rho$ plots clearly show the formation of M-S bonds. The charge depleted near the center of the dangling bonds and from the adjacent $\mathrm{Al}$ atom is accumulated near the center of the Al-Ge bond. In the (100) plane an M-S bond forms although the $\mathrm{Al}$ atoms in position $3^{\prime}$ do not continue the $\mathrm{Ge}-\mathrm{Ge}$ bond sequence between the 
(a)

(b)
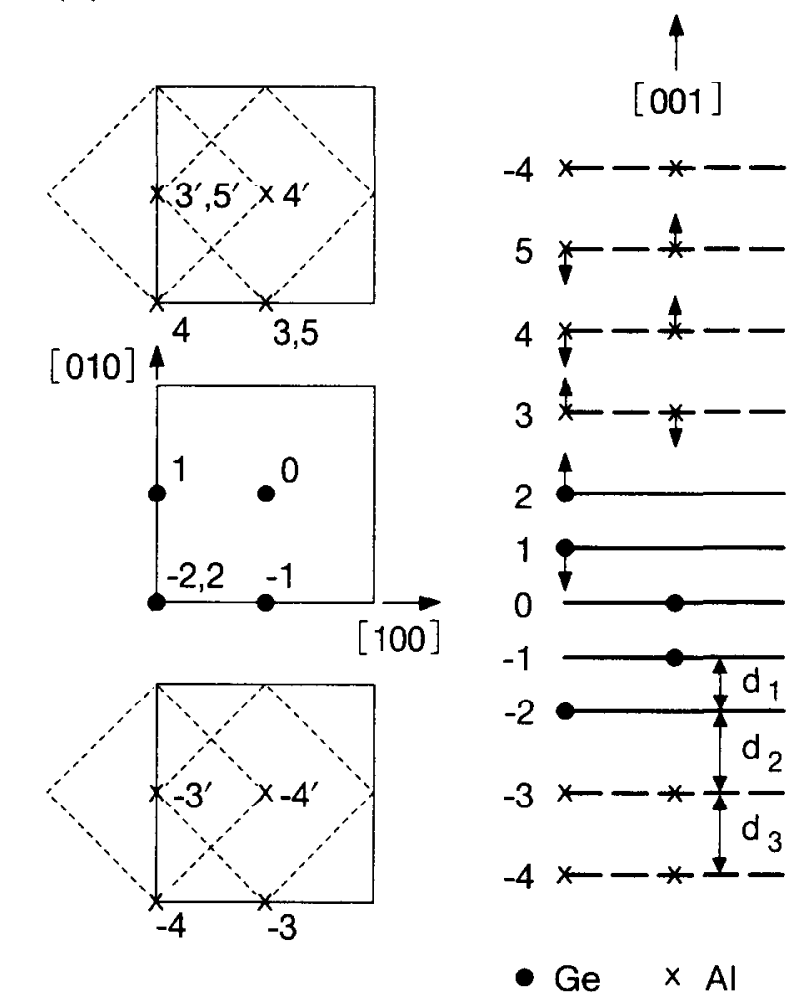

Fig. 8. Atomic arrangement in the $\mathrm{Al}_{10} \mathrm{Ge}_{5}$ supercell. (a) Top views from the [001] direction; inequivalent positions of metal atoms in the same layer are distinguished by primes. (b) Side view in the [010] direction; atomic planes are labeled by numerals. Arrows perpendicular to these planes indicate the directions and relative magnitudes of the forces calculated for the shown atomic arrangement. (Reproduced from Ref. [50]).

adjacent layers (see Fig. 8). As expected the M-S bond is stronger in the (010) plane, in which $\mathrm{Al}$ in position 3 has the tetrahedral coordination. Comparing the bond in the (001) plane with that which forms at $\theta=1$ (i. e., for a single adsorbed $\mathrm{Al}$ atom per $\mathrm{Ge}(001)$ unit cell at $h=2.3$ a.u.) it is, therefore, concluded that in the presence of a thick metal overlayer the M-S bond in the interface become weaker and less localized, but the delocalization is not complete and thus the directional covalent character of the bonds is maintained.

The evolution of the electronic structure upon junction formation and the character of the M-S 


\section{I.P. Batra et al.}

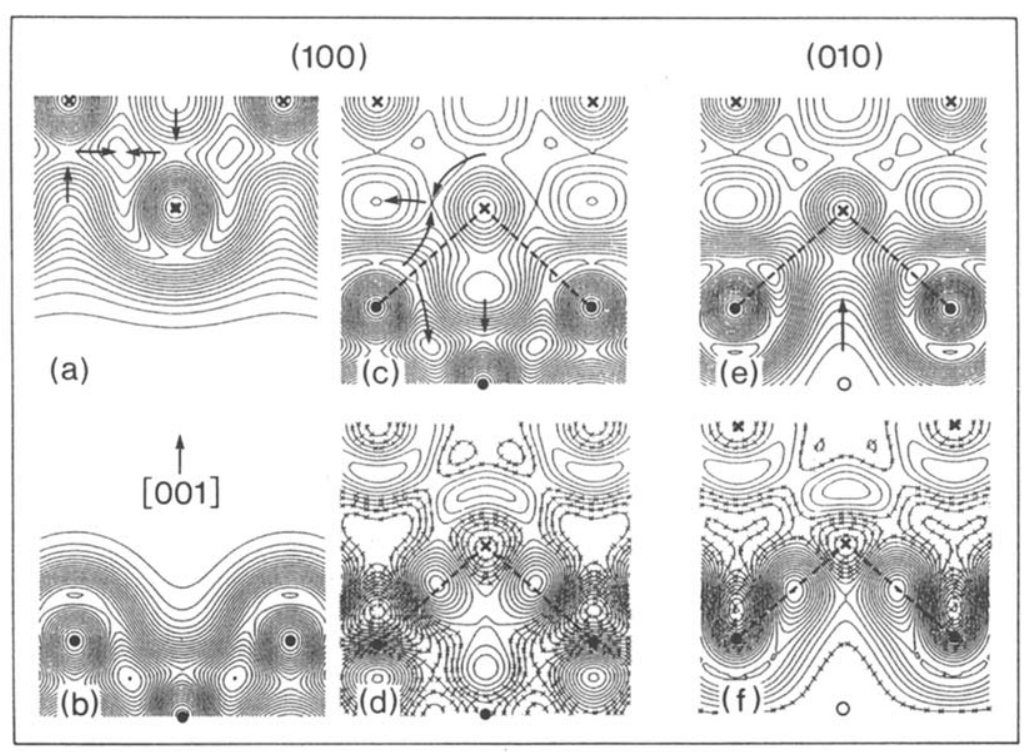

9. Contour plots of total and difference (AlGe-Al-Ge) valence charge densities. (a) Al (b) Ge slab. (c) $\mathrm{Al}_{10} \mathrm{Ge}_{5}$ superlattice with central $\mathrm{Al}$ at position $3^{\prime}$ as described in Fig. 8. iorresponding difference charge density. (e) $\mathrm{Al}_{10} \mathrm{Ge}_{5}$ superlattice with central $\mathrm{Al}$ at position 1 Corresponding difference charge density. Contour spacings are $15,33,33,6.8,27,8.2$ ${ }^{-4}$. electrons/a.u. ${ }^{3}$, respectively; arrows indicate directions of increasing charge density. ours with crosses refer to negative values. (Reproduced from Ref. [50]).

$s$ and other states with energies within or near the gap are analyzed by examining the total nterlayer densities of states, $D(E)$, and $L(E)$, respectively[50]. The interlayer density of states een the layers $\ell_{i}$ and $\ell_{i+1}$ is defined as

$$
\left.L_{\ell_{i+1}, \ell_{i}}(E)=\int_{\ell_{i}}^{\ell_{i+1}}\left\{\sum_{n \vec{k}} \psi_{n \vec{k}}^{*} \vec{r}\right) \psi_{n \vec{k}}(\vec{r}) \delta\left(E-E_{n \vec{k}}\right)\right\} d \vec{r},
$$

that the integral $\int^{E_{F}} L_{\ell_{i+1}, \ell_{i}}(E) d E$ yields the amount of charge between the layers $\ell_{i}$ and

;ure 10 shows total and interlayer densities of states which were calculated for the $\mathrm{Al}_{10} \mathrm{Ge}_{5}$ :lattice. The interlayer density of states is Ge-like between layers 0 and 1 (see Fig. 8 for labeling e layers), but exhibits important metal and semiconductor-like features in the interface (i. e., een layers 2 and 3 ). On the metal side (between layers 3 and 4) it becomes metal-like. It d be noted that $L_{0,1}$ has a finite density of states near $E_{F}$ (or in the gap) due to the Gaussian lening. 


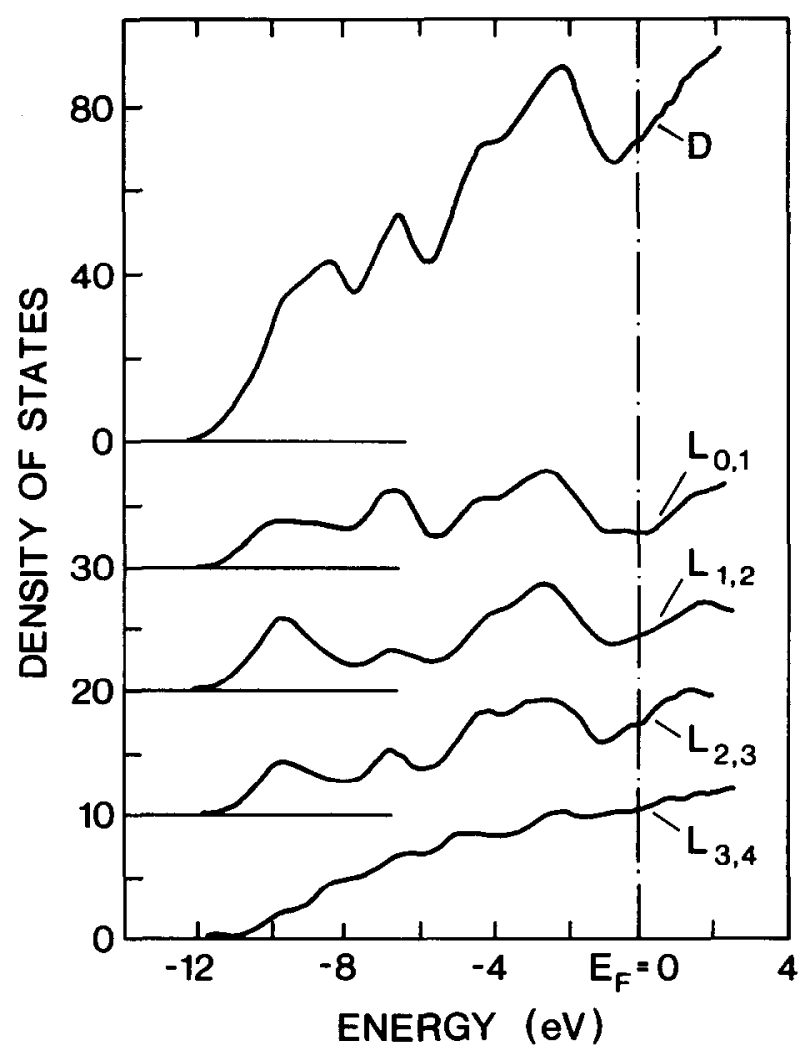

Fig. 10. Total $(D)$, and interlayer $(L)$ densities of states calculated for the $\mathrm{Al}_{10} \mathrm{Ge}_{5}$ superlattice. Subscripts indicate the layers specified in Fig. 8 between which the interlayer density of states has been calculated; thus $L_{2,3}$ denotes the Al-Ge interface. (Reproduced from Ref. [50]).

The total density of states of an $\mathrm{Al}_{10} \mathrm{Ge}_{5}$ superlattice is compared with those of the metal and semiconductor slabs alone in Fig. 11. The contribution of the surface states (shown by dotted lines) to the density of states of the Ge slab is obtained by integrating from the surface Ge-layer to the vacuum. The surface contribution at $E_{F}$ originating from the intrinsic dangling bond surface states exhibits a strong peak. The difference state density, i. e., $\Delta D=D^{\mathrm{AlGe}}-D^{\mathrm{Al}}-D^{\mathrm{Ge}}$ indicates that, upon junction formation, new states appear near -4 and $-11 \mathrm{eV}$ below $E_{F}$, whereas Gerelated features disappear near $0,-6$ and $-9 \mathrm{eV}$. The nature and origin of those changes are clarified by considering the difference interlayer densities of states, i. e., $\Delta L=L^{\mathrm{AlGe}}-L^{\mathrm{Al}}-L^{\mathrm{Ge}}$ which were calculated at the interface and in the adjacent regions above and below it. Dramatic 
changes occur at energies corresponding to M-S bonds $(-4$ and $-10 \mathrm{eV})$ and those of dangling bonds and backbonds (from $-2 \mathrm{eV}$ to $E_{F}$ ) of the Ge slab. Intrinsic surface states of the free Ge slab are modified and shifted out of the band gap to form M-S bonds. No significant changes occur between the surface and subsurface layers of the $\mathrm{Al}$ sublattice. The amount of charge calculated between the layers 2 and 3 (at the interface) is found to be approximately equal to the sum of those corresponding to the free $\mathrm{Ge}$ and free $\mathrm{Al}$ slabs calculated in the same region.

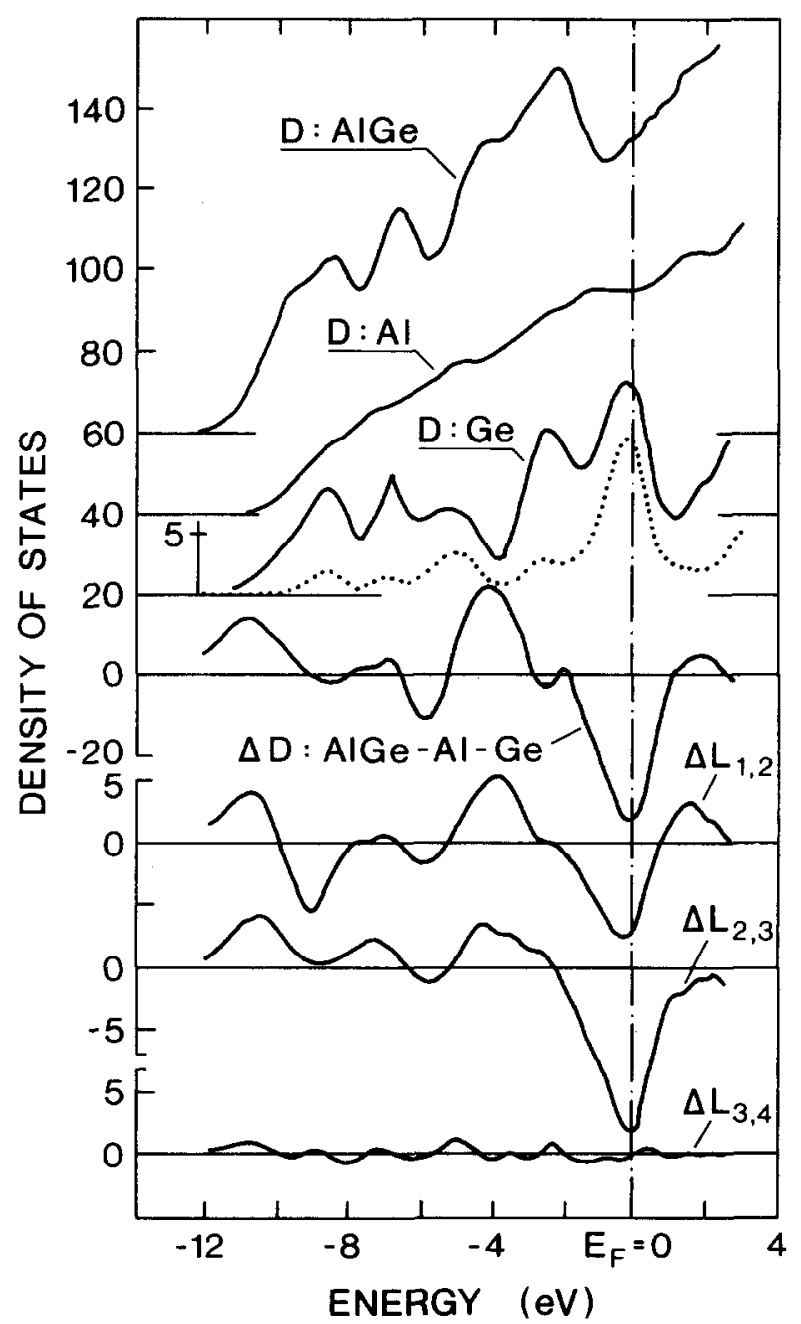

Fig. 11. Total $(D)$ densities of states calculated for the $\mathrm{Al}_{10} \mathrm{Ge}_{5}$ superlattice, for the free $\mathrm{Al}$ and Ge slabs, and surface contribution to the latter (dotted line with different scale), difference total density of states $\Delta D$ (AlGe-Al-Ge), and corresponding difference interlayer densities of states $\Delta L_{i, j}$ calculated between the layers $i$ and $j$. (Reproduced from Ref. [50]). 
Additional insight into the rearrangement of the electronic states upon formation of the M-S junction is obtained by subtracting the state density of the $\mathrm{Al}$ sublattice from that of the $\mathrm{Al}_{10} \mathrm{Ge}_{5}$ superlattice. Since the dangling bonds of the Ge slab are eliminated, the remaining state density is similar to that of the bulk Ge, except for some structure below $E_{F}$, due to the M-S bond. In the interface the corresponding interlayer density, (i. e., $\Delta L_{2,3}=L_{2,3}^{\mathrm{AlGe}}-L_{2,3}^{\mathrm{Al}}$ ) is found to be negligible near $E_{F}$. This implies that the states of the Al-Ge junction near $E_{F}$ are essentially derived from the $\mathrm{Al}$ metal. However, $\Delta L_{1,2}$ has a finite density near $E_{F}$. This is partly because some states leak down to the second layer of $\mathrm{Ge}$, and is partly an artifact of the broadening by a Gaussian.

The nature of states near $E_{F}$ can be revealed by examining the charge distribution of the individual states of the $\mathrm{Al}_{10} \mathrm{Ge}_{5}$ superlattice. Here, one has to keep in mind that for a lattice matched junction the wave vector parallel to the interface is still a good quantum number. Propagating solutions at either site can be matched into one wave function if their energies and wave vectors coincide. Such a situation can easily take place in the junction with a simple metal such as $\mathrm{Al}$, except for the states decaying into one side where they fall into a gap. For example, the lowest valence band state of $\mathrm{Ge}$ is formed from the bonding combinations of $4 \mathrm{~s}$ orbitals and occurs about $12 \mathrm{eV}$ below the common Fermi level. Since the width of the conduction band of $\mathrm{Al}$ is only $\sim 11 \mathrm{eV}$, the states of Ge having energies $-12 \leq E \leq-11$, decay into the metal. On the other hand, propagating metal states decay into the semiconductor if their energy falls in the gap of the semiconductor. While the states at the bottom of the Ge valence band are Ge-like in the corresponding sublattice, their weight is close to zero in the metal. In contrast, the state at the Fermi level is metal-like, but decays into the semiconductor, and hence has almost zero weight beyond the second layer in the Ge sublattice. These findings show that the qualitative description by Heine[7] is in good agreement with the real situation.

The metal states at the Fermi level which fall in the thermal gap of semiconductor are of particular interest. As mentioned in section 2, previously these states were designated metalinduced gap states (MIGS) and were found to be responsible for the pinning of the Fermi level. These states are expected to behave like intrinsic gap states (or dangling bond states) of Ge near the interface. However, this does not mean that Ge-dangling bond states (which were eliminated upon adsorption of the metal monolayer) reappear at thick metal coverage but are concealed in the band continua of Al. The bond formation illustrated in Fig. 9 and conclusions drawn from the difference density of states discussed in Figs. 10-11 also prompt us to reject the idea of reformation of dangling bond states in the presence of thick metal coverage. The metal states see the potential at the surface of semiconductor, which is different not only from the reconstructed but also from 
the ideal surface. The dangling bond states would turn to the resonance states and broaden in energy near $E_{F}$ if the metal side were a jellium with its edge terminated between $\mathrm{Al}$ and Gc atomic planes at the interface[29]. This is, however, an oversimplification omitting the M-S interactions on the atomic scale. The charge density plots of the $\mathrm{Al}_{14} \mathrm{Ge}_{9}$ superlattice presented in Fig. 12 display two types of states which are relevant for the M-S junction, namely MIGS and the semiconductor states decaying into the metal. Therefore the resonance state approach proposed by Lefebvre et al.[18] can not be taken as a proper explanation for the system at hand.

\section{Fermi level pinning at different coverages}

Above results are related to the lattice matched and perfect M-S interface. Here, one can draw the following conclusions relevant for the SB formation at the interface between a simple metal and an elemental semiconductor:

i) Although $\mathrm{Al}$ is a simple metal which usually displays a nearly free electron-like behavior, its interaction with $\mathrm{Ge}$ at the interface is on the atomic scale. Directional bonds are formed between $\mathrm{Al}$ and Ge atoms at the interface as a result of the significant charge rearrangement relative to the free surfaces. The interface bonding energy is significant, but is smaller than the binding energy of the single adsorbed $\mathrm{Al}$ atom, and smaller than the cohesive energy per atom of $\mathrm{Al}$ metal as well. This is explained by the transfer of charge from the Al-Ge interface bonds to the Al overlayer upon the onset of overlayer metallization. Consequently, the weakened bonds are elongated and the metal overlayer is raised above the Ge surface. This already occurs at the coverage of a monolayer corresponding to a 2D metal. Nevertheless, the M-S bonds are not completely delocalized as in the calculations that represent the metal overlayer by a jellium[29], but maintain their directional character.

ii) From analysis of the difference density of states, it is clear that the dangling bond surface states are eliminated upon the junction formation, and form new M-S states in the valence band. No evidence was found that the surface dangling bonds are maintained as resonance (or BreitWigner) although the weakening of M-S bonds at the interface do suggest such a possibility. Earlier calculations[29] yielded dangling bond-like gap states at the interface. Recent calculations[50,51] suggest that this was the artifact of the model, which represented the metal overlayer by a jellium with a sharp edge terminating halfway at the interface.

iii) For the Al-Ge interface, as well as some other M-S junctions the position of the Fermi level is determined by different types of states at different coverage values. At low coverage $\theta<2$ metal 


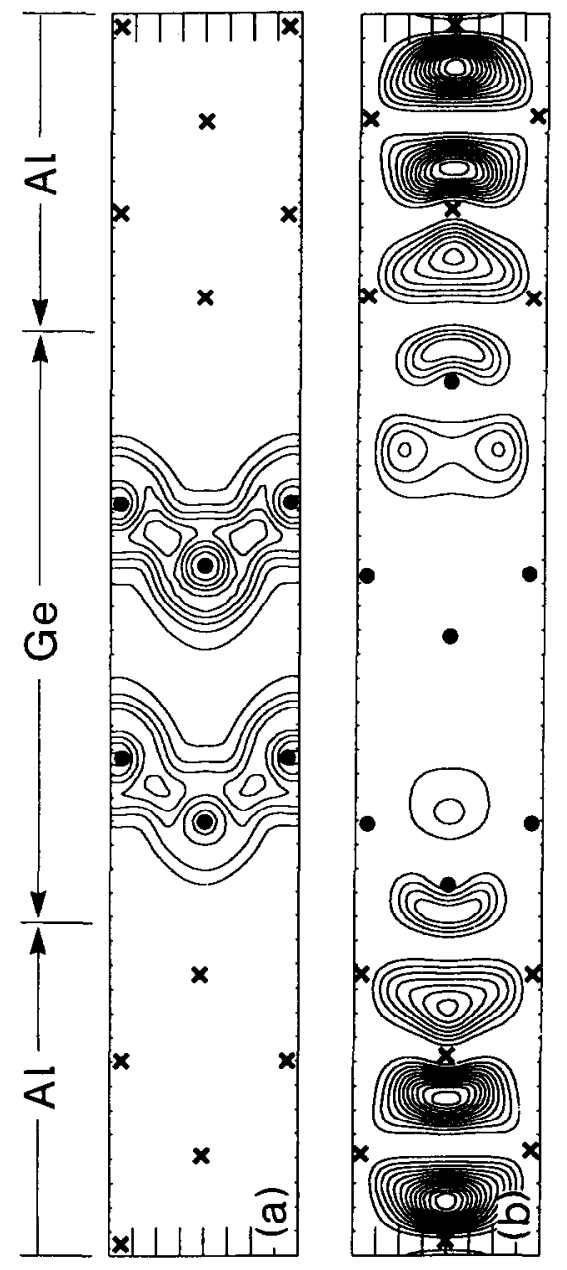

Fig. 12. Contour plots of the charge density of two typical states for $\mathrm{Al}_{14} \mathrm{Ge}_{9}$ superlattice shown in the corresponding supercell. (a) $E_{\Gamma}=-12.5 \mathrm{eV}$. (b) $E_{K}=-0.07 \mathrm{eV}$. Contour spacings are $10^{-4}$ electrons/a.u. ${ }^{3}$, and energies of the states are given relative to the Fermi level. $\mathrm{Al}$ and Ge atoms are indicated by crosses and dots, respectively. (Reproduced from Ref. [50]).

chemisorption states or adsorbate induced states determine the position of the Fermi level. Upon formation of broken or continuos adsorbate chains or layers the adsorbate-induced states may form a density states of finite width, which may overlap with the band gap of the substrate. Starting from monolayer the $2 \mathrm{D}$ metallic states develop. Near $E_{F}$, these states have high density in the metal film, but decay into the semiconductor. Their character is in compliance with the description in section 2 and therefore are identified as MIGS. The density of MIGS found in the present study is high enough to pin the Fermi level without invoking extrinsic states, such as defect or impurity 
states. Of course, this conclusion does not rule out the fact that the defect or impurity states can coexist with MIGS.

At this point, let us briefly comment on the recent work by Das et al.[52]. Using the linearmuffin-thin method within the local-density approximation, they carried out an extensive study of the epitactic $\mathrm{NiSi}_{2}-\mathrm{Si}(111)$ interface on the atomic scale. They found that the Fermi level is pinned by the interface states of the quasi-dangling bond character. These states occur because of the reduced $\mathrm{Si}$ coordination of $\mathrm{Ni}$ at the interface. $\mathrm{Bulk} \mathrm{NiSi}_{2}$ is a metal but has a partial gap for energies near $E_{F}$. In the $\mathrm{NiSi}_{2}-\mathrm{Si}(111)$ junction this gap partially overlaps the bandgap of $\mathrm{Si}$, and the band of the interface states runs through the central part of the common gap. In compliance with the arguments given in previous sections, the states, which fall into the common gap, decay on both sides and are localized at the interface. In addition, there are also metallic states at $E_{F}$ which fall into the bandgap of $\mathrm{Si}$ and have the character of MIGS.

iv) In his elaboration of Tersoff's theory[9], Lamıoo[18] identifies the charge neutrality level with the dangling bond energy $E_{d}$ at the free surface of the semiconductor. We note that $E_{d}$ is highly sensitive to surface structure and, in the case of some semiconductor surfaces, it can even dip into the band continua. Furthermore, the dangling bond surface states required in the model proposed by Lannoo may not exist at all for some M-S systems. For example, consider a $\mathrm{Ge}(001)$ or $\mathrm{Si}(001)$ surface which is saturated by a monolayer of $\mathrm{H}$ prior to metal deposition. Although the energy gaps of these surfaces are then free of the intrinsic surface states, the Fermi level can still be pinned by MIGS.

The charge neutrality level is relevant because a dipole owing to the transfer of charge between metal and semiconductor (or between two semiconductors in a heterostructure) gives rise to a dramatic increase of energy, which can not be balanced by the energy of bond formation. Therefore the electronic response to a certain interface structure in an M-S (or S-S) junction maintains the least amount of charge transfer to minimize the total cncrgy. If the assumed junction geometry leads to a significant charge transfer and thus an increase of the total energy, the interface atomic structure can even be forced to reconstruct in order to lower the total energy. A typical example is the $\mathrm{Ge}-\mathrm{GaAs}(001)$ interface. These two semiconductors are nearly lattice matched so that GaAs may be thought to grow as a natural continuation of the Ge lattice. However, such an ideal junction nevertheless requires charge transfer at the interface, resulting in a metallic system, in which the Fermi level overlaps with the tilted valence band continua of one the sublattices. The dramatic increase in total energy has an electrostatic origin and is almost one order of magnitude higher for $\mathrm{Ge}_{4}(\mathrm{GaAs})_{2}$ than the formation energy of strained $\mathrm{Si}_{4} \mathrm{Ge}_{4}$. This increase of the formation energy of 
the junction forces an atomic reconstruction at the interface. Therefore, the fundamental criterion which fixes the position of the Fermi level is the minimization of total energy[31]. The charge neutrality concept is then a natural consequence of the former.

\section{Alkali Metal-Semiconductor Interface}

Alkali atoms are usually characterized by a single valence electron in an orbit of large atomic radius. In the metallic state, the structure is open, and the charge density is featureless and low as compared to other divalent and trivalent close packed metals. In the band structure language the conduction band can be described by a nearly free electron picture leading to a spherical Fermi surface with a small value of the Fermi momentum. Interesting coverage dependent features of the alkali adsorption on metal surfaces have appeared from the work of Mac Rae et al.[53]. At the initial stage of cesium adsorption on the W(001) surface the work function $\phi$ decreases rapidly, and $\mathrm{Cs}$ atoms form a $2 \times 2$ structure with a Cs-Cs nearest neighbor distance significantly smaller than that in the Cs metal. Further Cs deposition (above the monolayer coverage) yields a close packed hexagonal Cs layer with a nearest neighbor distance comparable to that of the bulk metal. At this coverage the work function passes through a minimum, and a loss peak grows in intensity. Subsequent studies have shown that these coverage dependent features are common to other metal surfaces.

It is also well known that the deposition of alkali metals (AM) at sub-monolayer coverages on semiconductors leads to a significant reduction of the work function. In particular, if the work function is lowered to such an extent that the vacuum level falls below the bulk conduction band then the system is said to be driven in the negative electron affinity state (NEA). It was first reported[54] back in 1970 and confirmed[55] subsequently that the $\mathrm{Si}(001)$ surface can be activated to NEA by adsorption of AM (and subsequent addition of oxygen). These systems have important technological applications as high efficiency emitters and have since been actively pursued[56]. Furthermore, AM-assisted oxidation of semiconductors has potential applications in microelectronics industry. More recently, a school of thought emerged that since AM when adsorbed on semiconductors do tot react or interdiffuse with the substrate, they may serve to elucidate fundamental aspects of metallization[5] so vital to the SB problem. Thus there is a flurry of activity both experimentally and theoretically. Significant new results have emerged along with some controversial findings which have made the study of interaction of AM with semiconductors 
an area of active research. The main point of interest has been whether the alkali-semiconductor interaction and resulting modifications are different from the alkali metal-metal interactions. We first summarize some of the outstanding issues on the subject.

\section{A. Work function}

One of the early indications that the bonding of AM to semiconductors may be different from their bonding to other metals came from the work function $(\phi)$ measurements[57-60]. In general, for AM adsorption on other metals $[61,62], \phi$ decreases almost linearly at low coverages, passes through a minimum and then rises to a saturation value which corresponds to $\phi$ of the bulk AM. The variation of $\phi$ with $\theta$ measured for typical metal and semiconductor substrates[63] is shown in Fig. 13. The important point to notice in the metal data is that the minimum of $\phi$ is rather pronounced and occurs at coverages much lower than $\theta=1$. For AM on semiconductors there are reports $[57,60]$ of the absence of the minimum in work function. The minimum of $\phi$ shown in Fig. 13(c-d) is not so pronounced. In any case, when a minimum is observed, it occurs at higher coverages $[58,59,64,65]$ than those typically reported for the AM-metal systems. The SCFpseudopotential calculations[66] for $\mathrm{K}$ adsorbed on $\mathrm{Si}(001)-(2 \times 1)$ at $\theta=1$ indicates a reduction of the work function, $(\Delta \phi=2.3 \mathrm{eV})$, which is in fair agreement with the experimental observations.

\section{B. Alkali coverage}

Another difference from metals is the observation that $\mathrm{AM}$ on $\mathrm{Si}(001)-(2 \times 1)$ lead to a saturated overlayer at low temperature. The value of the saturation coverage itself, however, is unsettled. Based on a strong $2 \times 1$ low-energy electron diffraction (LEED) pattern at saturation, several authors $[57,60,67,68]$ have inferred the saturation coverage for $\mathrm{K}$ on $\mathrm{Si}(001)-(2 \times 1)$ at room temperature to be $1 / 2$ a monolayer $(\mathrm{ML})$. Since a monolayer coverage has been defined differently in the literature, to avoid any confusion, we let $\theta$ be the number of alkali metal atoms per $\mathrm{Si}(001)$ $(2 \times 1)$ surface unit cell, thus keep a unique definition throughout this work. Accordingly 1 ML (or $\theta=2$ ) coverage corresponds to $6.78 \times 10^{14}$ adsorbates $/ \mathrm{cm}^{2}$ in our definition. Enta et al. [58], on the other hand, from their angular resolved ultra-violet photoemission spectroscopy (ARUPS) data have concluded that the saturation coverage is $1 \mathrm{ML}$. Abukawa and Kono[59] have also concluded, based on a kinematical analysis of the $\mathrm{x}$-ray photoelectron diffraction patterns of $\mathrm{K} 2 p$ core levels, that the saturation coverage is $1 \mathrm{ML}$. Oellig and Miranda[69] assert that the coverage can be much 

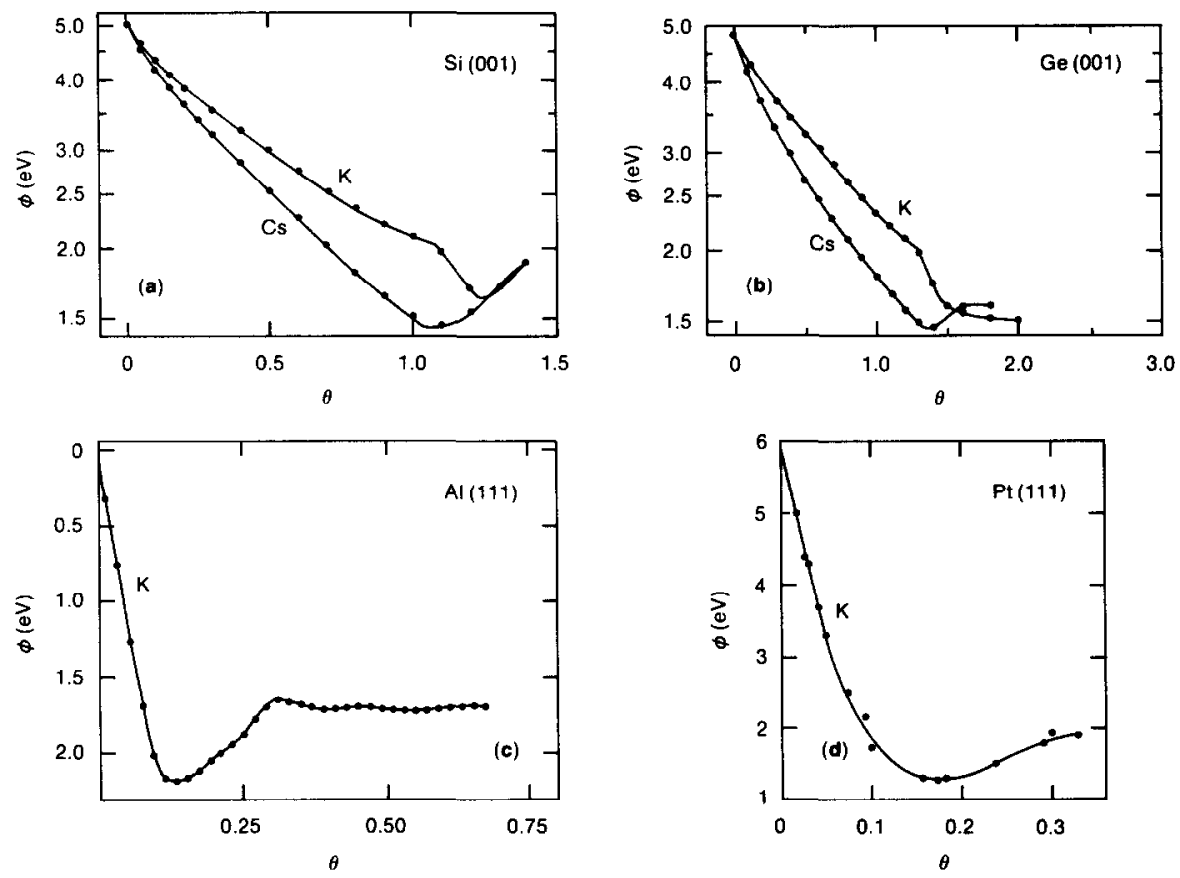

Fig. 13. Variation of the work function $\phi$ as a function of the alkali coverage on metal and semiconductor surfaces. (Reproduced from Ref. [63]).

higher at room temperature. In fact, based on the interpretation that their Si LMM Auger electron spectroscopy (AES) intensity data showing at least four breaks, they claim a layer-by-layer growth of $\mathrm{K}$ on $\mathrm{Si}(001)$. This claim has been challenged by Enta et al.[58] based on their own $\Delta \phi$ and AES data. The maximum reported coverage is problematic due perhaps to strong temperature dependence[70,60] of the coverage around room temperature. It has been reported[70] that around $300 \mathrm{~K}$, a slight variation in the actual temperature of the sample can result in a different AM coverage. For example at $273 \mathrm{~K}, 1 \mathrm{ML}$ may be stabilized while at $325 \mathrm{~K}$ the saturation coverage is in between 0.5 and $1 \mathrm{ML}$.

The situation has been clarified by the more recent detailed LEED, $\Delta \phi$ and AES measurements for Na-Si(001) by Glander and Webb[71]. They performed two types of experiments which they called dosing and equilibrium experiments. In the former type, which are the more usual kind of experiments, the observations are made as a function of time after turning on a constant atomic beam flux on a substrate held at a fixed temperature. These types of experiments may also be characterized as transient as opposed to equilibrium or steady state where coverage is changed by varying the crystal temperature for a fixed AM flux; the temperature of the sample is changed 
slowly enough to ensure that adsorbates are in a steady state (desorption and adsorption rates are equal). They conclude that for a wide range of effective pressures and temperatures the sticking coefficient changes abruptly from one to zero at the saturation coverage of $0.68 \mathrm{ML}$ of $\mathrm{Na}$. One can grow bulk $\mathrm{Na}$ in islands only at low temperatures and high pressures.

The LEED data on AM-Si(001) appears to have consensus at least as far as the dominant features are concerned. The clean $2 \times 1$ structure changes $[67,71]$ to a third order structure $(2 \times 3$ or $3 \times 2)$ and finally back to $2 \times 1$ structure at saturation coverage with spot intensities different from that for the clean surface. There are reports of $4 \times 1$ structure at $0.25 \mathrm{ML}$ in dosing experiments and occasionally $2 \times 3$ LEED patterns have not been observed. Glander and Webb[71] have also noted an incommensurate phase between $2 \times 3$ and $2 \times 1$ as a function of increasing coverage.

\section{Adsorption site}

The optimum adsorption site for $\mathrm{AM}$ on $\mathrm{Si}(001)-(2 \times 1)$ at various coverages are still being actively investigated[72-74]. These possible adsorption sites are shown in Fig. 14. In 1973 Levine[67] proposed for $\mathrm{Cs}$ adsorption on $\mathrm{Si}(001)$ that at low coverages this metal occupied a quasi-hexagonal hollow site $(\mathrm{H})$ above the rows of dimers as shown in Fig. 14(a). The H site offered a simple explanation for the NEA because oxygen atoms could submerge under the long bridge $(B)$ sites to cause additional (beyond that produced by AM) lowering of the work function required to achieve NEA $[67,75]$. Since then this adsorption site has been widely used for other adsorbates as well, in qualitative discussions of various properties of $\mathrm{AM}-\mathrm{Si}(001)$ interfaces. Most of the LEED data is consistent $[67,68,71]$ with this adsorption site as is the inverse photoemission data.

Scanning tunneling microscopy (STM) experiments have been performed recently[76-78] on various AM-semiconductor systems. These include $\mathrm{Cs}-\mathrm{GaAs}(110)$ and $\mathrm{K}, \mathrm{Li}$ on $\mathrm{Si}(001)-(2 \times 1)$. The STM data on Cs-GaAs(110) was interpreted[76] in terms of periodic one-dimensional Cs chain like structure. Hasegawa et al.[77] from their STM data have suggested that at low coverages, Li, $\mathrm{K}$ atoms are adsorbed in on top ( $\mathrm{C}$ ) site above one of the dimer forming Si atoms. But at about 0.1 ML coverage AM formed linear chains perpendicular to the Si dimer rows. STM work by Badt et al.[78] on $\mathrm{K}-\mathrm{Si}(001)-(2 \times 1)$ noted considerable disorder. Also absent were any long chains of $\mathrm{K}$ atoms.

Another experiment $[70,79]$ directly dealing with adsorption site of $\mathrm{K}$ on $\mathrm{Si}(001)$ is through titration with physisorbed Xe. From this it was concluded that the long bridge site B [see Fig. 13(a)] is preferentially filled over an $\mathrm{H}$ site at $1 / 2 \mathrm{ML}$ coverage. This experiment requires 

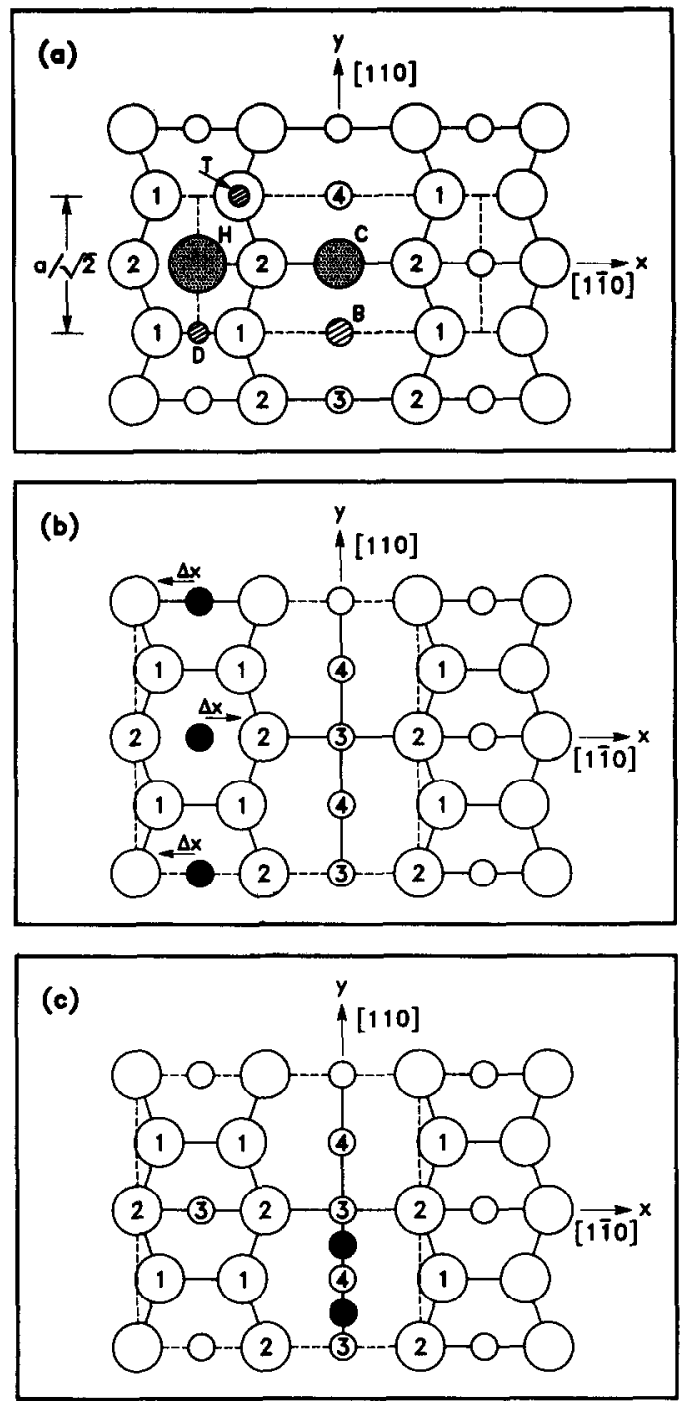

Fig. 14. Top views describing the positions of alkali metals (AM) on $\mathrm{Si}(001)-(2 \times 1)$. Filled and empty circles denote $\mathrm{AM}$ and $\mathrm{Si}$ atoms, respectively. Numerals in the circles indicate $\mathrm{Si}$ atomic layers. (a) $\mathrm{H}, \mathrm{B}$, and $\mathrm{C}$ sites have been labeled in the $2 \times 1$ unit cell shown by dotted lines. At $1 / 2 \mathrm{ML}$ coverage $(\theta=1)$ only one of these sites is occupied. At $1 \mathrm{ML}(\theta=2)(\mathrm{H}-\mathrm{B})$ or $(\mathrm{H}-\mathrm{C})$ are simultaneously occupied. (b) The $2 \times 2$ unit cell shown by dotted lines used to study $\Delta x$ Peierls' distorted structure of $\Lambda \mathrm{M}$ at $\theta=1$. (c) The $2 \times 2$ unit cell shown by dotted lines used to study pairing of $A M$ atoms ( $\Delta y$ distortion) of $A M$ at $\theta=1$. (Reproduced from Ref. [88])

a precise knowledge of the Xe thermal desorption spectra and correlation of various peaks with the surface adsorption sites. Unfortunately, this is not known in an unambiguous fashion. In addition 
to the site there is also a lack of agreement on the value of the K-Si bond length. Values in the range of 2.6 - 3.5 $\AA$ have been published[70,79]. There is only one direct measurement[80] for K-Si bond length which gave a value of $3.14 \pm 0.10 \AA$. This has been derived from surface extended $x$-ray absorption fine-structure (SEXAFS) data[80] but is not site specific.

The conclusions drawn from various calculations[66,72-74,81-85] are also somewhat diverse. Early theoretical work[81] did not carry out total energy calculations. They accepted the $\mathrm{H}$ site as a given but varied the vertical height of $\mathrm{AM}$ overlayer to obtain agreement with some measured property. The K-Si bond length, $d=3.52 \AA$ was inferred. Subsequently, results were presented[82] for several values of $d$.

The first total energy calculations for the $\mathrm{H}$ site were performed by Ciraci and Batra[66] using the pseudopotential method. For $\theta=1$, they obtained an optimized value of $d(\mathrm{~K}-\mathrm{Si})=2.6 \AA$ at the $\mathrm{H}$ site with a binding energy $E_{b} \simeq 3 \mathrm{eV} /$ per AM. The $\mathrm{K}$-Si interatomic distance might be underestimated in these calculations due to the particular form of the ionic pseudopotential and possibly due also to the form of the exchange-correlation potential. A calculation based on the pseudofunction method[83] obtained a $d(\mathrm{~K}-\mathrm{Si})$ of $3.32 \AA$ at the $\mathrm{H}$ site. A small cluster model calculation[84] based on the SCF-Hartree-Fock method again performed for the $\mathrm{H}$ site showed that the minimum in total energy as a function of vertical height of $K$ was rather shallow (see Fig. 15). This was offered as an explanation for a range of published $d$ values. The authors stated an optimum height of $d(\mathrm{~K}-\mathrm{Si})=3.52 \AA$. Although this value of $d$ is even larger than the sum of covalent radii $(3.3 \AA)$ the bond was shown to be highly ionic. The authors therefore cautioned against deducing the nature of bond from value of the bond length[84].

The relative stability of various sites ( $\mathrm{H}, \mathrm{B}, \mathrm{C}$-sites) was first examined by Batra[72] for Na$\mathrm{Si}(001)-(2 \times 1)$. The basic conclusion was that the $\mathrm{H}$ and $\mathrm{B}$ sites are equally favored as far as the adsorption energy is concerned. Ling et al.[73] have recently carried out extensive cluster model calculations for the K-Si system within the framework of local density total energy formalism. In agreement with earlier theoretical results[66], they found strong ionic bonding at both the competing $\mathrm{H}$ and $\mathrm{B}$ sites. For example, at the $\mathrm{H}$ site they obtained $\Delta Q \sim 0.63$ e from $\mathrm{K}$ to $\mathrm{Si}$ and a binding energy of about $3 \mathrm{eV}$. Their computed value of $d(\mathrm{~K}-\mathrm{Si}) \sim 3.2 \AA$ is also close to the sum of atomic radii but again the bond has large ionicity. They noted that the adsorption at the $\mathrm{H}$ site is actually unstable to a zig-zag distortion of $\mathrm{K}$ atoms and concluded that the $\mathrm{B}$ site is more stable than the $\mathrm{H}$ site. It should be noted that reliable conclusion at the $\mathrm{B}$ site demands extreme numerical accuracy in the calculation. For example, the calculated change in energy[73] was only $0.002 \mathrm{eV}$ when the vertical height of the $\mathrm{K}$ was changed by $0.1 \mathrm{a}$.u. around the equilibrium 


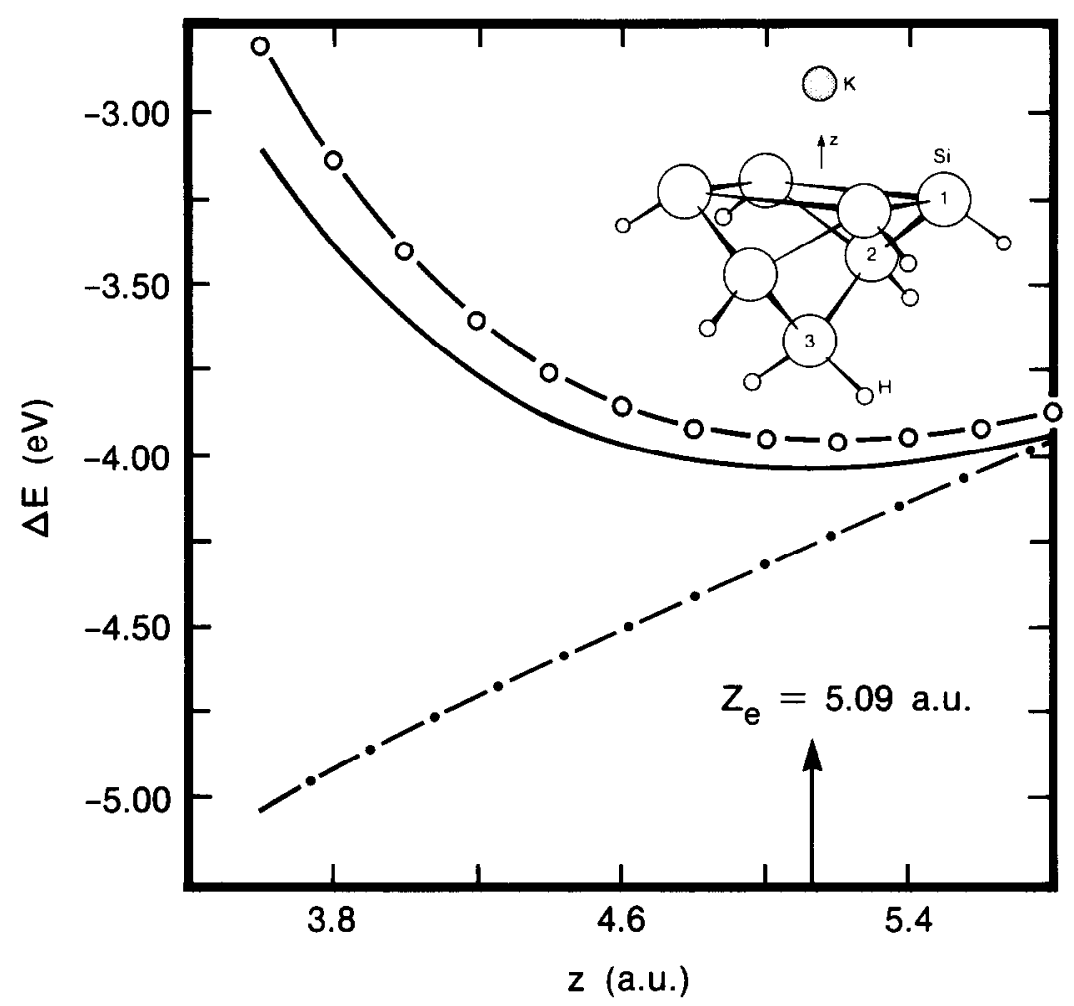

Fig. 15. Potential energy curves calculated by SCF-Hartree-Fock method. Upper curve with circles is for $\mathrm{Si}_{7} \mathrm{H}_{8}^{-}$and $\mathrm{K}^{+}$. Curve with continuous line correspond to $\mathrm{Si}_{7} \mathrm{H}_{8} \mathrm{~K}$ described in the inset, and curve with dots obtained from $\mathrm{Si}_{7} \mathrm{H}_{8}^{-}$and a point charge of $q=+1$. $z$ is the distance of $\mathrm{K}$ or the point charge from the Si surface. (Reproduced from Ref. [84]).

position. They also examined other sites and obtained total energies (in the order of decreasing stability) $E(B)<E(C)<E(H) \simeq E(D)$. The corresponding $d(\mathrm{~K}-\mathrm{Si})$ calculated by them in the same order are 3.2, 3.5, 3.2, and 3.1 $\AA$.

More recently, a slab calculation[74] based on SCF-Hartree-Fock-INDO scheme has also examined the relative stability of the four sites and obtained the same filling order: $\mathrm{B}, \mathrm{C}, \mathrm{H}$, and $\mathrm{D}$. at $1 / 2$ ML coverage $(\theta=1)$. The calculated $d(\mathrm{~K}-\mathrm{Si})$ values, however, are $2.65,3.34,2.69$, and $2.52 \AA$ respectively. Another key variance from the cluster model results[73] is in the spread of total energy, i. e., the difference in total energy between the most and the least stable site. Whereas Ling et al.[73] report a spread of about $0.75 \mathrm{eV}$, the value by Ramirez[74] is about $5 \mathrm{eV}$.

At saturation coverages, Abukawa and Kono[59] have proposed the existence of a $\mathrm{K}$ double layer. These results were based on a kinematical analysis of the x-ray photoelectron diffraction patterns 


\section{I.P. Batra et al.}

e levels. They found that placing $\mathrm{K}$ atoms in (H-C) sites simultaneously (see Fig. 14) ed configuration successfully explained their observations. The dilayer corresponds to of $1 \mathrm{ML}$. This model has been supported by the calculations of Tsukada et al.[82] sased on pseudopotential calculations, had proposed the (H-B) model. Ramirez[74] has that the (H-B) model, is $1.1 \mathrm{eV} /$ cell more stable than the (H-C) model.

substrate model would be a reasonable approximation if it were not for the fact that geometry of the $\mathrm{Si}(001)-(2 \times 1)$ surface is still not completely known[86,87]. There is a ement $[86,87]$ as to whether the Si dimers are symmetric and/or buckled. One does not expect a significant lattice rearrangement due to the presence of AM. But since the zometry itself is uncertain, the optimization of the entire system becomes essential. The it in considering the relaxation[88] of the substrate concomitant with the adsorption of swed that the optimization leads to conclusion which are significant for site selectivity. shieved by performing a number of total energy, atomic force and electronic structure ; for $\mathrm{Na}, \mathrm{K}$ adsorption on $\mathrm{Si}(001)$ at two coverages, $\theta=1$ and 2 , on various surface $r$ optimizing geometries in the presence of AM overlayers placed at various adsorption it results related to the lattice relaxation (full structural optimization) will be given in F.

$\underline{\text { ure at } \theta=1(1 / 2 \text { a } M L \text { coverage })}$

hensive optimization of some binding structures at $\theta=1(1 / 2 \mathrm{ML})$ was carried out for ing the SCF-total energy calculations[72,88]. The results are summarized in Table 1 sites. The initial optimization consisted of only moving the AM overlayer along the while the substrate atoms were held fixed at the positions given by the AbrahamAB) symmetric dimer model. Therefore, such a optimization is limited. An important te is that both $\mathrm{H}$ and $\mathrm{B}$ adsorption sites have nearly the same adsorption energies. appears to be slightly more favorable $(\sim 0.01 \mathrm{eV} /$ cell $)$ than the $\mathrm{H}$ site when the energy reased to $6.5 \mathrm{Ry}$. However, the difference is too small and does not warrant a clear 'een the $\mathrm{H}$ and $\mathrm{B}$ sites. At this point a judicious conclusion would be that at room $\mathrm{e}$ both $\mathrm{H}$ and $\mathrm{B}$ sites shall be occupied, governed only by the kinetics considerations. : able to give a more definitive conclusion in subsection $\mathrm{F}$ upon including the lattice The occupancy of $\mathrm{C}$ site is found to be somewhat unfavorable at 1/2 ML but the $\mathrm{D}$ can be clearly ruled out. Also from the last two columns of Table 1 it is clear that 
reverting to an ideal surface at this coverage is energetically unfavorable. Although the bonding on the ideal surface is actually more favorable but the loss in energy due to dimer breaking $(\sim 1.6$ $\mathrm{eV}$ ) is not offset by this additional bonding.

TABLE 1. Calculated relative energies (in $\mathrm{eV}$ ) measured with respect to $H$ site for various adsorption sites for $\mathrm{Na}$ at $\theta=1$ on $\mathrm{Si}(001)-(2 \times 1)$ and ideal (I) $\mathrm{Si}(001)$ surfaces. The optimized vertical heights, $h$, and nearest neighbor $\mathrm{Na}$-Si interatomic distances $d$ are given in $\AA$. Positive values for $\Delta E$ correspond to energetically less favorable configurations. The Bloch states were represented by a basis set of $\sim 550$ plane waves corresponding to $|\vec{k}+\vec{G}|^{2}<4.5$ Ry. The numbers in parenthesis are for calculations performed with $\sim 1000$ plane waves, $|\vec{k}+\vec{G}|^{2}<6.5$ Ry. (Reproduced from Ref. [88]).

\begin{tabular}{|cccccccc|}
\hline Sites & $\mathrm{H}$ & $\mathrm{B}$ & $\mathrm{C}$ & $\mathrm{D}$ & $\mathrm{T}$ & $\mathrm{I}(\mathrm{H})$ & $\mathrm{I}(\mathrm{B})$ \\
\hline$\Delta E$ & 0.0 & $0.03(-0.01)$ & $0.14(0.14)$ & 0.60 & 0.78 & 0.89 & 1.32 \\
& & & & & & & \\
$h$ & $1.27(1.32)$ & $-0.16(-0.29)$ & $0.64(0.58)$ & 2.27 & 2.49 & 0.79 & 1.80 \\
$d$ & $2.60(2.62)$ & $2.64(2.66)$ & $2.83(2.79)$ & 2.57 & 2.49 & 2.83 & 2.63 \\
\hline
\end{tabular}

In Fig. 16 we have reproduced[88] plots of total energy vs vertical height of the AM overlayer at various sites at $\theta=1$ for $\mathrm{Na-Si}$. Similar results were obtained for the K-Si system. All energies are referenced with respect to energy of the most stable configuration at the $\mathrm{H}$ site and are computed using a 4.5 Ry energy cutoff. The shape of the energy curve around the minimum offers a hint that the $\mathrm{H}$ and $\mathrm{B}$ sites would be differentially affected upon lattice relaxation. The energy sequence (in the order of decreasing stability), $E(H) \simeq E(B)<E(C)<E(D)<E(T)$, convinces us that the $\mathrm{D}$ and $\mathrm{T}$ sites can be safely ruled out at this coverage. It thus emerges that the $\mathrm{H}$ and $\mathrm{B}$ sites are competitive adsorption sites with the $\mathrm{C}$ site being a close third at $\theta=1$.

\section{E. Peierls' distortions}

Earlier, it was pointed out that $\mathrm{K}$ adsorbed at the $\mathrm{H}$ site can be viewed as quasi 1D metal. Moreover, the anisotropic and positive dispersion obtained from the angle-resolved electron-energy loss spectroscopy (EELS) was attributed to the 1D plasmon[89] associated with the alkali metal chain. Accordingly one expects some kind of Peierls' distortion which should lower the total energy of the alkali overlayer. Such a distortion may also be decisive for the adsorption site. In 


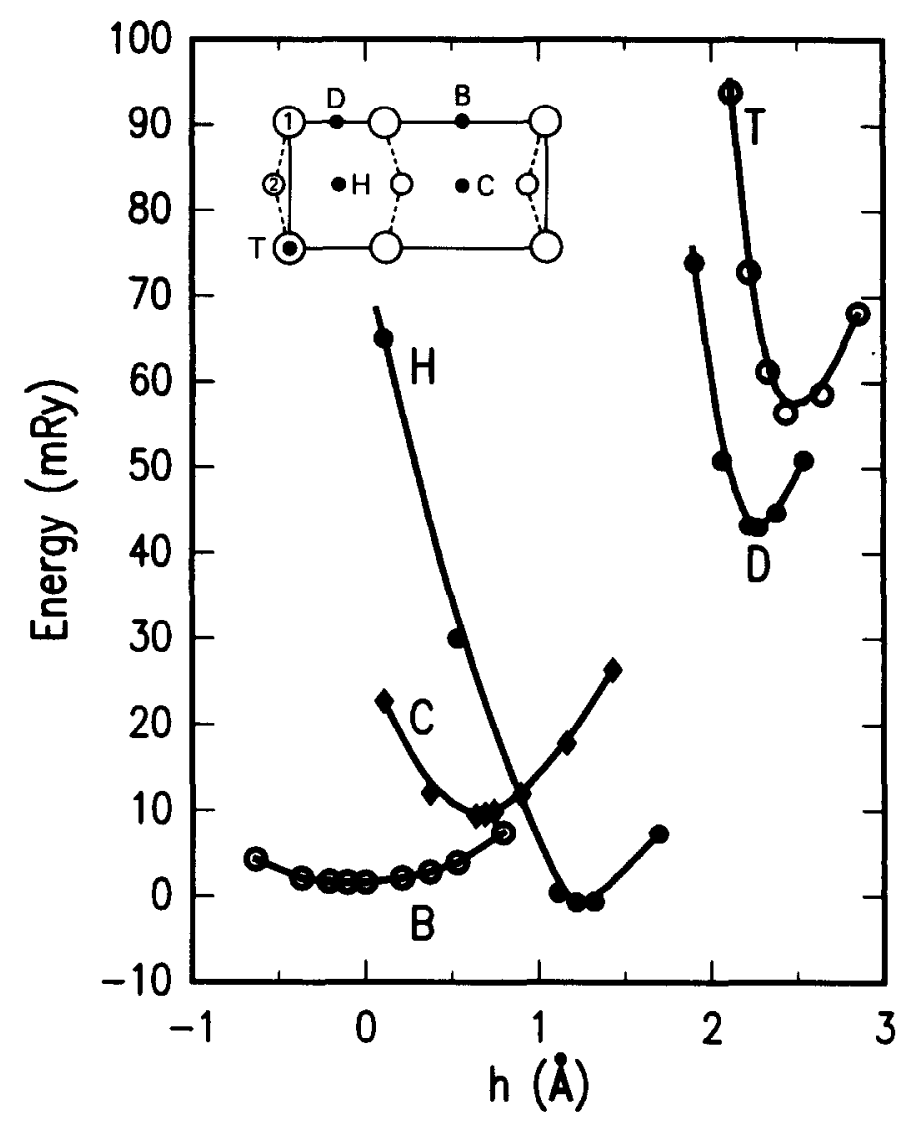

Fig. 16. Total energy as a function of the vertical height, $h$, of the alkali metal overlayer above the $\mathrm{Si}(001)-(2 \times 1)$ surface at various adsorption sites shown in the inset. Substrate atoms were held in fixed positions given by the Abraham-Batra[87] symmetric dimer model. All energies are referenced with respect to the most stable H site. (Reproduced from Ref. [88]).

fact, recently Ling et al.[73] claimed that the adsorption at the $\mathrm{H}$ site is unstable to a zig-zag $\Delta x$ distortion of $\mathrm{K}$ atoms shown in Fig. 14(b). They used the instability of the $\mathrm{H}$ site towards such a distortion as an indication that the optimum adsorption site may in fact lie elsewhere. They thus concluded[73] that the long bridge site is more stable than the $\mathrm{H}$ site.

More recently, Batra[88] computed the total energy at the $H$ site as a function of $\Delta x$ within the slab model having the $2 \times 2$ periodic cell. His SCF-pseudopotential results for $\mathrm{Na}$ overlayer held at a fixed height $(\sim 1.2 \AA)$ above the $\operatorname{Si}(001)-(2 \times 1)$ surface do not show any instability due to the $\Delta x$ distortion. Results were similar for the K-Si system as inferred from calculations done using only a few $\Delta x$ values. The source of an important disagreement between SCF-pseudopotential and cluster calculations was first sought in the different structural parameters used in these calculations. 
Nevertheless, SCF-pseudopotential calculations carried out with different structural parameters (for example taking K-Si bond at the empirical value, which is close to the value of Ling et al.[73] and to the SEXAFS result[80]) and also relaxing the substrate at each time were not of any help in favor of the zig-zag chain. In all cases, the total energy of the system increased upon a zig-zag distortion of the chain by any $\Delta x$. The total energy of the unsupported AM layer (corresponding to infinite $d$ ) in registry with the $\mathrm{H}$ site adsorption increased in energy by $4.5 \mathrm{mRy} / \mathrm{AM}$ atom upon a zig-zag deformation of $\Delta x=0.5 \AA$.

It is then important to note that the $\Delta x$ motion of AM atoms does not open any energy gap at the zone edge. This follows from the fact that the space group for the $\Delta x$ distorted structure is nonsymmorphic. Consequently, all bands have to be doubly degenerate at one zone boundary. The Fermi level passes through the degeneracy point at the zone edge and the system is intrinsically metallic. Thus the energy lowering mechanism due to a gap opening at the zone edge[73] is not operative[88] for the $\Delta x$ distortion. Clearly the site energies remain degenerate upon $\Delta x$ distortion. The change in total energy arises from the change in band width. On the other hand a small $\Delta y= \pm 0.25 \AA$ distortion lowered the total energy by $1.1 \mathrm{mRy}$. The $\Delta y$ distortion is a dimer forming distortion which breaks the degeneracy at the zone edge and the energy is lowered due to the Peierls' mechanism. For the AM overlayer adsorbed at the $\mathrm{H}$ sites any major $\Delta y$ distortion is precluded due to the presence of surface $\mathrm{Si}$ atoms.

The energy lowering upon $\Delta x$ distortion found by Ling et al.[73] may have its origin in some cluster artifacts. it is also surprising that their energy continues to decrease monotonically even for a large $\Delta x$ which places the $\mathrm{K}$ atom at the edge of the cluster. It is possible to lower the total energy upon bond optimization without opening a gap at $E_{F}$. The lowering in energy is brought about by bond optimization because both the distorted and undistorted structures are metallic[88,90]. It is possible that the absence of a minimum in the cluster calculations is also a cluster artifact.

The absence of $\Delta x$ distortion for AM in the SCF-pseudopotential calculations is due to resultant unfavorable bonding. Since the alkali metal is in a more or less ionized state, the $\Delta x$ distortion amounts to essentially moving bare AM ions towards $\mathrm{Si}$. This tends to raise the energy since the $\Delta x$ distortion does not open a gap at $E_{F}$.

The $\Delta y$ type of Peierls' distorted structure at the B sites shown in Fig. 14(c) offers another adsorption geometry at $\theta=1$. This corresponds to the dimerization of $\mathrm{Na}$ at the $\mathrm{B}$ sites. Although such dimerization has been observed[91] for Ga adsorption on $\mathrm{Si}(001)$, for AM Batra[88] found such a dimerization is energetically unfavorable. The origin of this is traced to the fact that AM atoms 
are in a more or less ionized state on the surface. Bringing the ionized atoms towards each other does not create any bonding interaction and hence the energy is raised. Thus the AM dimerization ( $\Delta y$ distortion) at the $\mathrm{B}$ sites is also ruled out.

In closing this subsection, we emphasize that in the periodic slab model calculations the $\Delta x$ distortion of the $\mathrm{H}$ sites and $\Delta y$ distortion of the $\mathrm{B}$ sites are energetically unfavorable. Thus both the $\mathrm{H}$ and $\mathrm{B}$ sites are competitive overlayer adsorption sites in the absence of the substrate relaxation which we now address.

\section{F. Fully optimized structure}

It emerges that the $\mathrm{H}$ and $\mathrm{B}$ sites are important sites at $\theta=1$ and are also consistent with the observed $2 \times 1$ LEED patterns. Now we consider the substrate relaxation. Complete relaxation calculations were carried out for $\mathrm{Na}-\mathrm{Si}$ system[88] and one expects similar relaxations for $\mathrm{K}-\mathrm{Si}$. So far the substrate atoms were held in fixed positions given by the AB symmetric dimer model[87]. For the calculational parameters at hand forces $\leq 0.05 \times 10^{-8} \mathrm{~N}$ are to be considered small. The $y$-components of forces were always negligibly small. However, for $\mathrm{Na}$ in the optimized position, significant atomic forces were found on the first and third layer $\mathrm{Si}$ atoms, which are apparently the internal forces in the $\mathrm{AB}$ dimer model. The direction of forces on the top layer $\mathrm{Si}$ atoms indicates that the dimer bond wants to shrink somewhat and the layer as a whole wants to relax inwards. The forces on the substrate atoms implies the importance of the substrate relaxation.

Starting from the AB symmetric dimer model[87], which is free of internal forces, the relaxation of the substrate upon AM adsorption has been considered. By moving atoms along the force direction the optimized structure given in Table 2 was obtained. The major relaxation introduced in the lattice (relative to the structure of the substrate) upon the adsorption of $\mathrm{Na}$ at the $\mathrm{H}$ sites is to increase the Si dimer bond length towards bulk value by 0.2 a.u. This is still 0.06 a.u. smaller than the equilibrium bulk value. The direction of relaxations towards bulk values are physically reasonable because $\mathrm{AM}$ overlayer supplies some charge to the dangling bonds. To the dangling bonds it appears as though there is another layer of Si present and there is an attempt to continue the $\mathrm{Si}$ lattice. Hence the relaxation is in the direction of the bulk.

Similar structure optimizations carried out also for the $\mathrm{B}$ and $\mathrm{C}$ sites. The most significant finding is the energy sequence: $E(\mathrm{H})<E(\mathrm{~B})<E(\mathrm{C})$. The $\mathrm{B}$ and $\mathrm{C}$ sites are less stable than the $\mathrm{H}$ site by 0.15 and $0.25 \mathrm{eV}$ respectively. These values changed to 0.12 and $0.22 \mathrm{eV}$ upon increasing the energy cutoff to $7.5 \mathrm{Ry}$. The distinction between $\mathrm{H}$ and $\mathrm{B}$ sites became sharper, unlike the 
TABLE 2. Optimized atomic coordinates and the residual forces acting on various atoms. Forces (in units of $10^{-8} \mathrm{~N}$ ) for $\mathrm{Si}$ atoms in the top four layers and on $\mathrm{Na}$ are given. Forces were computed using the $2 \times 2$ cell. The Bloch states were represented by a basis set of 730 plane waves corresponding to $|\vec{k}+\vec{G}|^{2}<5.5 \mathrm{Ry}$ and $9 \mathrm{k}$-points in the Brillouin zone. No significant $y$ forces $\left(F_{y}=0\right)$ were detected. (Reproduced from Ref.[88]).

\begin{tabular}{|cccccc|}
\hline $\mathrm{N}$ & $\mathrm{x}$ & $\mathrm{y}$ & $\mathrm{z}$ & $F_{x}$ & $F_{z}$ \\
\hline $\mathrm{Na}$ & 0.0 & 3.63 & 1.85 & 0.0 & 0.005 \\
1 & -2.19 & 0.0 & -0.62 & 0.012 & -0.032 \\
$1^{\prime}$ & 2.19 & 0.0 & -0.62 & -0.012 & -0.032 \\
2 & -3.40 & 3.63 & -2.73 & 0.028 & -0.026 \\
$2^{\prime}$ & 3.40 & 3.63 & -2.73 & -0.028 & -0.026 \\
3 & 0.0 & 3.63 & -5.51 & 0.0 & 0.004 \\
$3^{\prime}$ & 7.26 & 3.63 & -5.05 & 0.0 & 0.049 \\
4 & 0.0 & 0.0 & -7.89 & 0.0 & -0.002 \\
$4^{\prime}$ & 7.26 & 0.0 & -7.63 & 0.0 & -0.014 \\
\hline
\end{tabular}

results shown in Fig. 16 for the fixed lattice given by AB. This has been brought about by the preferential lowering of the energy at the $\mathrm{H}$ site relative to the $\mathrm{B}$ site upon lattice relaxation. The $\mathrm{C}$ site also benefits more than the B site but since it had higher energy to start with it continues to be less stable. A physical argument in favor of $\mathrm{H}$ site over $\mathrm{B}$ and $\mathrm{C}$ sites can be constructed. Since AM atoms are fairly electropositive they can be well screened from each other at the $\mathrm{H}$ sites by the intervening electronic charge in the dangling bonds. Such a screening is much less effective at the $\mathrm{B}$ and $\mathrm{C}$ sites. Our computed $d(\mathrm{Na}-\mathrm{Si})$ are $2.60,2.75$ and $2.83 \AA$ at $\mathrm{H}, \mathrm{B}$ and $\mathrm{C}$ sites respectively. These values have not changed much upon optimization.

By sampling the charge density at the $32-\mathrm{k}$ points in the Brillouin zone and using an extended basis set corresponding to an energy cutoff 7.5 Ry the structure of adsorbate substrate combined system was optimized[88]. These results are given in Table 3. An extensive LEED calculation[70] 
has concluded that at $1 / 2$ a ML coverage best agreement with data is obtained for Na overlayer adsorbed at the $\mathrm{H}$ sites at a vertical distance of $1.85 \AA$. In fact, the two structures with relatively low Pendry $R$-factor (averaged over several beams) values of 0.25 and 0.33 both involve adsorption at the $\mathrm{H}$ sites but with different vertical heights of the Na overlayer of 1.85 and $1.25 \AA$ respectively. Based on the $\mathrm{R}$-factor value the structural model with $h=1.85 \AA$ was selected as the optimum. The distance obtained from the SCF-pseudopotential calculations in Table 3 at the $\mathrm{H}$ site is somewhat smaller $(\sim 1.3 \AA)$.

TABLE 3. Optimized atomic coordinates (in a.u.) for $\mathrm{Na}$ at $\mathrm{H}$ and $\mathrm{B}$ sites and the corresponding top two $\mathrm{Si}$ layers in the $2 \times 1$ cell. These calculations were performed with $7.5 \mathrm{Ry}$ cutoff and $32 \mathrm{k}$-points in the Brillouin zone. No significant y-forces $\left(F_{y}=0\right)$ were detected.Atomic coordinates for the other layers are those given in Table 2. (Reproduced from ref. [88]).

\begin{tabular}{|ccccccc|}
\hline $\mathrm{N}$ & $\mathrm{x}$ & $\mathrm{y}$ & $\mathrm{z}$ & $F_{x}$ & $F_{z}$ \\
\hline $\mathrm{Na}(\mathrm{H})$ & 0.0 & 3.63 & 1.85 & 0.0 & 0.008 \\
1 & -2.19 & 0.0 & -0.65 & -0.001 & -0.019 \\
$1^{\prime}$ & 2.19 & 0.0 & -0.65 & 0.001 & -0.019 \\
2 & -3.40 & 3.63 & -2.73 & -0.010 & -0.025 \\
$2^{\prime}$ & 3.40 & 3.63 & -2.73 & 0.010 & -0.025 \\
\hline $\mathrm{Na}(\mathrm{B})$ & 7.26 & 0.0 & 0.40 & 0.0 & -0.032 \\
1 & -2.19 & 0.0 & -0.50 & -0.024 & 0.043 \\
$1^{\prime}$ & 2.19 & 0.0 & -0.50 & 0.024 & 0.043 \\
2 & -3.40 & 3.63 & -2.73 & 0.019 & 0.005 \\
$2^{\prime}$ & 3.40 & 3.63 & -2.73 & -0.019 & 0.005 \\
\hline
\end{tabular}

The $B$ adsorption site has been favored by the calculations of Ling et al.[73] and Ramirez[74] with K-Si bond lengths of $3.2 \AA$ and $2.65 \AA$ respectively. Although, Batra[88] found B site to be somewhat less stable for $\mathrm{Na}-\mathrm{Si}, d(\mathrm{Na}-\mathrm{Si})$ lies in this range and is $2.75 \AA$. All these bond length 
values imply a shorter vertical height of AM overlayer above the Si surface relative to the $\mathrm{H}$ site adsorption. In other words, a similar value of the bond length at $\mathrm{H}$ site shall place the AM overlayer at a higher vertical distance. It appears to us that these short vertical heights at $B$ sites are not likely to produce sufficient change in the normal dipole moment. Thus the large work function lowering which is often observed at semiconductor surfaces is difficult to explain[92] by the $\mathrm{B}$ site adsorption. Furthermorc, if $\mathrm{AM}$ are adsorbed on the $\mathrm{B}$ sites, one has yet to explain how $\mathrm{Si}(001)$ is activated to NEA since $\mathrm{B}$ sites are not available for oxygen adsorption. Recall that the NEA has always been explained[58,59,67] on the basis of the $\mathrm{H}$ site occupancy by AM. Another independent pseudopotential calculation[92] finds $\mathrm{H}$ and $\mathrm{B}$ sites to be competitive in total energy. Their calculated $\Delta \phi$ value leads to selecting $\mathrm{H}$ site in preference to $\mathrm{B}$ site. Freeman et al.[93] have recently completed calculations where substrate relaxation has been taken into account. They now agree with findings of Batra[88] that the $\mathrm{H}$ and $\mathrm{B}$ sites are difficult to discriminate for K-Si system based on total energy calculations. However, for $\mathrm{Na}-\mathrm{Si}$, in agreement with Batra[88], they favor[93] $\mathrm{H}$ site occupancy.

A recent STM data has been interpieted[77] in terms of adsorption at the T site at very low ( $\lesssim$ $0.1 \mathrm{ML}$ ) AM coverages. The lowest coverage used by Batra[88] is $1 / 2$ a $\mathrm{ML}$ and as such a direct comparison is not possible. However, some significant conclusions can be drawn by examining the calculated Hellmann-Feynman forces. The forces on various atoms in the optimized (with respect to the vertical height $h$ of the AM overlayer) structure at the T site (see Fig. 16) on a symmetric dimer model showed an asymmetric pattern. The dimer forming surface $\mathrm{Si}$ atom in the $2 \times 1$ cell, which did not have $\mathrm{Na}$ atom located above it, felt a large force of $0.2 \times 10^{-8} \mathrm{~N}$ along the $-z$ direction suggesting an asymmetric buckling of the surface. By placing the AM overlayer above the $\mathrm{T}$ site on the Yin-Cohen[86] asymmetric $\operatorname{Si}(001)-(2 \times 1)$ (as modified by Batra[87]) substrate, the total energy is lowered by about $14 \mathrm{mRy}$ with respect to the value shown in Fig. 16. Another $15 \mathrm{mRy}$ stabilization was achieved upon including complete substrate relaxation (most of which was due to stretching the surface dimer bond towards the bulk value of about 4.4 a.u.). Thus nearly $30 \mathrm{mRy}$ has been gained with respect to the value shown for the $\mathrm{T}$ site in Fig. 16. But it is still less stable than the $\mathrm{H}$ and $\mathrm{B}$ sites (even in the absence of substrate relaxation) shown in Fig. 16. Thus it is clear that at $1 / 2$ a ML, the $\mathrm{T}$ site is not the most stable site. However as proposed by Hasegawa et al.[77], an asymmetric buckling is definitely stabilized if and when the $\mathrm{T}$ site is occupied.

According to Ciraci and Batra[66,72] an AM overlayer partly fills the dangling bond band leading to a metallic state. Since STM is sensitive to charge density at $E_{F}$, it is conceivable that the surface dangling bonds are being imaged by the experiment[77]. This might give the impression of the $\mathrm{T}$ 
site occupancy. However, if an $\mathrm{H}$ site were to be occupied at the lowest coverage one $\mathrm{s}$ four surrounding $\mathrm{Si}$ atoms. Since only one site is imaged by the experiment one can speci adsorption site at the low coverage is evidently different from what is found at $1 / 2$ a ML If at low coverage the $\mathrm{T}$ site is occupied, then above calculation also supports the stabil the asymmetric dimerization postulated by Hasegawa et al.[77]. Coverage dependent ch possible for $A M$ because at $1 / 3 \mathrm{ML}$ coverage either a mixed site model or a major recon of the lattice has to be postulated[71] to explain the LEED data. This is discussed at subsection $\mathrm{H}$.

\section{G. Structure at $\theta=2$ coverage}

A plausible structure $[72,74]$ at $\theta=2(1 \mathrm{ML})$ involves occupying the $\mathrm{H}$ and $\mathrm{B}$ sites simult An alternative, involves occupying the $H$ and $C$ sites simultaneously $[58,59,92]$. Sim occupation of $\mathrm{B}$ and $\mathrm{C}$ sites can be ruled out on physical grounds. For a fixed lattic the (H-C) structure was found[72] to be less favorable by about $0.1 \mathrm{eV}$ per unit cell.

When one allows lattice relaxation[88] the (H-B) and the (H-C) described in Table 4 ha identical total energies. Thus, based on the total energy criteria above, the two struc indistinguishable. The AM overlayer attempts to lift the $2 \times 1$ reconstruction. This is evic the Si dimer bond length which is being stretched beyond its value at the surface ( $4.2 \mathrm{a} . \mathrm{u}$ as the bulk value (4.4 a.u.). The bond stretching is a natural consequence of $\pi^{*}$-band or The stretch offers a depolarization mechanism as more charge is transferred from AM to bonds at $\theta=2$. The AM overlayer does not succeed in fully lifting the reconstruction it is found[72] that reverting to an ideal surface is energetically unfavorable (by $\geq 0.6$ monolayer AM coverage. For comparison, the $2 \times 1$ reconstruction is lifted[90] by $\mathrm{Al}$ at about $1 / 2$ a ML coverage. Both the (H-B) and (H-C) structures used by Batra[90] are 1 with $\Delta h \simeq 0.7-0.9 \AA$. A puckered structure at monolayer coverage of $\mathrm{K}$ on $\mathrm{Si}(001)-($ : been proposed[59] with $\Delta h \simeq 1.1 \AA$. A recent pseudopotential calculation[92] also fou] and $(\mathrm{H}-\mathrm{B})$ as the two stable configurations. The (H-C) structure was found to be sligh stable than the (H-B) structure by an amount $\sim 0.03 \mathrm{eV} /$ dimer. Also in agreement with tl of Batra[90] they obtained $d(\mathrm{Na}-\mathrm{Si})=2.6$ and $2.8 \AA$ for the $\mathrm{Na}$ atoms at $\mathrm{H}$ and $\mathrm{C}$ sites, res] 
TABLE 4. Optimized atomic coordinates (in a.u.) and the residual forces (in units of $10^{-8} \mathrm{~N}$ ) on various atoms for $\mathrm{Na}-\mathrm{Si}(001)-(2 \times 1)$ at $\theta=2$ for the (H-B) and the (H-C) adsorption models[88]. These calculations were performed with $6.5 \mathrm{Ry}$ cutoff and $15 \mathrm{k}$-points in the BZ. No significant $\mathrm{y}$-forces $\left(F_{y}=0\right)$ were detected. Atomic coordinates for the other layers are those given in Table 2. (Reproduced from Ref. [88]).

\begin{tabular}{|cccccc|}
\hline $\mathrm{N}$ & $\mathrm{x}$ & $\mathrm{y}$ & $\mathrm{z}$ & $F_{x}$ & $F_{z}$ \\
\hline $\mathrm{Na}(\mathrm{H})$ & 0.0 & 3.63 & 2.38 & 0.0 & -0.014 \\
$\mathrm{Na}(\mathrm{B})$ & 7.26 & 0.0 & 0.67 & 0.0 & -0.013 \\
1 & -2.35 & 0.0 & -0.25 & -0.002 & -0.022 \\
$1^{\prime}$ & 2.35 & 0.0 & -0.25 & 0.002 & -0.022 \\
2 & -3.33 & 3.63 & -2.73 & 0.024 & 0.012 \\
$2^{\prime}$ & 3.33 & 3.63 & -2.73 & -0.024 & 0.012 \\
\hline $\mathrm{Na}(\mathrm{H})$ & 0.0 & 3.63 & 2.40 & 0.0 & -0.031 \\
$\mathrm{Na}(\mathrm{C})$ & 7.26 & 0.0 & 1.10 & 0.0 & -0.027 \\
1 & -2.50 & 0.0 & -0.25 & -0.012 & 0.016 \\
$1^{\prime}$ & 2.50 & 0.0 & -0.25 & 0.012 & 0.016 \\
2 & -3.33 & 3.63 & -2.73 & 0.004 & -0.008 \\
$2^{\prime}$ & 3.33 & 3.63 & -2.73 & -0.004 & -0.008 \\
\hline
\end{tabular}

\section{H. Third order $(\theta=2 / 3)$ structure}

A third order structure has been reported $[68,71]$ for $\mathrm{Na}, \mathrm{K}$ and $\mathrm{Cs}$ adsorption on $\mathrm{Si}(001)$ but no satisfactory model exists so far. Holtom and Gundry[68] observed the $2 \times 3$ structure at coverages $1 / 3 \leq \theta \leq 2 / 3$. They suggested that the low coverage $2 \times 3$ structure is due to Cs atoms at every fourth $\mathrm{H}$ site along the $y$-direction (see Fig. 14). Since the intensity of $(1 / 3,0)$ spot grew at the expense of $(2 / 3,0)$ spot with increasing coverage, they placed additional Cs atoms at the 
mid positions along the $y$-direction. This corresponds to placing the additional Cs atom at an adjacent short bridge site $\mathrm{D}$ (above the center of $\mathrm{Si}$ dimer) next to an $\mathrm{H}$ site. The unit cell is $2 \times 3$, with one $\mathrm{Cs}$ atom at $\mathrm{H}$ and another one at $\mathrm{D}$, for a coverage of $\theta=2 / 3$. If one looked at the overlayer alone (ignoring substrate altogether) it has the periodicity of $2 \times 1.5$. Such a unit cell does not give any primary third order spots. In practice because of the substrate the overall periodicity is $2 \times 3$ and hence $(1 / 3,0)$ spots shall be present but with reduced intensity. Thus with this mixed site occupancy model, Holtom and Gundry[68] successfully accounted for the LEED data. Batra[72] presented a slight variation of this model by noting that the $H$ and $B$ sites have nearly identical adsorption energies. Hence he suggested that the dimer bridge site $D$, invoked by Holtom and Gundry[68], ought to be replaced by B. Reduced intensity of the third order spots in Batra's model[88] follows from the fact that alkali atoms at $\mathrm{H}$ and $\mathrm{B}$ sites have different vertical heights above the surface.

Glander and Webb[71] have argued that none of the above mixed site models can account for their observations of the third order structure for $\mathrm{Na}-\mathrm{Si}(001)$. In particular, a drastic decrease of the intensity of the half-order beams led them to propose a major reconstruction of the Si substrate. This consisted of removing every third surface $\mathrm{Si}$ atom along the $x$-direction and allowing the second layer atoms beneath them to dimerize by appropriate $\pm \Delta y$ movements. The dimers at the surface are now separated by 3 along the $x$-direction while the second layer dimers are separated by 2 along the $y$-direction. Such a reconstruction clearly reduces the intensity of the half-order spots because the 2-periodicity is only due to dimers in the second Si layers. Furthermore, there is only one such dimer per $3 \times 2$ cell as compared to three dimers in the $2 \times 3$ cell involved in the mixed site models. Another nice feature of the reconstruction model is that it can continuously go to the $2 \times 1$ structure at $1 / 2 \mathrm{ML}$ by only filling the $\mathrm{H}$ sites. It is important to note that the proposed[71] reconstruction can not produce a $2 \times 3$ periodicity (only $3 \times 2$ ) and a mass transport is required. Furthermore, there is no other independent evidence yet for any major structural rearrangement in the substrate brought on due to the adsorption of alkali metals. The proposed reconstruction has eliminated the three surface $B$ sites and replaced them by a single buried B site. Since there are indications that the $\mathrm{B}$ site is also a preferred site (in addition to the $\mathrm{H}$ site) the reconstruction model may lead to an energy-deficit structure.

The competitive filling of the $B$ sites suggests that the model of the $2 \times 3$ structure requires some further discussion. At $\theta=1 / 3$, the third order structure can have adsorbates at either $\mathrm{H}$ or $\mathrm{B}$ sites as long as every fourth such site is occupied along the $y$-direction. With increasing coverage both sites must be occupied. An adsorbate in an $\mathrm{H}$ site blocks off four surface sites. Then the next 
alkali atom can adsorb in a $B$ site, but must skip the adjacent $2 \times 1$ cell. This gives rise to a $2 \times 3$ structure at $\theta=2 / 3(1 / 3 \mathrm{ML}$ coverage). The reduced intensity of $(1 / 3,0)$ and $(1 / 2,0)$ spots arises from puckering or different vertical adsorption heights at the two sites. The mixed site models have one additional constraint. They can produce a $2 \times 1$ structure (at $1 / 2 \mathrm{ML}$ ) as a function of increasing coverage only if there is sufficient energy available for diffusion. Remember in the mixed site model one occupies both $\mathrm{H}$ and $\mathrm{B}$ sites but for the $2 \times 1$ periodicity same symmetry sites must be occupied in all cells. For this to happen, alkali metal atoms must be able to move around. At monolayer coverage all $\mathrm{H}$ and $\mathrm{B}$ sites can be filled and one again recovers a $2 \times 1$ structure.

It might be argued that since the $B$ site is a viable adsorption site why not explain the entire coverage range with the $\mathrm{B}$ site occupancy. In this case the $2 \times 3$ structure shall arise by filling every fourth B site. But then the mechanism of skipping two B sites remains unclear. Also, this opens up the question of how $\mathrm{Si}(001)$ is activated to negative electron affinity state. Recall that the NEA has been explained[58,59,67] on the basis of the $\mathrm{H}$ site occupancy. In any case this is a subject that ought to be explored further.

\section{Nature of binding and electronic structure}

The nature of AM-semiconductor bond as a function of coverage is not fully understood yet. For AM on metals, Langmuir[94], Gurney[95], and more recently Lang[96] presented a model involving an ionic to metallic transition as a function of AM coverage. At low coverages, the AM atoms are largely ionized with the alkali valence $s$-level (broadened into a resonance) appearing above $E_{F}$. With increasing coverage, the depolarization effects shift the resonance downwards leading to a partial occupancy of the adatom s-level. At sufficiently high coverage the AM overlayer turns metallic with partially-filled[97,98] s-band. Some recent calculations[62] have established that AMmetal bond has some covalent component even at low coverages and it should be more precisely viewed as a polarized bond as opposed to strictly ionic bond.

For AM-semiconductor interactions the situation is even more complex $[5,66,85]$. Some of the publications $[69,99,100]$ suggested that the AM-metal picture can be literally taken over for AMsemiconductor interactions as well. This view is not universally accepted. It is argued $[66,84,88]$ that the presence of active dangling bonds on semiconductor surfaces either delays (to higher coverages compared to overlayer on metals) or suppresses the overlayer metallization altogether. Ciraci and Batra[66] carried out SCF-electronic structure calculations of clean and $\mathrm{K}$-adsorbed $\mathrm{Si}(001)-(2 \times 1)$ surface and found two bands in the gap (see Fig. 17) for both cases. For the clean surface these 


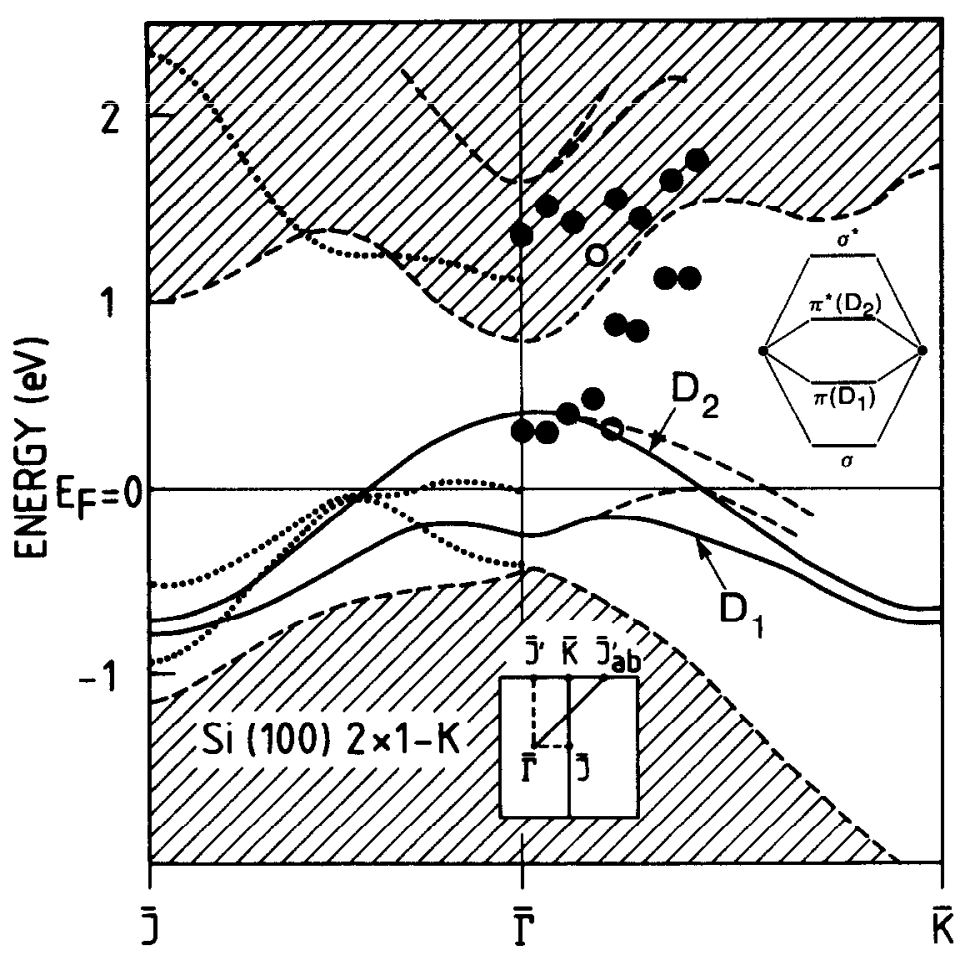

Fig. 17. Energy band structure of $\mathrm{K}$ adsorbed on the $\mathrm{H}$ site of the $\mathrm{Si}(001)-(2 \times 1)$ surface at $\theta=1$. The dangling bond bands $\left(D_{1}\right.$ and $\left.D_{2}\right)$ in the band gap and the resonance state $(R)$ in the conduction band are shown. The bands calculated by the tight-binding method (small dots) and ARUPS data (large dots) are obtained from Refs. [81] and [101], respectively.

were occupied and empty $\left(D_{1}\right.$ and $D_{2}$, respectively) dangling bond surface state bands. Upon Kadsorption at $\theta=1$ these bands are slightly modified with the Fermi level passing through $D_{2}$. The electrons from the alkali overlayer are accommodate in $D_{2}$ which is then partially occupied and cauces the metallization of the Si surface. Furthermore, they identified surface related resonance states in the region of conduction band where the plasmon dispersions are measured. These states are accessible by the excited electrons in the EELS sampling and are likely to be responsible for collective and individual excitations. The resonance state, $R$, hybridizes with different bulk conduction band states along the [100] direction. At $k=0$ this state is $1.9 \mathrm{eV}$ above the filled $D_{1}$ state. The individual excitation energy inferred from $D_{1}$ and $R$ is in fair agreement with the observed loss peak. The electronic energy structure calculated[66] for $\mathrm{K}$ adsorbed at the $\mathrm{H}$ site of the $\operatorname{Si}(001)-(2 \times 1)$ surface together with the ARUPS data[101] is presented in Fig. 17.

The nature of the gap states $D_{1}$ and $D_{2}$, and the resonance state $R$ was revealed[66] by comparing 

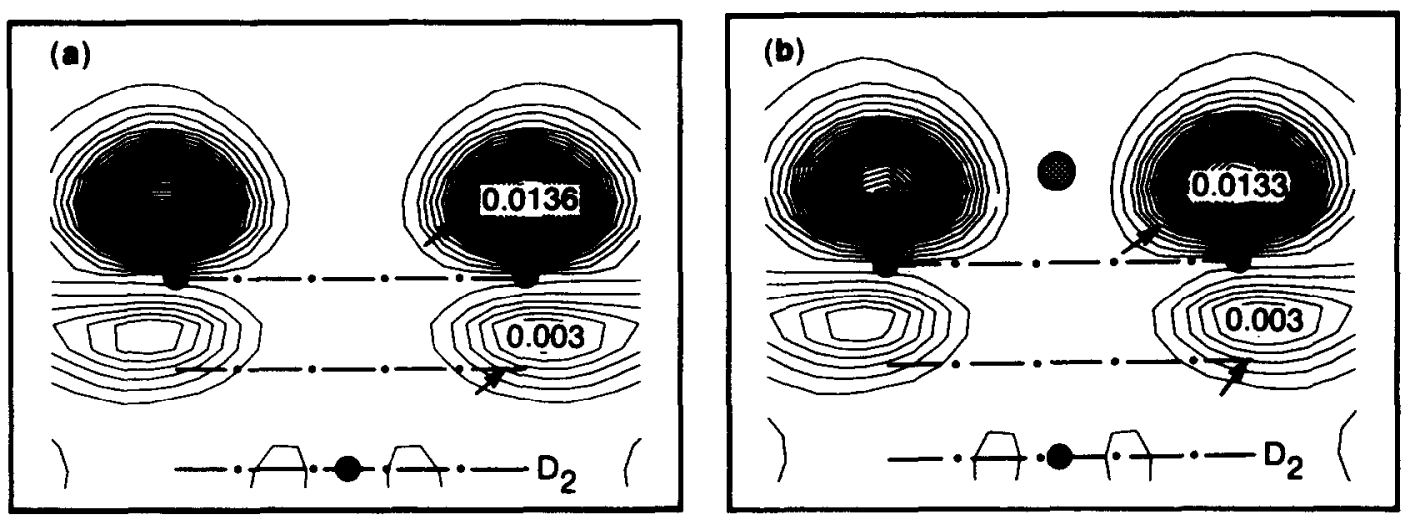

Fig. 18. Charge density contour plots for $D_{2}$ state calculated for $\vec{k}=(0.0,0.108,0.0)$ a.u..$^{-1}$ in a vertical plane passing through $K$ and two nearest surface Si atoms. (a) Clean, (b) $K$ adsorbed $\operatorname{Si}(001)-(2 \times 1)$ surface with $\theta=1$. (Reproduced from Ref. [66]).

their charge densities with those calculated for the clean $\mathrm{Si}(001)-(2 \times 1)$ surface. As shown in Fig. 18 it was found that the charge density of $D_{1}$ and $D_{2}$ emphasize the dangling bond origin with minor modifications from those of the clean $\mathrm{Si}(001)-(2 \times 1)$. In contrast to the gap states, the charge density of the resonance state in the conduction band $R$ was found to displays a significant alkali atom contribution. These results together with those obtained from the comparison made between the total charge densities with the unsupported K-monolayer and $\mathrm{K}$ adsorbed Si surface (see Fig. 19) are corroborating the above conclusion that the band determining the Fermi level at $\theta=1$ originates from the empty dangling bond surface state of the clean $\operatorname{Si}(001)-(2 \times 1)$. That is, the valence charge of the $K$ atom is donated to fill the surface dangling bond states, which in turn leads to the metallization of the Si-surface. In contrast, the alkali metal atoms absorbed on metal substrates regain charge at the saturation coverage. The presence of the active dangling bond states provides the crucial difference between a metal and a semiconductor substrate.

The electronic structure calculated by SCF-pseudopotential at $\theta=2$ is rather similar to that obtained at $\theta=1$. At $\theta=1$, a single alkali adsorbate atom per unit cell partly fills the upper dangling bond band leading to a metallic state. At $\theta=2$, the $2 \times 1$ unit cell has just enough electrons to fully occupy all dangling bond bands leading to a semiconducting surface. An important point is that the AM overlayers do not introduce any new bands around $E_{F}$ even at $\theta=2$. The reentrant behavior to the insulating state as a function of coverage thus follows naturally from SCF-pseudopotential calculations. The origin of the above metal-insulator transition is traced to the ionic interaction between the alkali metal and $\mathrm{Si}$, and to the presence of active surface states 


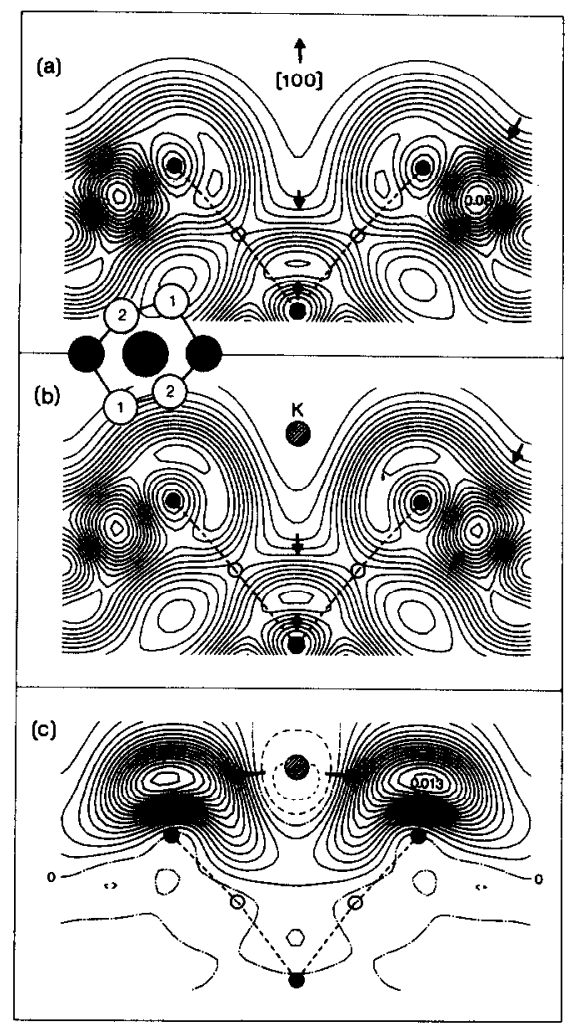

Fig. 19. Contour plots of the total density in a vertical plane passing through $\mathrm{K}$ and two nearest surface $\mathrm{Si}$ atoms. (a) Clean $\mathrm{Si}(001)-(2 \times 1)$, (b) K adsorbed $\mathrm{Si}(001)-(2 \times 1)$ with $d(\mathrm{~K}-\mathrm{Si})=4.9$ a.u. (c) Difference plot of $(\mathrm{a})$ and (b) showing regions of charge depletion (dashed lines) and charge accumulation (solid lines). Positions of the atoms in the plane and projected positions of atoms out of plane are shown by dots and circles, respectively.

on the Si surface.

A recent ARUPS study[58] for a single-domain $\mathrm{K}-\mathrm{Si}(001)-(2 \times 1)$ has been presented. Earlier experiments[101] were done on double-domain $\mathrm{Si}(001)-(2 \times 1)$ from which it is difficult to obtain dispersion of surface states in an unambiguous fashion. Single domain $\mathrm{Si}(001)-(2 \times 1)$ was obtained by growing epitaxial Si layers onto a $\mathrm{Si}$ wafer held at $500{ }^{\circ} \mathrm{C}$ and then annealing it at $1000{ }^{\circ} \mathrm{C}$. Potassium was then deposited on this substrate held at room temperature under a pressure of $\sim 6 \times 10^{-10}$ to obtain $\mathrm{K}$-saturated single domain $\mathrm{Si}(001)-(2 \times 1)$. Two filled surface state bands; $D_{1}$ and $D_{2}$, were indeed detected with ARUPS. In agreement with the above conclusion for $\theta=$ 2, no additional bands directly attributable to $K(4 s)$ were found and the surface was indeed semiconducting(see Fig. 17). 


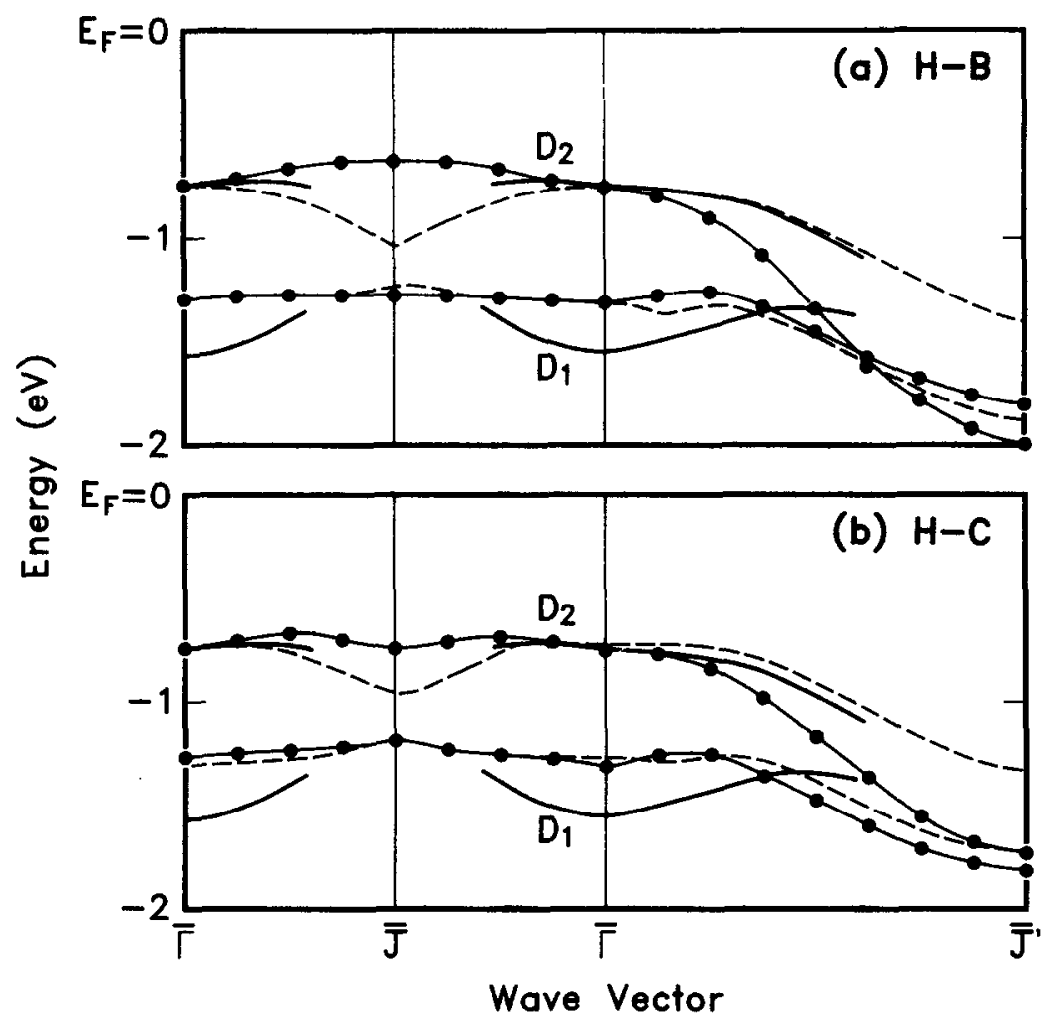

Fig. 20. Comparison of calculated electronic band structure for K-Si at $\theta=2$ with ARUPS data. Solid lines with dots are the results for $\mathrm{K}$ atoms at optimized positions above the $\mathrm{Si}$ surface. Dotted lines give dispersions computed for $\mathrm{K}$ placed at empirical heights. Results for (H-B) and (H-C) models are shown in panels (a) and (b), respectively. ARUPS data around $E_{F}$ on a single domain $\mathrm{Si}(001)-(2 \times 1)$ dosed to saturation with $\mathrm{K}$ is shown by solid lines in both panels. (Reproduced from Ref. [58]).

The surface bands are calculated by SCF-pseudopotential method and are shown in Fig. 20 for (H-B) as well as (H-C) sites occupancies by $\mathrm{K}$, corresponding to $\theta=2$. In view of some uncertainty in the computed $d(\mathrm{~K}-\mathrm{Si})$ values, we have also shown results by placing the dilayer at empirical height. The empirical $\mathrm{K}$ heights above the surface at $(\mathrm{H}-\mathrm{B})$ sites were obtained by fixing $d(\mathrm{~K}-\mathrm{Si})=$ $3.1 \AA$ for both $\mathrm{H}$ and B sites. This then led to $h_{1}=2.1 \AA$ (height above the H site) and $h_{2}=1.5 \AA$ (height above the $B$ site). These vertical heights were also used for calculations at (H-C) sites. The $\mathrm{K}$-dilayer is thus puckered by the same amount for (H-B) and (H-C) calculations. In all cases the energy of the $D_{2}$ band at the center of the Brillouin zone is set to coincide with the measured value. The shape, location and dispersion of the lower lying $D_{1}$ band is essentially independent of sites and vertical positions of $\mathrm{K}$-atoms. This band can be brought in better registry with the measured 
data by a $0.5 \mathrm{eV}$ shift downwards. Thus this band does not provide much help in deciding between (H-B) and (H-C) structures. The measured $D_{2}$ band is well described in terms of (H-B) sites for K's at empirical distances but only moderately less well by (H-C) occupancies. In view of the fact that one electron eigenvalues from Kohn-Sham equations are not precisely comparable to the measured bands in ARUPS, we can not really make a definitive choice. However, there is no doubt that both (H-B) and (H-C) lead to a semiconducting surface and support a picture of substrate metallization at coverages below saturation.

A well known problem is that there is no method which allows a rigorous calculation of the transfer of charge between two atoms. The existing models assign charges to the atoms under consideration according to the value of the integrated charge in the regions partitioned in an $a d$ hoc manner. For example, such a partitioning led Kasowski and Tsai[83] to conclude that charge, though small, is transferred from Si to $\mathrm{K}$ thus making alkali overlayer metallic. Assigning the charge from a plane $1.06 \AA$ above the surface $\mathrm{Si}$ atoms to infinity to the alkali overlayer they assigned 1.15 electron per $\mathrm{K}$ atom. It is clear that their scheme assigns some of the high charge density of the Si surface to the $\mathrm{K}$ overlayer. This can be seen from the SCF-charge density calculated for $d(\mathrm{~K}-\mathrm{Si})=6$ a.u. $(3.2 \AA)$ revealed from SEXAFS[80]. Plots of valence charge density difference i. e., $\rho[\mathrm{K}+\mathrm{Si}]-\rho[\mathrm{Si}]$ in Fig. 21 unambiguously show the filled dangling bond states as the origin of surface metallization, and not the metallic K chains. Since the lowest-value contour of clean Si surface in Fig. 20(b) is already larger than the maximum charge density of the unsupported $\mathrm{K}$ chain in Fig. 20(d), the scheme by Kasowski and Tsai[83] leads to an artificial transfer of charge from Si to less electronegative $\mathrm{K}$. As a matter of fact, the amount of charge between a plane from $1.06 \AA$ above the surface to infinity is larger than one electron even for the clean surface with half-filled dangling bond band.

Ling et al.[73] have carried out extensive cluster model calculations for $\mathrm{K}$-Si system within the framework of local-density total energy formalism using Hedin-Lundqvist form of exchangecorrelation potential. For the $\mathrm{H}$ site at $\theta=1$, they obtained a K-Si bond length of about $3.2 \AA$ in good agreement with experiment but longer than the value of $2.6 \AA$ calculated by Ciraci and Batra[66]. Furthermore, they found a strong (binding energy $\sim 3 \mathrm{eV}$ ) ionic bond ( $\Delta Q \sim 0.63 e$ from $\mathrm{K}$ to $\mathrm{Si}$ ). These findings tend to contradict the conclusion[102] that the alkali metal-Si bonding is weak and covalent.

A Mulliken population analysis, on the other hand, is useful but has its well known shortcoming due to the overlap of the atomic orbitals. In view of these uncertainties, Ciraci and Batra[66] did not perform any calculation for the transfer of charge with the plane wave basis set, but 


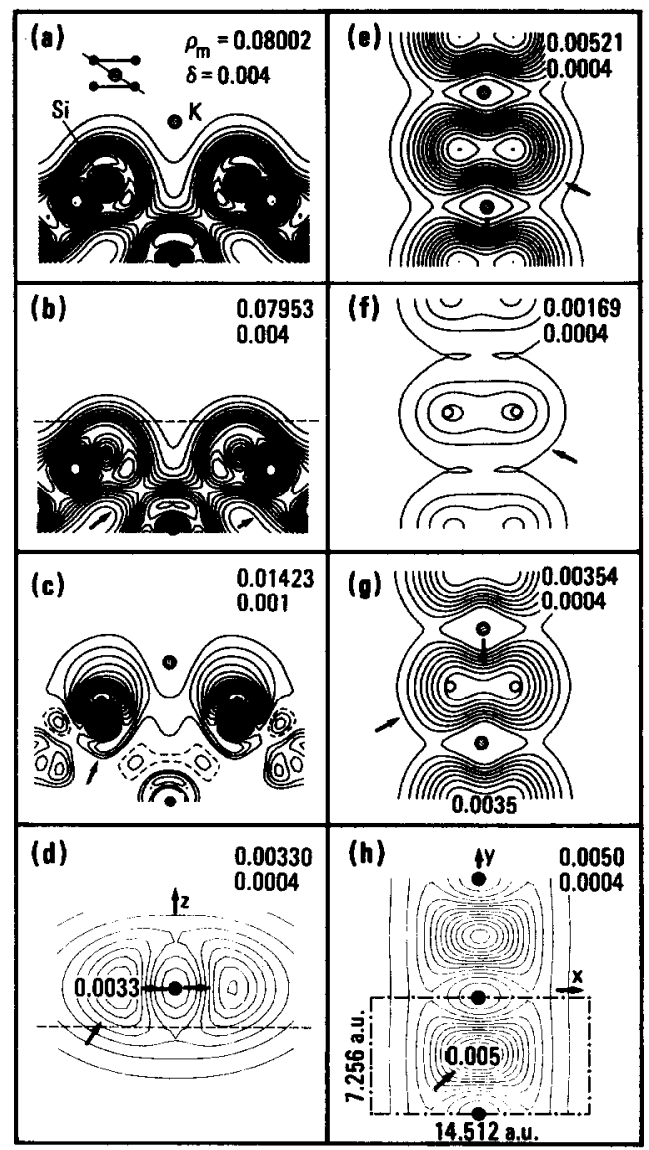

Fig. 21. Contours of total charge density. First column: in a vertical plane passing through $K$ and two nearest surface $\mathrm{Si}$ atoms. (a) $\mathrm{K}$ adsorbed $\mathrm{Si}(001)-(2 \times 1)$ with $d(\mathrm{~K}-\mathrm{Si})=6$ a.u. (b) clean $\mathrm{Si}(001)-(2 \times 1)$, (c) difference plot of (a) and (b), (d) unsupported, metallic $\mathrm{K}$ chain in registry with $\mathrm{K}$ on $\mathrm{Si}(001)-(2 \times 1)$. Second column: in the plane of $\mathrm{K}$ overlayer 4.2 a.u. above the silicon. (e)-(h) are in the above order. $\rho_{m}$, maximum value of charge, $\delta$, contour spacing. Dashed line in (b) indicates the lateral plane $1.06 \AA$ above the surface. (Reproduced from Ref.[83]).

deduced the ionic binding from the half-filled gap states $D_{2}$, which displays a dangling bond character. Later, SCF-Hartree-Fock calculations[84] for a small cluster and the charge transfer analysis with the method predicted $94 \%$ of the valence charge on the $\mathrm{K}$ atom is transferred to Si. SCF-pseudopotential calculations by Northrup[103] also assigned ionic binding for $\mathrm{Na}$ adsorbed on the $\operatorname{Si}(111)-(2 \times 1)$ surface. Perhaps owing to the arbitrariness indigenous to charge allocation published values on the estimates of charge transfer[92] $\Delta Q$ from $\mathrm{AM}$ to $\mathrm{Si}$ have varied from $0<\Delta Q<1$. 
The binding energy $E_{b}$ of the adsorbed $\mathrm{AM}$ overlayer at the $\mathrm{H}$ site, is obtained from Eq. (11 terms of the total energies of the AM-Si system, the bare substrate ( $\mathrm{Si}$ ) and the overlayer ( $t$ all calculated using slabs of same dimensions. The calculated binding energy for $\mathrm{K}$ adsorbec the $\mathrm{H}$ site of $\mathrm{Si}(001)-(2 \times 1)$ is $2.5 \mathrm{eV}$. Similarly, the binding energy is $E_{b}=2.5 \mathrm{eV}$ per $\mathrm{Na}$ in $2 \times 1$ cell for the $\mathrm{AB}$ model[87] of the substrate. The binding energy is reduced to $2.15 \mathrm{eV}$ if substrate coordinates optimized by Batra[88] are used. Completely optimized structure discu in Table 2 gives $E_{b}=2.23 \mathrm{eV}$. More stable $E_{b}$ in the $\mathrm{AB}$ model is primarily due to the hil reference substrate energy than the optimized substrate energy. These energies, however, sh، not be directly compared with the desorption energy of a single $\mathrm{Na}$ atom since the overlay desorbed as a whole. The computed values[88] have a deficit of an amount equal to the cohesio the overlayer. On the other hand, the local-density approximation overestimates bonding ener! Hence the absolute energies should be viewed with caution. But in agreement with ling et al. we conclude that the AM-Si bonding is strong.

The electronic structure of the $\mathrm{K}$ adsorbed $\mathrm{Si}(111)-(2 \times 1) \pi$-bonded surface and the bin derived thereof have character similar to that discussed for $\mathrm{Si}(001)-(2 \times 1)$ surface. Electr structure and total energy calculations[104] have shown that the alkali atoms are adsorbed at the center of the tilted hexagon formed surface ( $\pi$-chain) and subsurface $\mathrm{Si}$ atom. Accordir the topology of the surface charge density with two hollow sites in the superlattice cell offers possibility of accommodating $A M$ atoms up to $\theta=2$. The potassium atoms lying 2.8 a.u. at the center of the tilted hexagon $(h=1.8 \mathrm{a}$.u.) have the maximum binding energy $(2.8 \mathrm{eV} \mathrm{pe}$ atom). However, the average binding energy decreased to $2.5 \mathrm{eV}$ per $\mathrm{K}$ atom at $\theta=2$.

It is well known that the clean $\operatorname{Si}(111)-(2 \times 1) \pi$-bonded surface has two intrinsic surface $s$ bands. The $\pi$-band partially overlaps with the valence band and is completely filled. Whe: the $\pi^{*}$-band is empty and overlap with the conduction band along $\Gamma-J^{\prime}$ direction of the sur Brillouin zone. Consequently the clean $\pi$-bonded $\mathrm{Si}(111)$ surface is insulating. At the $\mathrm{K}$ coves corresponding to $\theta=1$, the Fermi level rises by $0.7 \mathrm{eV}$ with respect to the valence band edge $\pi^{*}$-band becomes partially occupied. As clarified by an extensive charge density analysis of clean and $\mathrm{K}$ covered surfaces[88], the overall form of the $\pi$ - and $\pi^{*}$-bands stays nearly intact $u$ $K$ adsorption, but the substrate surface undergoes a transition from an insulating to a met. state. Above the threshold coverage (estimated to be $\theta \simeq 0.9$ ) the extra charge is accommodi by the bulk conduction band, and the Fermi level stays almost pinned.

As of this writing it is not clear $[105,106]$ if the $\pi$-bonding reconstruction is lifted upon adsorption. There is some evidence[106] that this may well be a function of the specific a] 
metal under consideration.

A feature common to all the electronic energy structure obtained from the SCF-pseudopotential calculations with $\theta<2$ is that the valence electrons of the AM are donated to the dangling bond surface state bands. The overlayer metallization is suppressed, but the semiconductor surface is metallized. That the dangling bond states are maintained (perhaps with some degree of polarization at large $\theta$ ) is consistent with Heine's picture[7] described in section 2 , in which the semiconductor dangling bond states in the gap change into Breit-Wigner resonances. This is a situation rather different from that seen in the simple metal ( $\mathrm{Al}$ ) semiconductor interface in section 3, where M-M interaction wins over the M-S interaction leading to the overlayer metallization.

Finally, we would like to touch upon the Si $2 p$ core level shift. Earlier, it was argued[102] that the excess electrons transferred from the AM to $\mathrm{Si}$ would give rise to the core level shift. The absence of the shift was taken as an evidence for a predominantly covalent bond[80]. However, recent high-resolution core level $\mathrm{x}$-ray photoemission and photoabsorption studies[107] of $\mathrm{K}$ adsorption on the B-doped $\operatorname{Si}(111)-(\sqrt{3} \times \sqrt{3})$ surface showed a large core level shift $(\simeq-1.2 \mathrm{eV})$, for both B-1s and Si-2p states. This confirms the ionic picture proposed by Ciraci and Batra[66]. As for the $\operatorname{Si}(001)-(2 \times 1)$ surface it was pointed out that the average charge density of the $D_{2}$ states near the adsorbed $\mathrm{K}$ is comparable with the average density of the $\mathrm{K} 4 s$ state[66]. Hence, the charge of the $D_{2}$ state may provide an effective core screening preventing any observable Si $2 p$ core level shift. Most recently, the low-coverage site deduced from the STM studies has now been corrected[85]. The revised site, $\mathrm{Y}$ is a bridge site between two $\mathrm{Si}$ atoms belonging to different dimers along the dimer row[110] direction[85,108]. The total energy calculations for this new $Y$ site, discovered by STM have now been carried out[109]. Interestingly, the preliminary results indicate that $\mathrm{Si}$ atoms not directly bonded to the alkali metal buckle to yield the binding energy in the range of the binding energies calculated for $\mathrm{H}$ and $\mathrm{B}$ site. Nevertheless, energetically favorable adsorption sites at different coverages and the core shift are subjects which are being pursued actively[108-110] at the time of this writing. It is perhaps too early to close the book on this subject.

\section{References}

1. W. Schottky, Naturwissenschaffen 26, 843, 1938); Phys. Z. 41, 570 (1940).

2. S. M. Sze, Semiconductor Devices: Physics and Technology, John Wiley, (1985). 
3. R. H. Williams, Contemp. Phys. 23, 329 (1982); E. H. Rhoderick and R. H. Williams, Metal-Semiconductor Contacts, Oxford Univ. Press, New York, (1988).

4. L. J. Brillson, Surf. Sci. Rep. 2, 123 (1982).

5. For a collection of recent studies, see, I. P. Batra, ed., Metallization and Metal-Semiconductor Interfaces, Plenum, New York, (1989).

6. J. Bardeen, Phys. Rev. 71, 717 (1947).

7. V. Heine, Phys. Rev. 138, A1689 (1965).

8. E. Louis, F. Yndurain, and F. Flores, Phys. Rev. B13, 4408 (1976).

9. J. Tersoff, Phys. Rev. Lett. 52, 465 (1984); Phys. Rev. B30, 4874 (1984).

10. W. E. Spicer, I. Lindau, P. R. Skeath, C. Y. Su, and P. W. Chye, Phys. Rev. Lett. 44, 420 (1980); W. E. Spicer, P. W. Chye, P. R. Skeath, C. Y. Su, and W. G. Spitzer, J. Vac. Sci. Technol. 16, 1422 (1979).

11. I. Lindau and T. Kendelewicz, Crit. Rev. Solid State Mater. Sci. 13, 27 (1986).

12. W. E. Spicer, Z. Liliental-Weber, E. R. Weber, N. Newman, T. Kendelewicz, R. Cao, C. McCants, K. Miyano, P. H. Mahowald, and I. Lindau, J. Vac. Sci. Technol. B6, 1245 (1988).

13. R. Ludeke, G. Jezequel, and A. Taleb-Ibrahimi, Phys. Rev. Lett. 61, 601 (198).

14. R. Ludeke, Phys. Rev. B40, 1947 (1989); in Metallization and Metal-Semiconductor Interfaces, I. P. Batra, ed., pp. 39-54, Plenum, New York, (1989).

15. C. M. Aldao, S. G. Anderson, C. Capasso, G. D. Waddill, I. M. Vitomirov, and J. H. Weaver, Phys. Rev. B39, 12977 (1989).

16. C. Mailhiot and C. B. Duke, Phys. Rev. B33, 118 (1986).

17. J. L. Freeouf and J. M. Woodall, App. Phys. Lett. 39, 727 (1981).

18. I. Lefebvre, M. Lannoo, C. Priester, G. Allan, and C. Delerue, Phys. Rev. B36, 1336 (1987); M. Lannoo, C. Priester, G. Allan, I. Lefebvre, and C. Delerue, in Metallization and MetalSemiconductor Interfaces, I. P. Batra, ed., pp. 259-268, Plenum, New York, (1989).

19. A. M. Cowley and S. M. Sze, App. Phys. Lett. 36, 3212 (1965).

20. S. Kurtin, T. C. McGill, and C. A. Mead, Phys. Rev. Lett. 22, 1433 (1969).

21. J. C. Phillips, Solid State Commun. 12, 861 (1973). 
22. J. C. Inkson, J. Vac. Sci. Technol. 11, 943 (1974).

23. J. M. Andrews and J. C. Phillips, Phys. Rev. Lett. 35, 56 (1975); L. J. Brillson, Phys. Rev. Lett. 40, 260 (1978).

24. M. Schlüter, Phys. Rev. B17, 5044 (1978).

25. W. Mönch, J. Vac. Sci. Technol. B6, 1270 (1988); in Metallization and Metal-Semiconductor Interfaces, I. P. Batra, ed., pp. 11-38, Plenum, New York, (1989).

26. E. J. Mele and J. D. Joannopoulos, Phys. Rev. B17, 1528 (1978).

27. Y. Chang, Y. Hwu, J. Hansen, F. Zanini, and G. Margaritondo, Phys. Rev. Lett. 63, 1845 (1989).

28. L. J. Brillson and P. Chiaradia, in Metallization and Metal-Semiconductor Interfaces, I. P. Batra, ed., pp. 91-110, Plenum, New York, (1989).

29. S. G. Louie and M. L. Cohen, Phys. Rev. B13, 2461 (1976).

30. S. G. Louie, J. R. Chelikowsky, and M. L. Cohen, Phys. Rev. B15, 2154 (1977).

31. C. Tejedor, F. Flores, and E. Louis, J. Phys. C10, 2163 (1977).

32. F. Guinea, J. Sánchez-Dehesa, and F. Flores, J. Phys. C16, 6499 (1983); J. Sánchez-Dehesa, F. Flores, and F. Guinea, J. Phys. C17, 2039 (1984).

33. J. Tersoff, Phys. Rev. B32, 6968 (1985).

34. W. A. Harrison, J. Vac. Sci. Technol. B3, 1231 (1985).

35. W. A. Harrison and J. Tersoff, J. Vac. Sci. Technol. B4, 1068 (1986).

36. J. Tersoff and W. A. Harrison, Phys. Rev. Lett. 58, 2367 (1987).

37. W. E. Spicer, R. Cao, K. Miyano, C. McCants, T. T. Chiang, C. J. Spindt, N. Newman, T. Kendelewicz, I. Lindau, E. Weber, and Z. Liliental-Weber, in Metallization and MetalSemiconductor Interfaces, I. P. Batra, ed., pp. 139-161, Plenum, New York, (1989).

38. O. F. Sankey, R. E. Allen, and J. D. Dow, J. Vac. Sci. Technol. B3, 491 (1984).

39. R. E. Allen and J. D. Dow, Phys. Rev. B25, 1423 (1982); O. F. Sankey, R. E. Allen, S. F. Ren, and J. D. Dow, J. Vac. Sci. Technol. B3, 1162 (1984).

40. M. S. Daw and D. L. Smith, Phys. Rev. B20, 5150 (1979).

41. A. Zur, T. C. McGill, and D. L. Smith, Phys. Rev. B28, 2060 (1983). 
42. S. Doniach, K. K. Chin, I. Lindau, and W. E. Spicer, Phys. Rev. Lett. 58, 591 (1987

43. S. Ciraci and I. P. Batra, Solid State Commun. 51, 43 (1984).

44. I. P. Batra and S. Ciraci, Phys. Rev. B29, 6419 (1984); J. Vac. Sci. Technol. B3, 427

45. I. P. Batra and S. Ciraci, Phys. Rev. B33, 4312 (1986).

46. K. Stiles and A. Kahn, Phys. Rev. Lett. 60, 440 (1988); A. Kahn, K. Stiles, D. Mac Horng, K. Young, J. McKinley, D. G. Kilday, and G. Margaritondo, in Metallizati Metal-Semiconductor Interfaces, I. P. Batra, ed., pp. 163-178, Plenum, New York, (1

47. P. Masri, Phys. Rev. Lett. 64, 208 (1990).

48. I. P. Batra, Phys. Rev. B29, 7108 (1984).

49. Shang-Lin Weng, A. Y. Cho, W. C. Marra, and P. Eisenberger, Solid State Comm $843(1980)$.

50. S. Ciraci, A. Baratoff, and I. P. Batra, Phys. Rev. B43, in press (1991).

51. S. B. Zhang, M. L. Cohen, and S. G. Louie, Phys. Rev. B34, 768 (1986).

52. G. P. Das, P. Blöchl, O. K. Andersen, N. E. Christensen, and O. Gunı Phys. Rev. Lett. 63, 1168 (1989).

53. A. U. Mac Rae, K. Muller, J. J. Lander, J. Morrison, and J. C. Phillips, Phys. Rev. Lr $1048(1969)$.

54. R. U. Martinelli, App. Phys. Lett. 16, 261 (1970).

55. R. U. Martinelli and D. G. Fisher, Proc. IEEE 62, 1339 (1974).

56. B. Hellsing, Phys. Rev. B40, 3855 (1989).

57. H. Tochihara, Surf. Sci. 126, 523 (1983); T. Aruga, H. Tochihara, Y. I Phys. Rev. Lett. 53, 372 (1984).

58. Y. Enta, T. Kinoshita, S. Suzuki, and S. Kono, Phys. Rev. B36, 9801 Phys. Rev. B39, 1125 (1989); Y. Enta, S. Suzuki, S. Kono, and T. Sak Phys. Rev. B39, 5524 (1989).

59. T. Abukawa and S. Kono, Phys. Rev. B37, 9097 (1988).

60. H. Tochihara, Surf. Sci. (to be published).

61. R. L. Gerlach and T. N. Rhodin, Surf. Sci. 19, 403 (1970). 
62. E. Wimmer, A. J. Freeman, J. R. Hiskes, and A. M. Karo, Phys. Rev. B28, 3074 (1983); H. Ishida and K. Terakura, Phys. Rev. B38, 5752 (1988).

63. H. P. Bonzel, Surf. Sci. Report 8, 1 (1988).

64. W. Mönch, Phys. Rev. B37, 7129 (1988); also see Ref. [25].

65. T. M. Wong, D. Heskett, N. J. Dinardo, and E. W. Plummer, Surf. Sci. 208, L1 (1989).

66. S. Ciraci and I. P. Batra, Phys. Rev. Lett. 56, 877 (1986); Phys. Rev. B37, 2955 (1988); in Metallization and Metal-Semiconductor Interfaces, I. P. Batra, ed., pp. 335-350, Plenum, New York, (1989).

67. J. D. Levine, Surf. Sci. 34, 90 (1973).

68. R. Holtom and P. M. Gundry, Surf. Sci. 63, 263 (1977).

69. E. M. Oellig and R. Miranda, Surf. Sci. 177, L947 (1986).

70. C. H. Wei, H. Huang, S. Y. Tong, G. S. Glander, and M. B. Webb, Phys. Rev. B42, 11284 (1990).

71. G. S. Glander and M. B. Webb, Surf. Sci. 222, 64 (1989); Surf. Sci. 224, 60 (1989).

72. I. P. Batra, Phys. Rev. B39, 3919 (1989).

73. Y. Ling, A. J. Freeman, and B. Delley, Phys. Rev. B39, 10144 (1989).

74. R. Ramirez, Phys. Rev. B40, 3962 (1989); C. K. Ong, J. Phys: Condens. Matter 2, 6731 (1989).

75. B. Goldstein, Surf. Sci. 35,227 (1973).

76. J. A. Stroscio, R. M. Feenstra, D. M. Newns, and A. P. Fein, J. Vac. Sci. Technol. A6, 499 (1988).

77. Y. Hasegawa, I. Kamiya, T. Hashizume, T. Sakurai, H. Tochihara, M. Kubota, and Y. Murata, Phys. Rev. B41, 9688 (1990).

78. P. Badt, A. Brodde, St. Tosch, and H. Neddermeyer, J. Vac. Sci. Technol. A8, 251 (1990).

79. P. Pervan, E. G. Michel, G. R. Castro, R. Miranda, and K. Wandelt J. Vac. Sci. Technol. A7, 1885 (1989); also see Ref. [5].

80. T. Kendelewicz, P. Soukiassian, R. S. List, J. C. Woicik, P. Pianetta, I. Lindau, and W. E. Spicer, Phys. Rev. B37, 7115 (1988). 
81. M. Tsukada, H. Ishida, and N. Shima, Phys. Rev. Lett. 53, 376 (1984); H. Ishida, N Shima, and M. Tsukada, Phys. Rev. B32, 6236 (1985).

82. M. Tsukada, N. Shima, Z. Zhu, H. Ishida, and K. Terakura, in Metallization and MetalSemiconductor Interfaces, I. P. Batra, ed., pp. 351-366, Plenum, New York, (1989).

83. R. V. Kasowski and M. H. Tsai, Phys. Rev. Lett. 60, 546 (1988)., See response to this comment by, S. Ciraci and I. P. Batra, ibid., 60, 547, 1988.

84. P. S. Bagus and I. P. Batra, Surf. Sci. 206, L895 (1988); I. P. Batra and P. S. Bagus, J. Vac. Sci. Technol. A6, 600 (1988).

85. For some recent reviews, see, I. P. Batra, Progress in Surf. Sci. 25, 175 (1987); J. Chimie Phys. 86, 689 (1989); H. P. Bonzel, A. M. Bradshaw, and G. Ertl, eds., Physics and Chemistry of Alkali Metal Adsorption, Elsevier, New York, (1989).

86. M. T. Yin and M. L. Cohen, Phys. Rev. B24, 2303 (1981); D. J. Chadi, Phys. Rev. Lett. 43, 43 (1979); K. C. Pandey, in Proc. 17th Intl. Conf. Phys. Semiconductor Conf., D. J. Chadi and W. A. Harrison, eds., p. 55, Springer, Berlin, (1984).

87. F. F. Abraham and I. P. Batra, Surf. Sci. 163, L752 (1985); I. P. Batra, Phys. Rev. B41, 5048 (1990);

88. I. P. Batra, J. Vac. Sci. Technol. A8, 3425 (1990); Phys. Rev. B, (to be published); for Peierls' distortions see, I. P. Batra, Phys. Rev. B42, 9162 (1990).

89. T. Aruga, H. Tochihara, and Y. Murata, Phys. Rev. Lett. 53, 372 (1984).

90. I. P. Batra, Phys. Rev. Lett. 63, 1704 (1989).

91. J. Nogami, S. Park, and C. F. Quate, App. Phys. Lett. 53, 2086 (1988); B. Bourguignon, K. L. Carleton, and S. R. Leone, Surf. Sci. 204, 455 (1988).

92. H. Ishida and K. Terakura, Phys. Rev. B40, 11519 (1989); K. Kobayashi, K. Terakura, and S. Blügel, (to be published).

93. A. J. Freeman, S. Tang, S. H. Chou, Y. Ling, and B. Delley, (to be published).

94. I. Langmuir, Phys. Rev. 23, 112 (1924).

95. R. W. Gurney, Phys. Rev. 47, 479 (1935).

96. N. D. Lang, Phys. Rev. B4, 4234 (1971).

97. J. P. Muscat and I. P. Batra, Phys. Rev. B34, 2889 (1986); P. A. Serena, J. M. Soler, N. Garcia, and I. P. Batra, Phys. Rev. B36, 3452 (1987). 
98. B. Woratschek, W. Sesselman, J. Küppers, G. Ertl, and H. Haberland, Phys. Rev. Lett. 55, 1231 (1985).

99. H. Tochihara, M. Kubota, M. Miyao, and Y. Murata, Surf. Sci. 158, 497 (1985); Y. Murata, H. Tochihara, and M. Kubota, in Metallization and Metal-Semiconductor Interfaces, I. P. Batra, ed., pp. 367-380, Plenum, New York, (1989).

100. E. M. Oellig, E. G. Michel, M. C. Asensio, R. Miranda, J. C. Duran, A. Munoz, and F. Flores, Europhys. Lett. 5, 727 (1988); J. E. Ortega, E. M. Oellig, J. Ferron, and R. Miranda, Phys. Rev. B36, 6213 (1987).

101. I. P. Batra, J. M. Nicholls, and B. Reihl, J. Vac. Sci. Technol. A5, 898 (1987).

102. P. Soukiassian, M. H. Bakshi, Z. Hurych, and T. M. Gentle, Surf. Sci. 221, L759 (1989).

103. J. E. Northrup, J. Vac. Sci. Technol. A4, 1404 (1986).

104. S. Ciraci and I. P. Batra, Phys. Rev. Lett. 58, 1982 (1987).

105. I. P. Batra and S. Ciraci, Phys. Rev. B37, 8432 (1988).

106. B. Reihl and K. O. Magnusson, Phys. Rev. B42, 11839 (1990).

107. Y. Ma, Phys. Rev. Lett. 65, 2173 (1990).

108. D. M. Riffe, G. K. Wertheim, J. E. Rowe, and P. H. Citrin, (to be published).

109. I. P. Batra and S. Ciraci, (to be published).

110. See for example, Symposium on Alkali Metal-Surface Interactions, Bull. Am. Phys. Soc. 36, 952 (1991). 